\title{
Algumas Caracterizações dos Operadores Compactos entre determinados Espaços de Banach
}

\author{
Fernanda Cardoso Estevam
}

DISSERTAÇÃO APRESENTADA AO

INSTITUTO DE MATEMÁTICA E ESTATÍSTICA DA

UNIVERSIDADE DE SÃO PAULO

PARA OBTENÇÃO DO GRAU DE MESTRE EM MATEMÁTICA

Área de concentração: Análise

Orientadora: Profa. Dra. Mary Lilian Lourenço

Durante o programa de mestrado, a autora obteve

apoio financeiro do $\mathrm{CNPq}$

São Paulo, 11 de janeiro de 2005. 



\title{
Algumas Caracterizações dos Operadores Compactos entre determinados Espaços de Banach
}

\author{
Este exemplar corresponde à redação final \\ da dissertação devidamente corrigida e \\ defendida por Fernanda Cardoso Estevam \\ e aprovada pela comissão julgadora.
}

São Paulo, 11 janeiro de de 2005.

Banca examinadora:

- Profa. Dra. Mary Lilian Lourenço (orientadora) - IME-USP

- Prof. Dr. Humberto Daniel Carrión Villaroel - IME-USP

- Prof. Dr. Mario Carvalho de Matos - Unicamp 

A Marcus Harada Penna, por seu amor incondicional 



\section{Agradecimentos}

Agradeço à minha orientadora Profa. Dra. Mary Lilian por sua orientação, paciência, incentivo, confiança, suas sugestões, seus conselhos e pelo seu carinho.

A todos os professores do IME-USP que contribuíram para minha formação. Aos meus amigos e colegas do IME-USP, em especial à Neusa Nogas Tocha.

A Marcus Harada Penna pelo incentivo, apoio e grande ajuda a mim dedicados durante o programa de mestrado e durante a elaboração deste texto.

Ao $\mathrm{CNPq}$ pelo apoio financeiro recebido durante o programa de mestrado. 



\section{Resumo}

O objetivo desta dissertação é estudar algumas caracterizações dos operadores compactos entre espaços de Banach. Para isso estudamos um resultado onde o espaço de Banach $\ell_{1}$ é o único espaço de Banach $E$ com uma base normalizada $\left(u_{n}\right)_{n}$ tal que cada operador linear compacto $T: F \rightarrow E$ tem uma representação da forma $T x=\sum g_{n}(x) u_{n}$, para cada $x \in F$, com $F$ um espaço de Banach e $\sum g_{n}$ uma série $\omega^{*}$ incondicionalmente convergente no dual topológico $F^{\prime}$ de $F$. Também estudamos algumas caracterizações dos espaços de Banach $F$ para os quais todos os operadores lineares contínuos de $C(\Omega)$ em $F$ sejam compactos, com $\Omega$ um espaço de Hausdorff compacto. Os resultados apresentados aqui encontram-se nos textos científicos [2] e [21].

\section{Abstract}

The main purpose of this work is to study some characterizations of compact operators in Banach spaces. In this way, we have studied a result where the Banach space $\ell_{1}$ is the only Banach space $E$ with a normalized base $\left(u_{n}\right)_{n}$ such that every compact linear operator $T: F \rightarrow E$ has a representation of the form $T x=\sum g_{n}(x) u_{n}$, for each $x \in F$, where $F$ is a Banach space and $\sum g_{n}$ is a $\omega^{*}$ unconditionally convergent series in the dual $F^{\prime}$ of $F$. We also have studied some characterizations on a Banach space $F$ for which all continuous linear operators from $C(\Omega)$ into $F$ are compact, where $\Omega$ is a compact Hausdorff space. The results studied here were presented on the papers [2] and [21]. 


\section{Sumário}

Introdução 3

1 Definições e resultados preliminares $\quad 7$

1.1 Conceitos Básicos . . . . . . . . . . . . . . . . . . . 8

1.2 Topologia fraca em espaços de Banach . . . . . . . . . . . . . . 18

1.3 Séries e Bases de Schauder . . . . . . . . . . . . . . . . . 21

1.4 Operadores compactos . . . . . . . . . . . . . . . 35

$1.5 \mathrm{O}$ espaço $K\left(\ell_{2}, \ell_{2}\right) \ldots \ldots \ldots \ldots \ldots \ldots$

2 Uma caracterização de operadores compactos atráves de $\ell_{1} \quad 49$

3 Espaços de Banach $F$ para os quais $L(C(\Omega), F)=K(C(\Omega), F) \quad 69$

3.1 Caracterizações para Espaços Compactos Dispersos . . . . . . . . . . . . . 69

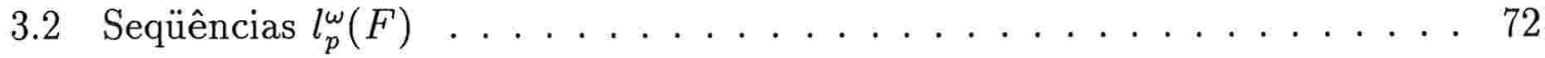

3.3 Caracterizações para Espaços Compactos Não Dispersos . . . . . . . . . . 80

3.4 Fatoração . . . . . . . . . . . . . . . . . . 83

Referências Bibliográficas $\quad 85$ 


\section{Introdução}

Nesta dissertação temos por objetivo estudar algumas caracterizações dos operadores compactos entre determinados espaços de Banach. Os resultados apresentados neste trabalho encontram-se nos textos científicos Ansari [2] e Randtke [21].

No capítulo 1 apresentamos as notações, definições e resultados de Análise Funcional e Geometria de espaços de Banach que utilizamos no decorrer da dissertação. Isto é, na primeira seção apresentamos conceitos básicos e na segunda seção apresentamos a definição e algumas propriedades de topologia fraca. Na terceira seção apresentamos alguns resultados sobre séries e bases de Schauder. Na quarta seção apresentamos alguns resultados importantes dos operadores compactos e fracamente compactos como os teoremas de Schauder e de Gantmacher. Encontram-se na quinta seção alguns resultados do espaço de todos os operadores compactos entre o espaço $\ell_{2}$; esse é um exemplo de espaço de operadores compactos entre espaços de Banach que contém um subespaço isométrico a $c_{0}$, que não é reflexivo e não é complementado em $L\left(\ell_{2}, \ell_{2}\right)$.

No capítulo 2 apresentamos algumas caracterizações dos espaços de Banach $E$ para os quais todos os operadores compactos de um espaço de Banach $F$ em $E$ admitam uma particular representação em séries. Mostramos que $\ell_{1}$ é o único espaço de Banach $E$ com base normalizada $\left(u_{n}\right)_{n}$ tal que todo operador linear compacto $T: F \rightarrow E$ de um espaço de Banach $F$ em $E$ tem uma representação da forma $T x=\sum g_{n}(x) u_{n} \operatorname{com} \sum g_{n}$ uma série $\omega^{*}$ incondicionalmente convergente no dual $F^{\prime}$ de $F$.

No capítulo 3 estudamos algumas caracterizações dos espaços de Banach $F$ para os quais todos os operadores contínuos de $C(\Omega)$ em $F$ sejam compactos, com $\Omega$ um espaço de Hausdorff compacto. Estas caracterizações dependem do espaço $\Omega$ ser disperso ou não disperso. Na primeira seção apresentamos os resultados para $\Omega$ disperso. A segunda seção caracteriza em termos de seqüencias $l_{p}^{\omega}(F)$, os espaços de Banach $F$ para os quais todos os 
operadores lineares contínuos de $E$ em $F$ são compactos, com $E=c_{0}$ ou $\ell_{p}(1 \leq p<\infty)$. As caracterizações para $\Omega$ não disperso estão na terceira seção. Terminamos esse capítulo apresentando na quarta seção alguns resultados que relacionam o espaço $K(C(\Omega), C(\Omega))$, de todos os operadores compactos entre $C(\Omega)$, com o espaço $\Phi_{c_{0}}(C(\Omega), C(\Omega))$ de todos os operadores contínuos entre $C(\Omega)$ que admitem uma fatoração através de $c_{0}$, com $\Omega$ qualquer espaço de Hausdorff compacto (disperso ou não disperso). 


\section{Capítulo 1}

\section{Definições e resultados preliminares}

Neste capítulo apresentaremos as notações, definições e alguns resultados de Análise Funcional e Geometria de espaços de Banach que serão utilizados no decorrer desta dissertação.

\section{Notações}

A menos de menção explícita do contrário, vamos utilizar a seguinte notação: IN denotará o conjunto dos números naturais;

$\mathbb{R}$, o conjunto dos números reais;

$\mathscr{C}$, o conjunto dos números complexos;

$\mathbb{I K}$, o corpo $\mathbb{R}$ ou $\mathbb{C}$;

$E$ e $F$, espaços de Banach sobre $\mathbb{K}$;

$L(E, F)$, o espaço de Banach dos operadores lineares contínuos de $E$ em $F$,

com a norma usual $\|T\|=\sup _{\|x\| \leq 1}\|T(x)\|$;

$E^{\prime}$, o espaço dual de $E$, o espaço $L(E, \mathbb{K})$;

$\ell_{1}, c_{0}, \ell_{\infty}$, os usuais espaços de Banach das seqüências absolutamente somáveis,

seqüencias convergentes a zero e seqüências limitadas, respectivamente;

$\Omega$, um espaço de Hausdorff compacto,

e $C(\Omega)$, o espaço de Banach de todas as funções contínuas $f: \Omega \rightarrow \mathbb{K}$, com a norma usual $\|f\|_{\infty}=\sup \{|f(x)|: x \in \Omega\}$;

$\left[x_{n}: n \in \mathbb{N}\right]$ denotará o subespaço gerado por $\left(x_{n}\right)_{n}$; 
$\overline{\left[x_{n}: n \in \mathbb{I N}\right]}$, o subespaço fechado gerado por $\left(x_{n}\right)_{n}$;

$B_{E}$, a bola unitária fechada de $E$;

$S_{E}$, a esfera unitária de $E$;

$x_{n} \rightarrow x$ denotará que a seqüência $\left(x_{n}\right)_{n}$ converge para $x$;

$x_{n} \not \nrightarrow x$, que a seqüência $\left(x_{n}\right)_{n}$ não converge para $x$;

$x_{n} \stackrel{\omega}{\rightarrow} x$, que a seqüência $\left(x_{n}\right)_{n}$ converge fracamente para $x$.

Usaremos a palavra espaço tanto do ponto de vista topológico como de espaço vetorial.

\subsection{Conceitos Básicos}

Começamos apresentando nesta seção definições e resultados básicos de espaços de Banach.

Definição 1.1.1. Dados $E$ e $F$ espaços normados, considere $L(E, F)$ como sendo o conjunto dos operadores lineares contínuos de $E$ em $F$.

As operações usuais de adição e multiplicação por escalar fazem de $L(E, F)$ um espaço vetorial, e a aplicação $\|\cdot\|$ de $L(E, F)$ em $\mathbb{R}_{+}$dada por $\|T\|=\sup _{\|x\| \leq 1}\|T(x)\|$ constitui uma norma em $L(E, F)$. Se $F$ é um espaço de Banach, entẫo $L(E, F)$ também é um espaço de Banach.

Teorema 1.1.2 (Teorema de Hahn-Banach). Sejam $X$ um espaço normado, $M$ um subespaço de $X$ e $f: M \rightarrow \mathbb{K}$ um funcional linear contínuo. Então $f$ pode ser estendido a um funcional linear contínuo $F: X \rightarrow \mathbb{K}$ tal que $\|F\|=\|f\|$.

Demonstração: Ver por exemplo, Megginson [16], pág. 75, teorema 1.9.6.

Como conseqüência do Teorema de Hahn-Banach temos o seguinte corolário.

Corolário 1.1.3. Sejam $X$ um espaço normado e $x_{0} \in X$. Então

$$
\left\|x_{0}\right\|=\sup \left\{\left|x^{\prime}\left(x_{0}\right)\right|: x^{\prime} \in B_{X^{\prime}}\right\} .
$$


Demonstração: Se $x_{0}=0$, então o resultado é imediato. Se $x_{0} \neq 0$, para $x^{\prime} \in B_{X}$, $\left|x^{\prime}\left(x_{0}\right)\right| \leq\left\|x^{\prime}\right\|\left\|x_{0}\right\| \leq\left\|x_{0}\right\| . \operatorname{Logo} \sup \left\{\left|x^{\prime}\left(x_{0}\right)\right|: x^{\prime} \in B_{X^{\prime}}\right\} \leq\left\|x_{0}\right\|$.

Sejam $M=\left[x_{0}\right]$ e $f: M \rightarrow \mathbb{K}$ um funcional linear definido por $f\left(\lambda x_{0}\right)=\lambda\left\|x_{0}\right\|$, temos que $\|f\|=1$. Pelo teorema de Hahn-Banach (teorema 1.1.2), $f$ pode ser estendido para um funcional linear contínuo $F: X \rightarrow \mathbb{K}$ tal que $\|F\|=\|f\|$. Assim temos que $\|F\|=1$ e $F\left(x_{0}\right)=\left\|x_{0}\right\|$. Conseqüentemente $\left\|x_{0}\right\|=\left|F\left(x_{0}\right)\right| \leq \sup \left\{\left|x^{\prime}\left(x_{0}\right)\right|:\right.$ $\left.x^{\prime} \in B_{X^{\prime}}\right\}$. Assim $\left\|x_{0}\right\|=\sup \left\{\left|x^{\prime}\left(x_{0}\right)\right|: x^{\prime} \in B_{X^{\prime}}\right\}$.

Definições 1.1.4. Sejam E e F espaços de Banach sobre $\mathbb{K}$.

1. Um operador $T \in L(E, F)$ é dito um isomorfismo de $E$ sobre $F$ se $T$ é uma bijeção $e$ $T^{-1} \in L(F, E)$. Os espaços $E$ e $F$ são ditos isomorfos se existe um isomorfismo $T$ de $E$ sobre $F$.

2. Um operador $T \in L(E, F)$ é dito uma isometria entre $E$ e $F$ se $\|T x\|=\|x\|$, para todo $x \in E$. Os espaços $E$ e $F$ são ditos isométricos se existe uma isometria $T$ de $E$ sobre $F$.

Definições 1.1.5. Sejam $E$ e $F$ espaços de Banach sobre $\mathbb{K}$.

1. Dizemos que $F$ possui uma cópia isomorfa de $E$ se existe um subespaço $M$ de $F$ que é isomorfo a E, ou seja, existe um isomorfismo de $E$ sobre $M$. Notação: denotamos por $E \hookrightarrow F$, se $F$ possui uma cópia isomorfa de $E$ e por $E \nrightarrow F$ se $F$ não tem uma cópia isomorfa de E.

2. Dizemos que $F$ possui uma cópia isométrica de $E$ se existe um subespaço $M$ de $F$ que é isométrico a $E$, ou seja, existe uma isometria de $E$ sobre $M$.

Definição 1.1.6. Sejam $E$ e $F$ espaços de Banach, e $T: E \rightarrow F$ um operador linear contínuo. Denotaremos por $T^{*}: F^{\prime} \rightarrow E^{\prime}$ o adjunto de $T$, definido por $T^{*}\left(y^{\prime}\right)=y^{\prime} \circ T$ para cada $y^{\prime}$ em $F^{\prime}$.

Proposição 1.1.7. Sejam E um espaço de Banach e $C: E \rightarrow E^{\prime \prime}$ a aplicação linear definida por $C(x)\left(x^{\prime}\right)=C_{x}\left(x^{\prime}\right)=x^{\prime}(x)$ para cada $x \in E$ e cada $x^{\prime} \in E^{\prime}$. Então a aplicação $C$ é uma isometria de $E$ em $E^{\prime \prime}$ e é chamada de inclusão natural.

Demonstração: Seja $x \in E$. Então $\|C(x)\|=\sup \left\{\left|C(x)\left(x^{\prime}\right)\right|: x^{\prime} \in B_{E^{\prime}}\right\}=$ $\sup \left\{\left|x^{\prime}(x)\right|: x^{\prime} \in B_{E^{\prime}}\right\}$ e pelo corolário 1.1.3, segue que $\|C(x)\|=\|x\|$. Assim a aplicação $C$ é uma isometria de $E$ em $E^{\prime \prime}$. 
Proposição 1.1.8. Sejam $E$ e $F$ espaços de Banach, e $T: E \rightarrow F$ um operador linear continuo. Sejam $C_{E}$ e $C_{F}$ as inclusões naturais de $E$ e $F$ em seus biduais respectivamente. Então $\left(T^{* *} \circ C_{E}\right)(E) \subseteq C_{F}(F)$ e $T^{* *} \circ C_{E}=T$.

Demonstração: Seja $x \in E$. Para cada $y^{\prime} \in F^{\prime},\left(\left(T^{* *} \circ C_{E}\right)(x)\left(y^{\prime}\right)\right)=$ $\left(C_{E}(x)\right)\left(T^{*}\left(y^{\prime}\right)\right)=\left(T^{*}\left(y^{\prime}\right)\right)(x)=y^{\prime}(T(x))$. Como $y^{\prime}(T(x))=C_{F}(T(x))\left(y^{\prime}\right)$, segue que $\left(T^{* *} \circ C_{E}\right)(x)=C_{F}(T(x))$ e daí o resultado.

O teorema da aplicação aberta que enunciamos a seguir tem várias aplicações. Apresentamos em seguida, uma das suas importantes aplicações que é o teorema do gráfico fechado, resultado esse que será utilizado no decorrer desta dissertação para mostrar a continuidade de operadores que construiremos.

Teorema 1.1.9 (Teorema da Aplicação Aberta). Sejam E e F espaços de Banach e $T: E \rightarrow F$ um operador linear contínuo sobrejetor. Então para todo aberto $G$ em $E$, $T(G)$ é um aberto em $F$.

Demonstração: Ver Megginson [16], pág. 43, teorema 1.6.5.

Teorema 1.1.10 (Teorema do Gráfico Fechado). Sejam E e F espaços de Banach, e $T: E \rightarrow F$ um operador linear. Se o gráfico de $T, G_{T}=\{(x, T x): x \in E\}$, é fechado, então $T$ é contínuo.

Demonstração: Consideremos $E \times F$ munido da seguinte norma

$$
\|(x, y)\|=\|x\|+\|y\| .
$$

Como $E \times F$ é um espaço de Banach e $G_{T}$ é fechado, segue que $G_{T}$ é um espaço de Banach.

Sejam $P_{1}: G_{T} \rightarrow E$ e $P_{2}: G_{T} \rightarrow F$ operadores lineares dados por $P_{1}(x, T x)=x$ e $P_{2}(x, T x)=T x . \mathrm{De}$

$$
\begin{gathered}
\left\|P_{1}(x, T x)\right\|=\|x\| \leq\|x\|+\|T x\|=\|(x, T x)\| \\
\left\|P_{2}(x, T x)\right\|=\|T x\| \leq\|x\|+\|T x\|=\|(x, T x)\|
\end{gathered}
$$

segue que $P_{1}$ e $P_{2}$ são contínuos. Além disso, temos que $P_{1}$ é bijetor, e logo, pelo Teorema da Aplicação Aberta (teorema 1.1.9), $P_{1}^{-1}$ é contínuo. Como $T=P_{2} \circ P_{1}^{-1}$ segue que $T$ é também contínuo. 
Definição 1.1.11. Seja $X$ um espaço métrico. Um subconjunto $M$ de $X$ é dito

a) raro em $X$ se $\bar{M}$ tem interior vazio.

b) de primeira categoria em $X$ se $M$ é união enumerável de conjuntos raros em $X$.

c) de segunda categoria em $X$ se $M$ não é de primeira categoria em $X$.

Teorema 1.1.12. Seja $X$ um espaço métrico completo não vazio. Então todo subconjunto de $X$ aberto, não vazio, é de segunda categoria em $X$. Em particular, o espaço $X$ é de segunda categoria em $X$.

Teorema 1.1.13 (Princípio da Limitação Uniforme). Sejam E um espaço de Banach, $F$ um espaço normado e $\mathcal{F}$ uma família não vazia de operadores lineares contínuos de $E$ em F. Se $\sup \{\|T x\|: T \in \mathcal{F}\}<\infty$ para cada $x \in E$, então $\sup \{\|T\|: T \in \mathcal{F}\}<\infty$.

Demonstração: Para cada $k \in \mathbb{N}$, seja $A_{k} \subset E$ o conjunto dos $x \in E$ tais que $\left\|T_{n} x\right\| \leq k \forall n \in \mathbb{N}$. Observamos que cada $A_{k}$ é fechado. De fato, para cada $x \in \overline{A_{k}}$, existe uma seqüência $\left(x_{j}\right)_{j} \subset A_{k} \operatorname{com} x_{j} \rightarrow x$. Assim para cada $n$ fixo, temos que $\left\|T_{n} x_{j}\right\| \leq k$. Agora como $T_{n}$ é contínuo, obtemos $\left\|T_{n} x\right\| \leq k$. Logo $x \in A_{k}$ e $A_{k}$ é fechado.

Para cada $x \in E$ temos que $\sup \{\|T x\|: T \in \mathcal{F}\}<\infty$, assim cada $x \in E$ pertence a algum $A_{k}$. Logo $E=\cup_{k=1}^{\infty} A_{k}$. Como $E$ é completo, pelo teorema 1.1.12 temos que algum $A_{k}$ contém uma bola aberta, ou seja, existe $x_{0} \in E$ e $r>0$ tais que $B\left(x_{0}, r\right) \subset A_{k_{0}}$.

Se $x \in E$ é qualquer, $x \neq 0$, seja $z=x_{0}+\frac{r x}{2\|x\|}$. Então $\left\|z-x_{0}\right\|<r$ e logo $z \in B\left(x_{0}, r\right)$. Como $B\left(x_{0}, r\right) \subset A_{k_{0}}$, segue que $\left\|T_{n} z\right\| \leq k_{0} \forall n$ e também que $\left\|T_{n} x_{0}\right\| \leq k_{0}$.

Para cada $n,\left\|T_{n} x\right\|=\frac{2\|x\|}{r}\left\|T_{n}\left(z-x_{0}\right)\right\| \leq \frac{4 k_{0}\|x\|}{r}$. Assim segue que $\left\|T_{n}\right\|=\sup \left\{\left\|T_{n} x\right\|:\|x\|=1\right\} \leq \frac{4 k_{0}}{r}$, e segue o resultado.

Definição 1.1.14. Seja $E$ um espaço de Banach. Um subespaço $M$ de $E$ é dito complementado em $E$ se $M$ é fechado em $E$ e existe um subespaço fechado $N$ de $E$ tal que $E=M \oplus N$.

Se $H$ é um espaço de Hilbert e $F$ é um subespaço fechado de $H$, então $F$ é complementado em $H$ e $H=F \oplus F^{\perp}$ com $F^{\perp}$ o complemento ortogonal de $F$.

Definição 1.1.15. Seja $X$ um espaço vetorial. Um operador linear $P: X \rightarrow X$ é chamado uma projeção em $X$ se $P(P x)=P(x)$ para cada $x \in X$, isto é, $P^{2}=P$. 
Como primeira aplicação do teorema do gráfico fechado, a seguinte proposição mostra que existe uma correspondência bijetora entre projeções $P$ de $E$ sobre $M$ e os subespaços fechados de $E, N$, tais que $E=M \oplus N$.

Proposição 1.1.16. Um subespaço $M$ de um espaço de Banach E é complementado em E se, e somente se, é a imagem de uma projeção contínua em E.

Demonstração: Suponhamos que $M$ é complementado em $E$. Então existe $N$ subespaço fechado de $E$ tal que $E=M \oplus N$. Logo cada $x \in E$ tem uma representação única da forma $x=m+n, m \in M$ e $n \in N$.

Seja $P$ um operador de $E$ em $E$ com $\operatorname{Im} P=M$ dado por $P(m+n)=m$. Temos que $P$ é linear e $P^{2}=P$. Vamos mostrar que $P$ é contínuo. Suponhamos que

$$
x_{k}=m_{k}+n_{k} \rightarrow x, m_{k} \in M, n_{k} \in N \text { e P } x_{k}=m_{k} \rightarrow y
$$

Como $M$ é fechado, $y \in M$. Logo $y=P y$ e $n_{k} \rightarrow x-y$. Como $N$ também é fechado, $x-y \in N$. Portanto $0=P(x-y)=P x-y$. Segue do Teorema do Gráfico Fechado (teorema 1.1.10), que $P$ é contínuo.

Reciprocamente, suponhamos que $P$ é uma projeção contínua em $E \operatorname{com} \operatorname{Im} P=M$. Seja $N=\operatorname{ker}(P)$. Como $P$ é contínuo, $N$ é fechado. Como $P$ é uma projeção contínua de $E$ sobre $M, M$ também é fechado. Além disso $N \cap M=\{0\}$, pois $v \in N \cap M$ implica que $v=P v=0$. Como todo $x \in E$ pode ser escrito como $x=P x+(x-P x)$ e $x-P x \in N$, segue que $E=M+N$. Logo $M$ é complementado em $E$.

Lembramos que se $M$ é um subespaço de um espaço vetorial $X$, então a codimensão de $M$ em $X$ é a dimensão do espaço vetorial quociente $X / M$. Denotaremos por $\dot{x}$ a classe de equivalência de $x$ em $X / M$. Se $X$ é um espaço normado e $M$ é um subespaço fechado de $X$, então definimos uma norma em $X / M$ dada por $\|\dot{x}\|=\inf \{\|y\|: y \in \dot{x}\}$.

O teorema que enunciamos a seguir garante que todo subespaço fechado de $E$ de codimensão finita de um espaço de Banach $E$ é complementado em $E$. Utilizaremos esse teorema ainda neste capítulo na demonstração de resultados que caracterizam operadores compactos.

Teorema 1.1.17. Sejam $E$ um espaço de Banach e $M$ um subespaço fechado de $E$ de codimensão finita. Então $M$ é complementado em $E$. 
Demonstração: Seja $\left\{\dot{x}_{1}, \ldots, \dot{x}_{n}\right\}$ uma base de $E / M$ e seja $N=\left[x_{1}, \ldots, x_{n}\right]$. Assim $N$ é um subespaço fechado de $E$. Sejam $f_{1}, \ldots, f_{n} \in E^{\prime}$ funcionais tais que $f_{i}\left(x_{j}\right)=\delta_{i j}$, isto é, a base dual de $\left[x_{1}, \ldots, x_{n}\right]$ estendida por Hahn-Banach a $E$.

Considere $\Pi: E \rightarrow E / M$ dada por $\Pi(v)=\sum_{i=1}^{n} f_{i}(v) \dot{x}_{i}$. $\Pi$ é uma projeção contínua. Para cada $v \in E$ considere $w=v-\sum_{i=1}^{n} f_{i}(v) x_{i}$. Afirmamos que $w \in M$. De fato $\Pi(w)=\Pi(v)-\sum_{i=1}^{n} f_{i}(v) \Pi\left(x_{i}\right)=\sum_{i=1}^{n} f_{i}(v) \dot{x}_{i}-\sum_{i=1}^{n} f_{i}(v) \dot{x}_{i}=0$, isto é, $w \in M$. Portanto $v=w+\sum_{i=1}^{n} f_{i}(v) x_{i} \in M+N$. Se $u \in M \cap N$, então $u=\sum_{i=1}^{n} \alpha_{i} x_{i}$ e $\Pi(u)=\sum_{i=1}^{n} \alpha_{i} \dot{x}_{i}=0$, pois $u \in M$. Portanto $\alpha_{i}=0$ para $i=1, \ldots n$. Donde $M$ é um subespaço complementado em $E$.

Definições 1.1.18. (Os espaços $\ell_{p}^{\prime} s$ e $c_{0}$ )

Dado $p$ qualquer, $1 \leq p<\infty$, considere $\ell_{p}$ como sendo o conjunto das seqüências $x=\left(x_{k}\right)_{k} \subset \mathbb{K}$ tais que a série $\sum_{k=1}^{\infty}\left|x_{k}\right|^{p}$ converge.

As operações usuais de adição e multiplicação por escalar em $\mathbb{K}^{\mathbf{N}}$ fazem de $\ell_{p}$ um espaço vetorial e a aplicação $\|\cdot\|_{p}$ de $\ell_{p}$ em $\mathbb{R}_{+}$dada por $\|x\|_{p}=\left(\sum_{k=1}^{\infty}\left|x_{k}\right|^{p}\right)^{1 / p}$ constitui uma norma em $\ell_{p}$ que o faz completo, ou seja, $\left(\ell_{p},\|\cdot\|_{p}\right)$ é um espaço de Banach.

Para $p=\infty$, considere $\ell_{\infty}$ como sendo o conjunto das seqüências $x=\left(x_{k}\right)_{k} \subset \mathbb{K}$ limitadas.

As operações usuais de adição e multiplicação por escalar em $\mathbb{K}^{\mathbf{N}}$ fazem de $\ell_{\infty}$ um espaço vetorial, e a aplicação no espaço a valores em $\mathbb{R}$ dada por $\|x\|_{\infty}=\sup _{k \in N}\left|x_{k}\right|$ constitui uma norma em $\ell_{\infty}$ que o faz completo, ou seja, $\left(\ell_{\infty},\|\cdot\|_{\infty}\right)$ é um espaço de Banach.

Definimos $c_{0}$ como sendo o subespaço de $\ell_{\infty}$ constituído das seqüências $x=\left(x_{k}\right)_{k} \subset \mathbb{K}$ que convergem a zero.

Munindo co da norma induzida de $\ell_{\infty}$, o espaço normado obtido é Banach.

Para $1 \leq p<q<\infty$, temos que $\ell_{p} \subset \ell_{q}$

Se E é co ou $\ell_{p}$, com $1 \leq p<\infty$, considere a seqüência $\left(e_{n}\right)_{n}$ com $e_{n}=$ $(0, \ldots, 0,1,0, \ldots)$. Esta seqüência será chamada de seqüência de vetores unitários de E.

Definição 1.1.19. (O espaço $C(\Omega)$ ) 
Dado $\Omega$ um espaço de Hausdorff compacto, considere $C(\Omega)$ o conjunto das funçôes continuas $f$ de $\Omega$ em $\mathbb{K}$.

As operações usuais de adição e multiplicação por escalar dos espaços de funções fazem de $C(\Omega)$ um espaço vetorial, e a aplicação $\|\cdot\|_{\infty}$ de $C(\Omega)$ em $\mathbb{R}_{+}$dada por $\|f\|_{\infty}=\sup \{|f(x)|: x \in \Omega\}$ constitui uma norma em $C(\Omega)$ que o faz completo, ou seja, $\left(C(\Omega),\|\cdot\|_{\infty}\right)$ é um espaço de Banach.

No capítulo 3, estudamos algumas caracterizações dos espaços de Banach $F$ para os quais todos os operadores contínuos de $C(\Omega)$ em $F$ sejam compactos, com $\Omega$ um espaço de Hausdorff compacto. Estas caracterizações dependem de $\Omega$ ser disperso ou não. A seguir apresentamos a definição de espaço topológico disperso e alguns resultados que utilizaremos.

Definição 1.1.20. (Espaços dispersos)

Um espaço topológico $S$ é dito disperso se todo subconjunto fechado, não vazio de $S$, munido da topologia induzida, tem um ponto isolado.

Como exemplo, considere $S=\{0,1,1 / 2,1 / 3, \ldots\}$ com a topologia induzida de $\mathbb{R}$. Então $S$ é disperso.

Teorema 1.1.21. Seja $\Omega$ um conjunto infinito. Se $\Omega$ é um espaço de Hausdorff compacto disperso, então $C(\Omega)$ contém um subespaço complementado que é isométrico a $c_{0}$.

Demonstração: Ver Rosenthal [23], pág. 201, demonstração do corolário 3.2.

Definição 1.1.22. Seja $S$ um conjunto. Considere $\ell_{1}(S)$ como sendo a família de todas as funções $f: S \rightarrow \mathbb{K}$ tais que $\sum_{s \in S}|f(s)|<\infty$, com a soma definida por $\sum_{s \in S}|f(s)|=\sup \left\{\sum_{s \in F}|f(s)|: F\right.$ é um subconjunto finito de $\left.S\right\}$. As operaçôes usuais de adição e multiplicação fazem de $\ell_{1}(S)$ um espaço vetorial e a aplicação $\|\cdot\|$ de $\ell_{1}(S)$ em $\mathbb{R}_{+}$dada por $\|f\|=\sum_{s \in S}|f(s)|$ constitui uma norma em $\ell_{1}(S)$ que o faz completo, ou seja, $\left(\ell_{1}(S),\|\cdot\|\right)$ é um espaço de Banach.

Teorema 1.1.23. Sejam $S$ um conjunto. Se $S$ é um espaço de Hausdorff compacto disperso, então $C(S)^{\prime}$ é isomorfo a $\ell_{1}(S)$.

Demonstração: Ver Semadeni [24], pág. 338, corolário 19.7.7. 
Definição 1.1.24. Seja $X$ um espaço normado. Dizemos que $X$ é separável se admite um subconjunto enumerável denso.

Teorema 1.1.25. Seja $\Omega$ um conjunto infinito. Se $\Omega$ é um espaço de Hausdorff compacto não disperso, então todo espaço de Banach separável é isométrico a um subespaço de $C(\Omega)$.

Demonstração: Ver Rosenthal [23], pág. 201, demonstração do corolário 3.2.

Teorema 1.1.26. Seja E um espaço de Banach separável que contém uma cópia isomorfa de $c_{0}$. Então existe uma projeção de norma $\leq 2$ de $E$ sobre $c_{0}$.

Demonstração: Ver Lindenstrauss, Tzafiri [14], pág. 106, teorema 2.f.5.

No capítulo 3, mostramos que se $F$ é um espaço de Banach para o qual cada operador linear contínuo de $C(\Omega)$ em $F$ é absolutamente 2-somante, com $\Omega$ um espaço de Hausdorff compacto não disperso, então cada operador linear contínuo de $C(\Omega)$ em $F$ é compacto se, e somente se, cada operador linear contínuo de $\ell_{2}$ em $F$ é compacto. Apresentamos a seguir a definição de operadores absolutamente 2-somantes e alguns resultados.

Definição 1.1.27. (Operadores absolutamente 2-somantes)

Sejam E e $F$ espaços de Banach. Um operador $T \in L(E, F)$ é dito absolutamente 2-somante se dada uma seqüência $\left(x_{n}\right)_{n}$ qualquer em $E$ tal que $\sum\left|f\left(x_{n}\right)\right|^{2}<\infty$ para cada $f \in E^{\prime}$, temos que $\sum\left\|T x_{n}\right\|^{2}<\infty$.

Denotamos por $\Pi_{2}(E, E)$ o conjunto dos operadores absolutamente 2-somantes.

Em seguida apresentamos o teorema de fatoração de Grothendieck-Pietsch que estabelece que todo operador absolutamente 2-somante se fatora através de um espaço de Hilbert.

Teorema 1.1.28 (Grothendieck-Pietsch). Sejam E e F espaços de Banach. Então cada operador $T \in \Pi_{2}(E, F)$ admite uma fatoração através de um espaço de Hilbert.

Demonstração: Ver Retherford [22], pág. 105, teorema de Grothendieck-Pietsch. 
Teorema 1.1.29. Sejam $X$ um subespaço fechado de $c_{0}, \Omega$ um espaço de Hausdorff compacto e $T$ um operador linear contínuo de $X$ em $C(\Omega)$. Então para todo $\epsilon>0, T$ estende-se para um operador contínuo $\hat{T}$ de $c_{0}$ em $C(\Omega)$ com $\|\hat{T}\|<(1+\epsilon)\|T\|$.

Demonstração: Ver Lindenstrauss, Pelczynski [13], pág. 231, teorema 3.1.

$\mathrm{Na}$ quinta seção deste capítulo apresentamos alguns resultados para o espaço de todos os operadores compactos entre o espaço $\ell_{2}$. Para tal vamos precisar da seguinte proposição que estabelece que o espaço $L\left(\ell_{2}, \ell_{2}\right)$ contém uma cópia isométrica de $\ell_{\infty}$.

Proposição 1.1.30. O espaço $L\left(\ell_{2}, \ell_{2}\right)$ contém uma cópia isométrica de $\ell_{\infty}$.

Demonstração: Seja $\varphi: \ell_{\infty} \rightarrow L\left(\ell_{2}, \ell_{2}\right)$ a aplicação linear dada por $\varphi(a)(x)=$ $T(a)(x)=\left(a_{i} x_{i}\right)_{i}$ para cada $a=\left(a_{i}\right)_{i} \in \ell_{\infty}$ e cada $x=\left(x_{i}\right)_{i} \in \ell_{2}$.

Como

$$
\left(\sum\left|a_{i} x_{i}\right|^{2}\right)^{1 / 2}=\left(\sum\left|a_{i}\right|^{2}\left|x_{i}\right|^{2}\right)^{1 / 2} \leq\|a\|_{\infty}\left(\sum\left|x_{i}\right|^{2}\right)^{1 / 2}=\|a\|_{\infty}\|x\|_{2},
$$

segue que $T(a)$ está bem definido e $\|T(a)\| \leq\|a\|_{\infty}$, portanto $T(a)$ é contínuo.

Agora, dado $\epsilon>0$, existe $n_{0} \in \mathbb{I}$ tal que $\left|a_{n_{0}}\right|>\|a\|_{\infty}-\epsilon$. Como

$$
\left\|T(a)\left(e_{n_{0}}\right)\right\|_{2}=\left|a_{n_{0}}\right|>\|a\|_{\infty}-\epsilon
$$

segue que $\|T(a)\|>\|a\|_{\infty}-\epsilon$, fazendo $\epsilon \rightarrow 0$, temos que $\|T(a)\| \geq\|a\|_{\infty}$. Logo $\|T(a)\|=\|a\|_{\infty}$ e conseqüentemente $\varphi$ é uma isometria.

Teorema 1.1.31 (Sobczysk). Seja $X$ um subespaço fechado de um espaço de Banach separável $E$. Se $X$ é isomorfo a c $c_{0}$, então $X$ é complementado em $E$.

Demonstração: Ver [9], pág. 142, teorema 5.14 .

$\mathrm{Na}$ quinta seção deste capítulo apresentamos alguns resultados para o espaço $K\left(\ell_{2}, \ell_{2}\right)$ de todos os operadores compactos entre o espaço $\ell_{2}$. Mostramos que $K\left(\ell_{2}, \ell_{2}\right)$ não é complementado em $L\left(\ell_{2}, \ell_{2}\right)$, para isso usaremos o seguinte teorema que mostra que o espaço $c_{0}$ não é complementado em $\ell_{\infty}$.

Teorema 1.1.32 (Phillips). O espaço c coño é complementado em $\ell_{\infty}$. 
Demonstração: Ver Megginson [16], pág. 301, teorema 3.2.20.

Sejam $E$ um espaço de Banach e $A$ um subconjunto não vazio de $E$. A envoltória convexa fechada de $A$ é o conjunto $\overline{c o}(A)=\left\{\sum_{i=1}^{\infty} \lambda_{i} x_{i}: x_{i} \in A, \lambda_{i} \geq 0\right.$ e $\left.\sum_{i=1}^{\infty} \lambda_{i}=1\right\}$.

O próximo teorema que utilizaremos na demonstração de resultados no capítulo 2 mostra que todo subconjunto compacto de um espaço normado está contido na envoltória convexa fechada de uma seqüência que converge em norma para zero.

Teorema 1.1.33. Sejam $X$ um espaço normado e $K$ um subconjunto de $X$. Se $K$ é compacto, então existe uma seqüência $\left(x_{n}\right)_{n} \subset X$ tal que $\lim _{n}\left\|x_{n}\right\|=0$ e Kᄃ $\overline{c o}\left(\left(x_{n}\right)_{n}\right)$.

Demonstração: Ver Diestel [5], pág. 3, teorema 5.

Terminamos essa seção apresentando o Lema de Riesz que utilizaremos no capítulo 3.

Lema 1.1.34 (Lema de Riesz). Sejam $Y$ um subespaço próprio fechado de um espaço normado $X$ e $0<\theta<1$. Então existe um $x_{\theta} \in S_{X}$ tal que $\left\|x_{\theta}-y\right\|>\theta$ para todo $y \in Y$.

Demonstração: Tomemos $x \in X \backslash Y$. Como $Y$ é fechado, a distância de $x$ a $Y$, $d$, é positiva, isto é,

$$
0<d=\inf \{\|x-y\|: y \in Y\}
$$

Para $0<\theta<1, d / \theta>d$ e assim, existe um $z \in Y$ tal que $\|x-z\|<d / \theta$.

Seja $x_{\theta}=\frac{x-z}{\|x-z\|}$. Temos que $x_{\theta} \in S_{X}$, e além disso, se $y \in Y$, então

$$
\begin{gathered}
\left\|x_{\theta}-y\right\|=\left\|\frac{x-z}{\|x-z\|}-y\right\|= \\
=\left\|\frac{x}{\|x-z\|}-\frac{z}{\|x-z\|}-\frac{\|x-z\| y}{\|x-z\|}\right\|= \\
=\frac{1}{\|x-z\|}\|x-\underbrace{(z+\|x-z\| y)}_{\text {um elemento de } Y}\|> \\
\frac{\theta}{d} d=\theta .
\end{gathered}
$$




\subsection{Topologia fraca em espaços de Banach}

Nesta dissertação, utilizaremos as definições e algumas propriedades de topologia fraca e operadores fracamente compactos. A topologia fraca em um espaço normado $X$ é a menos fina que torna os elementos do dual de $X$ contínuos. Essa topologia não é induzida por uma métrica, logo argumentos familiares usados para espaços métricos baseados em convergência de seqüências não podem ser utilizados na sua forma usual. Entretanto a maioria dos resultados pode ser adaptada trocando-se seqüências por redes. Começamos então esta seção com uma discussão sobre redes.

Definição 1.2.1. Um conjunto dirigido é um conjunto não-vazio I com uma relação tal que:

(1) $\alpha \preceq \alpha$ para todo $\alpha \in I$.

(2) $\alpha \preceq \beta$ e $\beta \preceq \gamma \Rightarrow \alpha \preceq \gamma$ para todos $\alpha, \beta$, e $\gamma \in I$.

(3) Para todos $\alpha$ e $\beta \in I$ existe um $\gamma_{\alpha, \beta} \in I$ tal que $\alpha \preceq \gamma_{\alpha, \beta}$ e $\beta \preceq \gamma_{\alpha, \beta}$.

Definição 1.2.2. Uma rede em um conjunto $X$ é uma função $\alpha \in I \mapsto x_{\alpha} \in X$, com $I$ um conjunto dirigido. Analogamente às seqüências, denotamos uma rede $\alpha \in I \mapsto x_{\alpha} \in X$ $\operatorname{por}\left(x_{\alpha}\right)_{\alpha \in I}$ ou $\left(x_{\alpha}\right)$.

Exemplos 1.2.3. a) Toda seqüência é uma rede, com o conjunto de índices IN com sua ordem natural.

b) $O$ conjunto $\mathbb{R}$ com sua ordem natural é um conjunto dirigido, logo toda funçấo com domínio em $\mathbb{R}$ é uma rede.

Definição 1.2.4. Seja $\left(x_{\alpha}\right)_{\alpha \in I}$ uma rede em um espaço topológico $X$ e $x \in X$. Dizemos que $\left(x_{\alpha}\right)_{\alpha \in I}$ converge para $x$ se para cada vizinhança aberta $V$ de $x$ em $X$, existe $\alpha_{0}$ em I tal que $\alpha_{0} \preceq \alpha \Rightarrow x_{\alpha} \in V$.

Se $X$ é um espaço de Hausdorff, então dados dois pontos $x, y \in X$ distintos, existem vizinhanças abertas $V_{x}$ e $V_{y}$ de $x$ e y respectivamente tais que $V_{x} \cap V_{y}=\emptyset$. Se uma rede $\left(x_{\alpha}\right)_{\alpha \in I} \subset X$ converge para $x$ e $y$, existem $\alpha_{1}$ e $\alpha_{2}$ em I tais que $\alpha_{1} \preceq \alpha \Rightarrow x_{\alpha} \in V_{x}$ $e \alpha_{2} \preceq \alpha \Rightarrow x_{\alpha} \in V_{y}$. Sendo I um conjunto dirigido, é possivel encontrar $\alpha_{0} \in I$ tal que $\alpha_{1} \preceq \alpha_{0}$ e $\alpha_{2} \preceq \alpha_{0}$. Devemos ter $x_{\alpha_{0}} \in V_{x} \cap V_{y}$. Logo, uma rede em um espaço de Hausdorff tem apenas um limite. Como iremos trabalhar apenas com espaços de Hausdorff, escrevemos $x_{\alpha} \rightarrow x$ para denotar que a rede $\left(x_{\alpha}\right)_{\alpha \in I}$ converge para $x$. 
Proposição 1.2.5. Sejam $X$ e $Y$ espaços topológicos, e $f: X \rightarrow Y$ uma função. Então $f$ é contínua em $x \in X$ se, e somente se, $x_{\alpha} \rightarrow x \Rightarrow f\left(x_{\alpha}\right) \rightarrow f(x)$, para toda rede $\left(x_{\alpha}\right)_{\alpha \in I} \subset X$.

Demonstração: Sejam $f: X \rightarrow Y$ contínua em $x$ e $x_{\alpha} \rightarrow x$. Para toda vizinhança $V$ de $f(x)$, existe uma vizinhança $U$ de $x$ tal que $f(U) \subset V$. Como $\left(x_{\alpha}\right)_{\alpha \in I}$ converge para $x$, existe $\alpha_{0}$ em $I$ tal que $\alpha_{0} \preceq \alpha \Rightarrow x_{\alpha} \in U$. Logo $\alpha_{0} \preceq \alpha \Rightarrow x_{\alpha} \in U \Rightarrow f\left(x_{\alpha}\right) \in V$.

Por outro lado, suponhamos que $x_{\alpha} \rightarrow x \Rightarrow f\left(x_{\alpha}\right) \rightarrow f(x)$, para toda rede $\left(x_{\alpha}\right)_{\alpha \in I} \subset X$ mas $f: X \rightarrow Y$ não é contínua em $x$. Então, existe uma vizinhança $V$ de $f(x)$ tal que para toda vizinhança $U$ de $x, f(U) \not \subset V$. Pelo axioma da escolha, para cada vizinhança $U$ de $x$ podemos tomar $x_{U} \in U$ tal que $f\left(x_{U}\right) \notin V$. O conjunto $\Upsilon_{x}$ de todas as vizinhanças de $x$ em $X$ é um conjunto dirigido pela relação $W \leq U \Leftrightarrow U \subset W$. Logo, $\left(x_{U}\right)_{U \in \Upsilon_{x}}$ é uma rede em $X$ que converge para $x$, pois dado $U \in \Upsilon_{x}$, tomando $U_{0}=U$ temos que $W \geq U \Rightarrow W \subset U \Rightarrow x_{W} \in U$. Evidentemente, a rede $\left(f\left(x_{U}\right)\right)_{U \in \Upsilon}$ não converge para $f(x)$, o que é uma contradição.

Apresentamos a seguir a definição de topologia fraca e alguns resultados que utilizaremos posteriormente.

Definição 1.2.6. (Topologia fraca)

Seja X um espaço normado. A topologia fraca de $X$ é a topologia obtida tomando como base todos os conjuntos da forma:

$$
V\left(x_{0}, x_{1}^{\prime}, \ldots, x_{n}^{\prime}, \epsilon\right)=\left\{x \in X: \sup _{i=1, \ldots, n}\left|x_{i}^{\prime}(x)-x_{i}^{\prime}\left(x_{0}\right)\right|<\epsilon\right\}
$$

onde $x_{0} \in X ; x_{1}^{\prime}, \ldots, x_{n}^{\prime} \in X^{\prime}, n \in \mathbb{N}$ e $\epsilon>0$.

Aqui, fracamente aberto, fracamente fechado, fracamente compacto, função fracamente contínua, etc estão se referindo a conjunto aberto, fechado, compacto, função contínua, etc com relação à topologia fraca.

Proposição 1.2.7. Uma rede $\left(x_{\alpha}\right)_{\alpha \in I} \subset X$ em um espaço de Banach $E$ é fracamente convergente para $x$ se, e somente se, $f\left(x_{\alpha}\right) \rightarrow f(x)$ em $\mathbb{K}$, para todo $f \in E^{\prime}$.

Demonstração: Se $x_{\alpha} \stackrel{\omega}{\rightarrow} x$, então, dado $f \in E^{\prime}$, pela proposição 1.2 .5 segue que $f\left(x_{\alpha}\right) \rightarrow f(x)$. 
Por outro lado, se para todo funcional linear contínuo $f, f\left(x_{\alpha}\right) \rightarrow f(x)$, dado um aberto $V\left(x, f_{1}, f_{2}, \ldots, f_{n}, \epsilon\right)$, para cada $i=1,2, \ldots, n$, existe $\alpha_{i} \in I$ tal que $\left|f_{i}\left(x_{\alpha}\right)-f_{i}(x)\right|<\epsilon$, para todo $\alpha \geq \alpha_{i}$. Tomando $\alpha_{0} \geq \alpha_{i}$ para todo $i$, temos que

$$
\alpha \geq \alpha_{0} \Rightarrow\left|f_{i}\left(x_{\alpha}\right)-f_{i}(x)\right|<\epsilon(i=1,2, \ldots, n) \Rightarrow x_{\alpha} \in V\left(x, f_{1}, f_{2}, \ldots, f_{n}, \epsilon\right),
$$

e portanto $x_{\alpha} \stackrel{\omega}{\rightarrow} x$.

Exemplo 1.2.8. Seja $\left(e_{n}\right)_{n}$ a seqüência de vetores unitários de $\ell_{2}$. É fácil ver que $e_{n} \stackrel{\omega}{\rightarrow} 0$ e como $\left\|e_{n}\right\|=1$, para todo $n \in \mathbb{N}$, temos que $e_{n} \nrightarrow 0$ em $\ell_{2}$.

Proposição 1.2.9. Sejam $X$ um espaço normado e $\left(x_{n}\right)_{n}$ uma seqüência em $X$. Se $\left(x_{n}\right)_{n}$ é fracamente convergente, então $\left(x_{n}\right)_{n}$ é limitada.

Demonstração: Se $x_{n} \stackrel{\omega}{\rightarrow} x$, então $f\left(x_{n}\right) \rightarrow f(x)$ para cada $f \in X^{\prime}, \operatorname{logo}\left(f\left(x_{n}\right)\right)_{n} \subset$ $\mathbb{I K}$ é limitado. Assim para cada $f \in X^{\prime}$ existe $c_{f}$ tal que $\left|f\left(x_{n}\right)\right| \leq c_{f}$ para todo $n \in \mathbb{N}$.

Seja $C: X \rightarrow X^{\prime \prime}$ a inclusão natural. Então $\left|C_{x_{n}}(f)\right|=\left|f\left(x_{n}\right)\right| \leq c_{f}$, isto é, a sequiência $\left(\left|C_{x_{n}}(f)\right|\right)_{n}$ é limitada para todo $f \in X^{\prime}$. Pelo Princípio da Limitação Uniforme, teorema 1.1.13, $\left(\left\|C_{x_{n}}\right\|\right)_{n}$ é limitada, agora como $\left\|C_{x_{n}}\right\|=\left\|x_{n}\right\|$, segue que $\left(x_{n}\right)_{n}$ é limitada.

Teorema 1.2.10. Sejam E e $F$ espaços de Banach, e $T: E \rightarrow F$ um operador linear. Então $T$ é norma-norma contínuo se, e somente se, é fraco-fraco contínuo.

Demonstração: Suponhamos que $T$ é norma-norma contínuo. Sejam $x_{\alpha} \stackrel{\omega}{\rightarrow} x$ em $E$ e $y^{\prime} \in F^{\prime}$. Como $T$ é norma-norma contínuo, $y^{\prime} \circ T \in E^{\prime}$ e logo $y^{\prime} \circ T\left(x_{\alpha}\right) \rightarrow y^{\prime} \circ T(x)$, conseqüentemente $T x_{\alpha} \stackrel{\omega}{\rightarrow} T x$ em $F$. Logo $T$ é fraco-fraco contínuo.

Reciprocamente, seja $T: E \rightarrow F$ um operador fraco-fraco contínuo. Suponhamos por absurdo que $T$ não é norma-norma contínuo. Então existe uma seqüência $\left(x_{n}\right)_{n} \subset E$ tal que $x_{n} \rightarrow 0$ e $\left\|T x_{n}\right\| \geq n^{2}$ para todo $n \in \mathbb{N}$. Como $x_{n} \rightarrow 0$, segue que $x_{n} \stackrel{\omega}{\rightarrow} 0$ em $E, \mathrm{e}$ assim, por hipótese, $T x_{n} \stackrel{\omega}{\rightarrow} 0 \mathrm{em} F$. Agora pela proposição 1.2 .9 , a seqüência $\left(T x_{n}\right)_{n}$ é limitada, uma contradição. Portanto $T$ é norma-norma contínuo.

Definição 1.2.11. Seja $X$ um espaço normado. Dizemos que $X$ tem a propriedade de Schur se para toda seqüência $\left(x_{n}\right)_{n} \subset X$ tal que $x_{n} \stackrel{\omega}{\rightarrow} x$, para algum $x \in X$, implicar que $x_{n} \rightarrow x$ em $X$. 
Exemplo 1.2.12. No exemplo 1.2.8 acima foi mostrado que $\ell_{2}$ não tem a propriedade de Schur.

Proposição 1.2.13. Sejam $S$ um conjunto e $\ell_{1}(S)$ o espaço definido como em 1.1.22. Então $\ell_{1}(S)$ tem a propriedade de Schur.

Demonstração: Ver por exemplo Semadeni [24], pág. 303, teorema 17.7.5.

Teorema 1.2.14 (Teorema $\ell_{1}$ de Rosenthal). Seja E um espaço de Banach. Cada seqüência limitada $\left(x_{n}\right)_{n} \subset E$ admite uma subseqüência fracamente de Cauchy em E se, e somente se, $\ell_{1} \leftrightarrow E$.

Demonstração: Ver por exemplo Diestel [5], pág. 201.

Teorema 1.2.15. Seja $X$ um espaço de Banach. Então $X$ é reflexivo se, e somente se, toda seqüência limitada em $X$ admite uma subseqüência fracamente convergente.

Demonstração: Ver Megginson [16], pág. 119, teorema 1.13.5.

Teorema 1.2.16 (Eberlein-Smulian). Seja A um subconjunto de um espaço normado. Então $A$ é relativamente fracamente compacto se, e somente se, $A$ é relativamente fracamente seqüencialmente compacto.

Demonstração: Ver Megginson [16], pág. 248, teorema 2.8.6.

\subsection{Séries e Bases de Schauder}

Nesta seção apresentaremos resultados relacionados a séries e bases em espaços de Banach. Este tema será de suma importância no capítulo 2 para estudar algumas caracterizações de operadores compactos em espaços de Banach.

Sejam $X$ um espaço normado e $\left(x_{n}\right)_{n}$ uma seqüência em $X$. Então a série gerada por $\left(x_{n}\right)_{n}$ é a seqüência $\left(\sum_{n=1}^{m} x_{n}\right)_{m=1}^{\infty}$. Para cada inteiro positivo $m$, o $m$-ésimo termo $\sum_{n=1}^{m} x_{n}$ dessa seqüência de somas é a $m$ - ésima soma parcial da série. Se a série converge, isto é, se $\lim _{m} \sum_{n=1}^{m} x_{n}$ existe, então esse limite é a soma da série e é denotado por $\sum_{n=1}^{\infty} x_{n}$ ou por $\sum_{n} x_{n}$. 
Definições 1.3.1. Sejam $E$ um espaço normado e $\sum_{i=1}^{\infty} x_{i}$ uma série em E. Então a série $\sum_{i=1}^{\infty} x_{i}$ é:

(1) Incondicionalmente convergente se para cada permutação $\sigma: \mathbb{N} \rightarrow \mathbb{N}$ a série $\sum_{i=1}^{\infty} x_{\sigma(i)}$ converge.

(2) Somável para um $x \in E$ se para cada $\epsilon>0$ é possivel obter $F_{\epsilon}$ (finito) $\subset \mathbb{N}$ tal que para todo $F$ finito com $F_{\epsilon} \subset F \subset \mathbb{N}$, temos que $\left\|x-\sum_{i \in F} x_{i}\right\|<\epsilon$.

(3) Limitada multiplicada convergente se para cada seqüência de escalares $\left(\lambda_{i}\right)_{i}$ a série $\sum_{i=1}^{\infty} \lambda_{i} x_{i}$ converge.

(4) Cauchy somável se para cada $\epsilon>0$ é possivel obter $n_{0} \in \mathbb{N}$ tal que para todo subconjunto finito de $\mathbb{N}, F$, com $\min F \geq n_{0}$, temos que $\left\|\sum_{i \epsilon F} x_{i}\right\|<\epsilon$.

Proposição 1.3.2. Sejam E um espaço de Banach, $\sum_{i=1}^{\infty} x_{i}$ uma série em E e (1), (2), (3), (4) como na definição anterior. Então são equivalentes: (1) $\Leftrightarrow$ (2) $\Leftrightarrow$ (3) $\Leftrightarrow$ (4).

Demonstração: Para a implicação (1) $\Leftrightarrow(2)$, ver Retherford [22], pág. 14, para as implicações (1) $\Leftrightarrow(3) \Leftrightarrow(4)$, ver Diestel, Jarchow, Tonge [6], pág. 9, teorema 1.9.

Definição 1.3.3. Sejam $E$ um espaço de Banach e $\sum_{i=1}^{\infty} x_{i}$ uma série em E. Dizemos que a série $\sum_{i=1}^{\infty} x_{i}$ é absolutamente convergente se a série numérica $\sum_{i=1}^{\infty}\left\|x_{i}\right\|$ converge.

Em $\mathbb{K}$, convergência absoluta e convergência incondicional são equivalentes. Para espaços normados temos:

Proposição 1.3.4. Um espaço normado $X$ é um espaço de Banach se, e somente se, cada série absolutamente convergente em $X$ é também convergente.

Demonstração: Suponhamos que $X$ é um espaço de Banach e que $\sum_{i=1}^{\infty} x_{i}$ é uma série absolutamente convergente em $X$. Então para $s_{n}=\sum_{i=1}^{n} x_{i}$,

$$
\left\|s_{n+k}-s_{n}\right\|=\left\|\sum_{i=n+1}^{n+k} x_{i}\right\| \leq \sum_{i=n+1}^{n+k}\left\|x_{i}\right\| \rightarrow 0 \text { quando } n \rightarrow \infty
$$

Logo, $\left(s_{n}\right)_{n}$ é uma seqüência de Cauchy e portanto convergente no espaço de Banach $X$.

Suponhamos que $X$ não é um espaço de Banach. Seja $\left(x_{n}\right)_{n}$ uma seqüência de Cauchy não convergente em $X$. Para cada inteiro positivo $j$, existe um inteiro positivo $n_{j}$ tal que 
$\left\|x_{n}-x_{m}\right\| \leq 2^{-j}$ se $n, m \geq n_{j}$. Podemos assumir que $n_{j+1} \geq n_{j}$ para cada $j$. Como o limite de uma subseqüência de uma seqüência de Cauchy deve ser o limite da seqüência, a subseqüência $\left(x_{n_{j}}\right)_{j}$ não tem limite. Logo a série $\sum_{j=1}^{\infty}\left(x_{n_{j+1}}-x_{n_{j}}\right)$ não é convergente pois $\sum_{j=1}^{k}\left(x_{n_{j+1}}-x_{n_{j}}\right)=x_{n_{k+1}}-x_{n_{1}}$ para cada inteiro positivo $k$. Entretanto essa série é absolutamente convergente pois $\sum_{j=1}^{\infty}\left\|x_{n_{j+1}}-x_{n_{j}}\right\| \leq \sum_{j=1}^{\infty} 2^{-j}=1$.

Proposição 1.3.5. Sejam $E$ um espaço de Banach e $\sum_{n} x_{n}$ uma série em $E$. Se a série $\sum_{n} x_{n}$ é incondicionalmente convergente em $E$, então a série $\sum_{n} \alpha_{n} x_{n}$ também é incondicionalmente convergente em $E$ para todo $\left(\alpha_{n}\right)_{n} \in \ell_{\infty}$.

Demonstração: Se a série $\sum_{n} x_{n}$ é incondicionalmente convergente em $E$, pela proposição 1.3.2, a série $\sum_{n=1}^{\infty} x_{n}$ é limitada multiplicada convergente; conseqüentemente a série $\sum_{n} \alpha_{n} x_{n}$ é incondicionalmente convergente em $E$ para todo $\left(\alpha_{n}\right)_{n} \in \ell_{\infty}$.

Apresentamos também a definição de série $\omega^{*}$ incondicionalmente convergente.

Definição 1.3.6. (Séries $\omega^{*}$ incondicionalmente convergentes)

Seja $E$ um espaço de Banach. Uma série $\sum g_{n}$ no espaço dual $E^{\prime}$ de $E$ é dita $\omega^{*}$ incondicionalmente convergente se $\sum g_{n}(x)$ é incondicionalmente convergente para cada $x$ em $E$, ou equivalentemente, $\sum\left|g_{n}(x)\right|<\infty$ para cada $x$ em $E$.

Exemplos 1.3.7. (a) Para cada $n \in \mathbb{N}$, seja $p_{n}: \ell_{1} \rightarrow \mathbb{K}$ o funcional linear e contínuo definido por $p_{n}(\lambda)=\lambda_{n}$, para cada $\lambda=\left(\lambda_{j}\right)_{j} \in \ell_{1}$. Como $\sum\left|p_{n}(\lambda)\right|=\sum\left|\lambda_{n}\right|<\infty$ para cada $\lambda=\left(\lambda_{j}\right)_{j}$ em $\ell_{1}$, a série $\sum p_{n}$ é então $\omega^{*}$ incondicionalmente convergente em $\ell_{\infty}=\left(\ell_{1}\right)^{\prime}$.

(b) Para cada $n \in \mathbb{N}$, seja $q_{n}: \ell_{\infty} \rightarrow \mathbb{K}$ o funcional linear e contínuo definido por $q_{n}(\lambda)=n \lambda_{n}$, para cada $\lambda=\left(\lambda_{j}\right)_{j} \in \ell_{\infty}$. Para $\lambda=(1,1, \ldots), \sum\left|q_{n}(\lambda)\right|=\sum n=\infty$. Logo a série $\sum q_{n}$ não é $\omega^{*}$ incondicionalmente convergente em $\left(\ell_{\infty}\right)^{\prime}$.

Proposição 1.3.8. Sejam E um espaço de Banach e $\sum g_{n}$ uma série incondicionalmente convergente em $E^{\prime}$. Então $\sum g_{n}$ é $\omega^{*}$ incondicionalmente convergente.

Demonstração: Seja $\sum g_{n}$ uma série incondicionalmente convergente em $E^{\prime}$. Dado $x \in E$, se $x=0$, então é imediato que $\sum g_{n}(x)$ é incondicionalmente convergente em $\mathbb{K}$. Se $x \neq 0$, então pela proposição 1.3.2, existe $g$ em $E^{\prime}$ tal que dado $\epsilon>0$, existe $F_{\epsilon}$ (finito) $\subset \mathbb{N}$ tal que para todo $F$ com $F_{\epsilon} \subset F$ (finito) $\subset \mathbb{N}$, temos que $\left\|g-\sum_{n \in F} g_{n}\right\|<\epsilon /\|x\|$. 
Como, $\left\|\left(g-\sum_{n \in F} g_{n}\right)(x)\right\| \leq\left\|g-\sum_{n \in F} g_{n}\right\|\|x\|$, segue que a série numérica $\sum g_{n}(x)$ é somável para $g(x)$, logo, novamente usando a proposição 1.3.2, incondicionalmente convergente em $\mathbb{K}$.

Usaremos o seguinte lema na demonstração de alguns resultados no capítulo 2 .

Lema 1.3.9. Seja $\sum \mu_{n}$ uma série de escalares. Se a série $\sum\left|\mu_{n}\right|$ diverge, então existe uma seqüência não-crescente $\lambda_{1} \geq \lambda_{2} \geq \cdots$ de números positivos convergindo a zero tal que a série $\sum\left|\lambda_{n} \mu_{n}\right|$ também diverge.

Demonstração: Como $\sum\left|\mu_{n}\right|$ diverge, existe $N_{1}, N_{2}, \ldots$ uma seqüência crescente de inteiros positivos tal que

$$
\left|\mu_{N_{k}}\right|+\cdots+\left|\mu_{N_{k+1}-1}\right|>4^{k} \text {, para cada } k \in \mathbb{N} \text {. }
$$

Considere $\lambda_{n}=2^{-k}$ para $N_{k} \leq n<N_{k+1}$. Então $\left(\lambda_{n}\right)_{n}$ é uma seqüência não crescente de inteiros positivos que converge para zero. Como

$$
\begin{gathered}
\left|\lambda_{N_{K}} \mu_{N_{k}}\right|+\cdots+\left|\lambda_{N_{k+1}-1} \mu_{N_{k+1}-1}\right|= \\
=\left|\lambda_{N_{K}}\right|\left|\mu_{N_{k}}\right|+\cdots+\left|\lambda_{N_{k+1}-1}\right|\left|\mu_{N_{k+1}-1}\right|= \\
=2^{-k}\left(\left|\mu_{N_{k}}\right|+\cdots+\left|\mu_{N_{k+1}-1}\right|\right)>2^{-k} 4^{k}=2^{k},
\end{gathered}
$$

a série $\sum\left|\lambda_{n} \mu_{n}\right|$ diverge.

A proposição que apresentamos em seguida está demonstrada em McArthur and Retherford [15] e será utilizada na demonstração de resultados no capítulo 2.

Proposição 1.3.10. Sejam $E$ um espaço de Banach, $\sigma$ um conjunto finito de inteiros positivos, $\left(x_{j}\right)_{j \in \sigma}$ uma familia de elementos de $E,\left(t_{j}\right)_{j \in \sigma}$ uma família de números complexos. Então vale a seguinte desigualdade:

$$
\left\|\sum_{j \in \sigma} t_{j} x_{j}\right\| \leq 4 \sup _{j \in \sigma}\left|t_{j}\right| \sup _{\sigma^{\prime} \subseteq \sigma}\left\|\sum_{j \in \sigma^{\prime}} x_{j}\right\| .
$$


Demonstração: Como $\sigma$ é um conjunto finito, podemos assumir que $\sigma=\{1, \ldots, n\}$. Para demonstrar a desigualdade vamos proceder da seguinte maneira:

1) Vamos assumir que todos os escalares são números reais não negativos com $t_{1} \geq$ $t_{2} \geq \cdots \geq t_{n}$. Então

$$
\begin{gathered}
\left\|\sum_{j=1}^{n} t_{j} x_{j}\right\|=\left\|\sum_{j=1}^{n-1}\left(t_{j}-t_{j+1}\right)\left(x_{1}+\cdots+x_{j}\right)+t_{n}\left(x_{1}+\cdots+x_{n}\right)\right\| \leq \\
\leq \sum_{j=1}^{n-1}\left(t_{j}-t_{j+1}\right)\left\|x_{1}+\cdots+x_{j}\right\|+t_{n}\left\|x_{1}+\cdots+x_{n}\right\| \leq \\
\leq\left[\sum_{j=1}^{n-1}\left(t_{j}-t_{j+1}\right)+t_{n}\right] \sup _{\sigma^{\prime} \subseteq \sigma}\left\|\sum_{j \in \sigma^{\prime}} x_{j}\right\| \leq \\
\leq \sup _{j \varepsilon \sigma}\left|t_{j}\right| \sup _{\sigma^{\prime} \subseteq \sigma}\left\|\sum_{j \in \sigma^{\prime}} x_{j}\right\|
\end{gathered}
$$

2) Em seguida vamos assumir que todos os escalares são números reais negativos com $\left|t_{1}\right| \geq\left|t_{2}\right| \geq \cdots \geq\left|t_{n}\right|$. Então

$$
\left\|\sum_{j=1}^{n} t_{j} x_{j}\right\|=\left\|\sum_{j=1}^{n}-\left|t_{j}\right| x_{j}\right\|=\left\|\sum_{j=1}^{n}\left|t_{j}\right| x_{j}\right\| .
$$

Agora por 1) temos que

$$
\left\|\sum_{j=1}^{n} t_{j} x_{j}\right\| \leq \sup _{j \in \sigma}\left|t_{j}\right| \sup _{\sigma^{\prime} \subseteq \sigma}\left\|\sum_{j \in \sigma^{\prime}} x_{j}\right\|
$$

Agora, para escalares reais quaisquer aplicamos isto para escalares positivos e negativos separadamente. Se $t_{j} \geq 0$ para $j=1, \ldots, m ; t_{j}<0$ para $j=m+1, \ldots, n ; t_{1} \geq t_{2} \geq$ $\cdots \geq t_{m}$ e $\left|t_{m+1}\right| \geq\left|t_{m+2}\right| \geq \cdots \geq\left|t_{n}\right|$, então por 1) e 2) temos que

$$
\begin{gathered}
\left\|\sum_{j=1}^{n} t_{j} x_{j}\right\|=\left\|\sum_{j=1}^{m} t_{j} x_{j}+\sum_{j=m+1}^{n} t_{j} x_{j}\right\| \leq\left\|\sum_{j=1}^{m} t_{j} x_{j}\right\|+\left\|\sum_{j=m+1}^{n} t_{j} x_{j}\right\| \leq \\
\leq 2 \sup _{j \in \sigma}\left|t_{j}\right| \sup _{\sigma^{\prime} \subseteq \sigma}\left\|\sum_{j \in \sigma^{\prime}} x_{j}\right\|
\end{gathered}
$$


Então para $t_{j}=a_{j}+\imath b_{j} \in \mathscr{C}$, escrevemos $\sum t_{j} x_{j}$ como $\sum a_{j} x_{j}+i \sum b_{j} x_{j}$. Aplicando a desigualdade para escalares reais para cada soma separadamente obtemos o resultado.

$$
\begin{gathered}
\left\|\sum_{j=1}^{n} t_{j} x_{j}\right\|=\left\|\sum_{j=1}^{n} a_{j} x_{j}+\sum_{j=1}^{n} \imath b_{j} x_{j}\right\| \leq\left\|\sum_{j=1}^{n} a_{j} x_{j}\right\|+|\imath|\left\|\sum_{j=1}^{n} b_{j} x_{j}\right\| \leq \\
4 \sup _{j \in \sigma}\left|t_{j}\right| \sup _{\sigma^{\prime} \subseteq \sigma}\left\|\sum_{j \in \sigma^{\prime}} x_{j}\right\|
\end{gathered}
$$

Utilizamos alguns resultados envolvendo bases de Schauder nos capítulos 2 e 3 desta dissertação. Apresentamos a seguir as definições e resultados que utilizaremos.

Definições 1.3.11. (Bases de Schauder)

Sejam E um espaço de Banach e $\left(x_{n}\right)_{n}$ uma seqüência em $E$.

1. Dizemos que $\left(x_{n}\right)_{n}$ é uma base de Schauder para $E$ se para cada $x$ em E existe uma única seqüência de escalares $\left(\alpha_{n}\right)_{n}$ tal que a série $\sum \alpha_{n} x_{n}$ converge para $x$ em $E$.

2. Dizemos que $\left(x_{n}\right)_{n}$ é uma seqüência básica de Schauder em $E$ se $\left(x_{n}\right)_{n}$ é uma base de Schauder para o subespaço $\overline{\left[x_{n}: n \in \mathbb{I N}\right]}$.

3. Dizemos que uma base de Schauder $\left(x_{n}\right)_{n}$ para E é normalizada se $\left\|x_{n}\right\|=1$, para todo $n \in \mathbb{N}$.

4. Dizemos que uma base de Schauder $\left(x_{n}\right)_{n}$ para $E$ é incondicional se para cada $x$ em E a expansão $x=\sum \alpha_{n} x_{n}$ é uma série incondicionalmente convergente.

Neste trabalho, uma seqüência $\left(x_{n}\right)_{n} \subset E$ será chamada de base (seqüência básica) quando ela for uma base de Schauder (seqüência básica de Schauder) para $E$.

Exemplo 1.3.12. Se E é $c_{0}$ ou $\ell_{p}$, com $1 \leq p<\infty$, considere $\left(e_{n}\right)_{n}$ a seqüência de vetores unitários de $E$, então $\left(e_{n}\right)_{n}$ é uma base de Schauder normalizada incondicional para E. Esta seqüência será chamada de base canônica de $E$.

Definição 1.3.13. Seja E um espaço de Banach com base de Schauder $\left(x_{n}\right)_{n}$. Para cada $n \in \mathbb{N}$, definimos o m-ésimo coeficiente funcional $x_{m}^{\prime}$ associado a $\left(x_{n}\right)_{n}$ como a aplicação:

$$
\begin{gathered}
x_{m}^{\prime}: E \rightarrow \mathbb{K} \text { dada por : } \\
x_{m}^{\prime}\left(\sum_{n=1}^{\infty} \alpha_{n} x_{n}\right)=\alpha_{m}, \quad \text { para cada } x=\sum_{n=1}^{\infty} \alpha_{n} x_{n} \text { em } E .
\end{gathered}
$$


Também definimos a m-ésima projeção natural $P_{m}$ relativa à base $\left(x_{n}\right)_{n}$ como a aplicação:

$$
\begin{gathered}
P_{m}: E \rightarrow \text { Edada por : } \\
P_{m}\left(\sum_{n=1}^{\infty} \alpha_{n} x_{n}\right)=\sum_{n=1}^{m} \alpha_{n} x_{n}, \quad \text { para cada } x=\sum_{n=1}^{\infty} \alpha_{n} x_{n} \text { em } E .
\end{gathered}
$$

Cada coeficiente funcional é um funcional linear sobre $E$ e cada projeção é um operador linear sobre $E$.

Vamos agora mostrar que os coeficientes funcionais e as projeções naturais são contínuos. Para isto vamos usar outra norma, que não é a norma original de $E$.

Definição 1.3.14. Seja $\left(x_{n}\right)_{n}$ uma base de Schauder para um espaço de Banach E. Definimos uma norma e denotamos $\|\cdot\|_{\left(x_{n}\right)}$ por:

$$
\|x\|_{\left(x_{n}\right)}=\sup _{m}\left\|\sum_{n=1}^{m} \alpha_{n} x_{n}\right\| \text {, para cada } x=\sum_{n=1}^{\infty} \alpha_{n} x_{n} \text { em } E \text {. }
$$

Teorema 1.3.15. Seja $\left(x_{n}\right)_{n}$ uma base de Schauder para um espaço de Banach E. Então $\|$. $\|_{\left(x_{n}\right)}$ é uma norma equivalente à norma original de $E$ e, $\|x\| \leq\|x\|_{\left(x_{n}\right)}$, para todo $x \in E$.

Demonstração: Para cada $x=\sum_{n=1}^{\infty} \alpha_{n} x_{n}$ em $E$,

$$
\begin{gathered}
\|x\|=\left\|\sum_{n=1}^{\infty} \alpha_{n} x_{n}\right\|=\left\|\lim _{j \rightarrow \infty} \sum_{n=1}^{j} \alpha_{n} x_{n}\right\|= \\
=\lim _{j \rightarrow \infty}\left\|\sum_{n=1}^{j} \alpha_{n} x_{n}\right\| \leq \sup _{j}\left\|\sum_{n=1}^{j} \alpha_{n} x_{n}\right\|=\|x\|_{\left(x_{n}\right)} .
\end{gathered}
$$

Portanto, $\|x\| \leq\|x\|_{\left(x_{n}\right)}$, para cada $x \in E$.

Com a desigualdade demonstrada acima, temos que $I d:\left(E,\|\cdot\|_{\left(x_{n}\right)}\right) \rightarrow(E,\|\cdot\|)$ é um operador linear, contínuo e bijetor.

Vamos mostrar que $\left(E,\|\cdot\|_{\left(x_{n}\right)}\right)$ é um espaço de Banach, então pelo teorema da aplicação aberta (teorema 1.1.9), teremos que $\|$. $\|$ e $\|\cdot\|_{\left(x_{n}\right)}$ são equivalentes. 
Seja $\left(y_{j}\right)_{j}=\left(\sum_{n=1}^{\infty} \alpha_{n, j} x_{n}\right)_{j}$ uma seqüência de Cauchy em $E$ com relação à norma $\|\cdot\|_{\left(x_{n}\right)}$. Dados $j_{1}, j_{2}, k \in \mathbb{N}, k \geq 2$, temos:

$$
\begin{gathered}
\left|\alpha_{k, j_{1}}-\alpha_{k, j_{2}}\right|\left\|x_{k}\right\|=\left\|\left(\alpha_{k, j_{1}}-\alpha_{k, j_{2}}\right) x_{k}\right\|=\left\|\sum_{n=1}^{k}\left(\alpha_{n, j_{1}}-\alpha_{n, j_{2}}\right) x_{n}-\sum_{n=1}^{k-1}\left(\alpha_{n, j_{1}}-\alpha_{n, j_{2}}\right) x_{n}\right\| \leq \\
2\left\|\sum_{n=1}^{\infty}\left(\alpha_{n, j_{1}}-\alpha_{n, j_{2}}\right) x_{n}\right\|_{\left(x_{n}\right)} \leq 2\left\|y_{j_{1}}-y_{j_{2}}\right\|_{\left(x_{n}\right)}
\end{gathered}
$$

Para $k=1$ e todos $j_{1}, j_{2} \in \mathbb{N}$,

$\left|\alpha_{1, j_{1}}-\alpha_{1, j_{2}}\right|\left\|x_{1}\right\|=\left\|\left(\alpha_{1, j_{1}}-\alpha_{1, j_{2}}\right) x_{1}\right\| \leq\left\|\sum_{n=1}^{\infty}\left(\alpha_{n, j_{1}}-\alpha_{n, j_{2}}\right) x_{n}\right\|_{\left(x_{n}\right)}=\left\|y_{j_{1}}-y_{j_{2}}\right\|_{\left(x_{n}\right)}$

Assim fixado $k \in \mathbb{N}$, segue que $\left(\alpha_{k, j}\right)_{j} \subset \mathbb{K}$ é uma seqüência de Cauchy em $\mathbb{K}$, sendo portanto convergente para, digamos, $\alpha_{k} \in \mathbb{K}$. Consideremos então a seqüência de escalares $\left(\alpha_{n}\right)_{n}$. Vamos mostrar que $\sum_{n=1}^{\infty} \alpha_{n} x_{n}$ converge em $E$ e $y=\sum_{n=1}^{\infty} \alpha_{n} x_{n}$ será nosso candidado a limite de $\left(y_{j}\right)_{j}$ na norma $\|\cdot\|_{\left(x_{n}\right)}$.

Seja $\epsilon>0$. Então existe $j_{\epsilon} \in \mathbb{N}$ tal que $j, j^{\prime} \geq j_{\epsilon} \Rightarrow\left\|y_{j}-y_{j^{\prime}}\right\|_{\left(x_{n}\right)}<\epsilon / 3 \mathrm{e}$

$$
\left\|\sum_{n=1}^{m} \alpha_{n, j} x_{n}-\sum_{n=1}^{m} \alpha_{n, j^{\prime}} x_{n}\right\| \leq\left\|y_{j}-y_{j^{\prime}}\right\|_{\left(x_{n}\right)}<\epsilon / 3, \forall m \in \mathbb{N}, \text { se } j, j^{\prime} \geq j_{\epsilon} .
$$

Fixado $j=j_{\epsilon}$ e fazendo $j^{\prime} \rightarrow \infty$, temos que para cada $m \in \mathbb{N}$ :

$$
\left\|\sum_{n=1}^{m} \alpha_{n, j_{\epsilon}} x_{n}-\sum_{n=1}^{m} \alpha_{n} x_{n}\right\| \leq \epsilon / 3 \text {. }
$$

Seja $m_{\epsilon} \in \mathbb{N}$ tal que $m_{2} \geq m_{1}>m_{\epsilon} \Rightarrow\left\|\sum_{n=m_{1}}^{m_{2}} \alpha_{n, j_{\epsilon}} x_{n}\right\|<\epsilon / 3$. Se $m_{2} \geq m_{1}>m_{\epsilon}$, temos

$\left\|\sum_{n=m_{1}}^{m_{2}} \alpha_{n} x_{n}-\sum_{n=m_{1}}^{m_{2}} \alpha_{n, j_{\epsilon}} x_{n}\right\| \leq\left\|\sum_{n=1}^{m_{2}} \alpha_{n} x_{n}-\sum_{n=1}^{m_{2}} \alpha_{n, j_{\epsilon}} x_{n}\right\|+\left\|\sum_{n=1}^{m_{1}-1} \alpha_{n} x_{n}-\sum_{n=1}^{m_{1}-1} \alpha_{n, j_{\epsilon}} x_{n}\right\| \leq \frac{2 \epsilon}{3}$.

Logo,

$$
\left\|\sum_{n=m_{1}}^{m_{2}} \alpha_{n} x_{n}\right\| \leq\left\|\sum_{n=m_{1}}^{m_{2}} \alpha_{n} x_{n}-\sum_{n=m_{1}}^{m_{2}} \alpha_{n, j_{\epsilon}} x_{n}\right\|+\left\|\sum_{n=m_{1}}^{m_{2}} \alpha_{n, j_{\epsilon}} x_{n}\right\|<\epsilon .
$$


Portanto $\sum_{n=1}^{\infty} \alpha_{n} x_{n}=y \in E$, pois $(E,\|\cdot\|)$ é um espaço de Banach. Finalmente, de (1.1) temos que para cada $j \geq j_{\epsilon}$

$$
\left\|\sum_{n=1}^{m}\left(\alpha_{n, j}-\alpha_{n}\right) x_{n}\right\| \leq \epsilon / 3 \forall m \in \mathbb{N}
$$

isto é,

$$
\sup _{m}\left\|\sum_{n=1}^{m}\left(\alpha_{n, j}-\alpha_{n}\right) x_{n}\right\| \leq \epsilon / 3<\epsilon .
$$

Logo $y_{j} \stackrel{\|\cdot\|\left(x_{n}\right)}{\rightarrow} y$. Portanto $(E,\|\cdot\|)_{\left(x_{n}\right)}$ é um espaço de Banach.

Proposição 1.3.16. Cada projeção natural $P_{m}$ relativa a uma base de Schauder $\left(x_{n}\right)_{n}$ de um espaço de Banach E é contínua.

Demonstração: Dado $x=\sum_{n=1}^{\infty} \alpha_{n} x_{n}$ em $E$, para cada $m \in \mathrm{N}$ :

$$
\begin{aligned}
\left\|P_{m}(x)\right\|_{\left(x_{n}\right)} & =\left\|P_{m}\left(\sum_{n=1}^{\infty} \alpha_{n} x_{n}\right)\right\|_{\left(x_{n}\right)}=\left\|\sum_{n=1}^{m} \alpha_{n} x_{n}\right\|_{\left(x_{n}\right)} \leq \\
& \leq\left\|\sum_{n=1}^{\infty} \alpha_{n} x_{n}\right\|_{\left(x_{n}\right)}=\|x\|_{\left(x_{n}\right)} .
\end{aligned}
$$

Como as normas $\|\cdot\|$ e $\|\cdot\|_{\left(x_{n}\right)}$ são equivalentes, segue que $P_{m}$ é contínua para cada $m \in \mathbb{N}$.

Corolário 1.3.17. Seja $\left\{P_{m}: m \in \mathbb{N}\right\}$ a coleção de projeção naturais relativas a uma base de Schauder $\left(x_{n}\right)_{n}$ de um espaço de Banach $E$, então $\sup _{n}\left\|P_{n}\right\|<\infty$.

Demonstração: Da demonstração da proposição 1.3.16, temos que $\left\|P_{m}\right\|_{\left(x_{n}\right)} \leq 1$ para cada $m \in \mathbb{N}$. Como as normas $\|\cdot\|$ e $\|\cdot\|_{\left(x_{n}\right)}$ são equivalentes, segue o resultado.

Definição 1.3.18. Seja $\left(x_{n}\right)_{n}$ uma base de Schauder para um espaço de Banach E. Chamamos de constante básica para a base $\left(x_{n}\right)_{n}$ ao número $\sup _{n}\left\|P_{n}\right\|$. Denotaremos a constante básica por $C$.

A constante básica depende da norma do espaço e temos do corolário 1.3.17 que $C<\infty$. 
Proposição 1.3.19. Sejam E um espaço de Banach e $\left(x_{n}\right)_{n}$ uma base de Schauder para $E$ com coeficientes funcionais associados $\left(x_{n}^{\prime}\right)_{n}$. Então os coeficientes funcionais são contínuos.

Demonstração: Para cada $m \in \mathbb{N}$, seja $T_{m}:\left[x_{m}\right] \rightarrow \mathbb{K}$ a aplicação linear contínua definida por $T_{m}\left(\alpha x_{m}\right)=\alpha$. Dado $x=\sum_{n=1}^{\infty} \alpha_{n} x_{n}$ em $E$, se $m=1$, então $x_{1}^{\prime}(x)=\alpha_{1}=$ $\left(T_{1} \circ P_{1}\right)(x)$. Como $T_{1}$ e $P_{1}$ são contínuas, segue que $x_{1}^{\prime}$ é contínuo. Se $m \geq 2$, então

$$
\begin{gathered}
x_{m}^{\prime}(x)=\alpha_{m}=T_{m}\left(\alpha_{m} x_{m}\right)=T_{m}\left(\sum_{n=1}^{m} \alpha_{n} x_{m}-\sum_{n=1}^{m-1} \alpha_{n} x_{m}\right)=T_{m}\left(P_{m}(x)-P_{m-1}(x)\right)= \\
=T_{m}\left(P_{m}-P_{m-1}\right)(x)
\end{gathered}
$$

e como $T_{m}$ e $P_{m}$ são contínuas, segue que $x_{m}^{\prime}$ é contínuo.

Na demonstração de alguns resultados no capítulo 2 utilizaremos algumas normas equivalentes à norma original de $E$.

Definição 1.3.20. Seja $\left(x_{n}\right)_{n}$ uma base de Schauder incondicional para um espaço de Banach E. Então definimos a norma incondicional multiplicador limitado relativa à base $\left(x_{n}\right)_{n}$ por

$$
\|x\|_{(b m u)}=\sup \left\{\left\|\sum_{n=1}^{\infty} \beta_{n} \alpha_{n} x_{n}\right\|:\left(\beta_{n}\right) \in S_{\ell_{\infty}}\right\} \text { para cada } x=\sum \alpha_{n} x_{n} \text { em } E .
$$

Observamos que $\|x\|_{(b m u)}<\infty$ para todo $x \in E$, uma vez que se $\sup \left\{\left\|\sum_{n=1}^{\infty} \beta_{n} \alpha_{n} x_{n}\right\|:\left(\beta_{n}\right) \epsilon S_{\ell_{\infty}}\right\}=\infty$ para um certo $x=\sum_{n=1}^{\infty} \alpha_{n} x_{n} \in E$, então dado $n_{1} \in \mathbb{N}$, é possível obter $n_{2}>n_{1}$ e escalares $\beta_{1}, \ldots, \beta_{n_{2}}$ tais que $\left|\beta_{i}\right| \leq 1$ para $1 \leq i \leq n_{2}$ e $\left\|\sum_{n=1}^{n_{2}} \beta_{n} \alpha_{n} x_{n}\right\| \geq 1+\sum_{n=1}^{n_{1}}\left\|\alpha_{n} x_{n}\right\|$.

Assim segue que $\left\|\sum_{n=n_{1}+1}^{n_{2}} \beta_{n} \alpha_{n} x_{n}\right\|=\left\|\sum_{n=1}^{n_{2}} \beta_{n} \alpha_{n} x_{n}-\sum_{n=1}^{n_{1}} \beta_{n} \alpha_{n} x_{n}\right\| \geq$

$\geq\left\|\sum_{n=1}^{n_{2}} \beta_{n} \alpha_{n} x_{n}\right\|-\left\|\sum_{n=1}^{n_{1}} \beta_{n} \alpha_{n} x_{n}\right\| \geq 1+\sum_{n=1}^{n_{2}}\left\|\alpha_{n} x_{n}\right\|-\left\|\sum_{n=1}^{n_{1}}\right\| \beta_{n} \alpha_{n} x_{n} \|=$

$=1+\sum_{n=1}^{n_{2}}\left\|\alpha_{n} x_{n}\right\|-\sum_{n=1}^{n_{1}}\left|\beta_{n}\right|\left\|\alpha_{n} x_{n}\right\| \geq$

$\geq 1+\sum_{n=1}^{n_{2}}\left\|\alpha_{n} x_{n}\right\|-\sum_{n=1}^{n_{1}}\left\|\alpha_{n} x_{n}\right\|=1$. Seja $\gamma_{n}=\beta_{n}$ para $n=1, \ldots, n_{2}$.

Do mesmo modo é possível obter $n_{3}>n_{2}$ e escalares $\beta_{n_{2}+1}^{\prime}, \ldots, \beta_{n_{3}}^{\prime}$ tais que $\left|\beta_{i}^{\prime}\right| \leq 1$ para $n_{2}+1 \leq i \leq n_{3}$ e $\left\|\sum_{n=1}^{n_{3}} \beta_{n}^{\prime} \alpha_{n} x_{n}\right\| \geq 1+\sum_{n=1}^{n_{2}}\left\|\alpha_{n} x_{n}\right\|$. E assim $\left\|\sum_{n=n_{2}+1}^{n_{3}} \beta_{n}^{\prime} \alpha_{n} x_{n}\right\| \geq 1$. Seja $\gamma_{n}=\beta_{n}^{\prime}$ para $n=n_{2}+1, \ldots, n_{3}$. 
Prosseguindo dessa forma, obtemos indutivamente $n_{1}<n_{2}<n_{3}<\ldots$ e $\left(\gamma_{n}\right)_{n}$ tais que $\left|\gamma_{n}\right| \leq 1$ e $\left\|\sum_{n=n_{i}+1}^{n_{i+1}} \gamma_{n} \alpha_{n} x_{n}\right\| \geq 1$

A seqüência de somas parciais da série $\sum_{n=1}^{\infty} \gamma_{n} \alpha_{n} x_{n}$ não é de Cauchy, portanto a série $\sum_{n=1}^{\infty} \gamma_{n} \alpha_{n} x_{n}$ não converge, uma contradição, pois por hipótese $\left(x_{n}\right)_{n}$ é uma base incondicional para $E$.

Teorema 1.3.21. Seja $\left(x_{n}\right)_{n}$ uma base de Schauder incondicional para um espaço de Banach E. Então $\|\cdot\|_{(b m u)}$ é uma norma equivalente à norma original de $E$, $e$

$$
\|x\| \leq\|x\|_{\left(x_{n}\right)} \leq\|x\|_{(b m u)} \text {, para cada } x \text { em } E .
$$

Demonstração: Seja $x=\sum_{n=1}^{\infty} \alpha_{n} x_{n}$ em $E$. Do teorema 1.3.15, temos que $\|x\| \leq$ $\|x\|_{\left(x_{n}\right)}$.

Dado $m \in \mathbb{N}$, considere $\beta_{n}=1$ para $n=1, \ldots, m$ e $\beta_{n}=0$ para $n>m$. Então, $\left(\beta_{n}\right)_{n} \in S_{\ell_{\infty}} \mathrm{e}$

$$
\left\|\sum_{n=1}^{m} \alpha_{n} x_{n}\right\|=\left\|\sum_{n=1}^{\infty} \beta_{n} \alpha_{n} x_{n}\right\| \leq \sup \left\{\left\|\sum_{n=1}^{\infty} \beta_{n} \alpha_{n} x_{n}\right\|\left(\beta_{n}\right)_{n} \in S_{\ell_{\infty}}\right\} \leq\left\|x_{n}\right\|_{(b m u)}
$$

Logo, $\sup _{m}\left\|\sum_{n=1}^{m} \alpha_{n} x_{n}\right\| \leq\|x\|_{(b m u)}$. E portanto $\|x\|_{\left(x_{n}\right)} \leq\|x\|_{(b m u)}$

Temos que $I d:\left(E,\|\cdot\|_{(b m u)}\right) \rightarrow(E,\|\cdot\|)$ é um operador linear, contínuo e bijetor. Vamos mostrar que $\left(E,\|\cdot\|_{(b m u)}\right)$ é um espaço de Banach, então pelo teorema da aplicação aberta, teorema 1.1.9, teremos que as normas $\|\cdot\| \mathrm{e}\|\cdot\|_{(b m u)}$ são equivalentes.

Seja $\left(y_{j}\right)_{j}=\left(\sum_{n=1}^{\infty} \alpha_{n, j} x_{n}\right)_{j}$ uma seqüência de Cauchy em $E$ com relação à norma $\|$. $\|_{(b m u)}$. Então $\left(y_{j}\right)_{j}$ é também uma seqüência de Cauchy em $(E,\|\cdot\|)$, portanto $y_{j} \stackrel{\|\cdot\|}{\rightarrow} x=\sum_{n=1}^{\infty} \alpha_{n} x_{n} \in E$. Vamos mostrar que $y_{j} \rightarrow x$ em $\left(E,\|\cdot\|_{(b m u)}\right)$.

Seja $\left(x_{n}^{\prime}\right)_{n}$ a seqüência de coeficientes funcionais associada a $\left(x_{n}\right)_{n}$. Para cada $n \in \mathbb{N}$ temos que $x_{n}^{\prime}$ é contínuo, então $x_{n}^{\prime}\left(y_{j}\right) \stackrel{j}{\rightarrow} x_{n}^{\prime}(x)$, logo $\alpha_{n j} \rightarrow \alpha_{n}$. Seja $C$ a constante básica de $\left(x_{n}\right)_{n}$. Dado $\epsilon>0$ é possível obter $j_{\epsilon} \in \mathbb{I N}$ tal que $j, j^{\prime} \geq j_{\epsilon} \Rightarrow \| \sum_{n=1}^{\infty} \alpha_{n, j^{\prime}} x_{n}-$ $\sum_{n=1}^{\infty} \alpha_{n, j} x_{n} \|_{(b m u)} \leq \epsilon / C$.

Dados $m \in \mathbb{N},\left(\beta_{n}\right)_{n} \in S_{\ell_{\infty}}$ e $j, j^{\prime} \geq j_{\epsilon}$, temos que

$$
\left\|\sum_{n=1}^{m} \beta_{n} \alpha_{n, j^{\prime}} x_{n}-\sum_{n=1}^{m} \beta_{n} \alpha_{n, j} x_{n}\right\| \leq C\left\|\sum_{n=1}^{\infty} \beta_{n} \alpha_{n, j^{\prime}} x_{n}-\sum_{n=1}^{\infty} \beta_{n} \alpha_{n, j} x_{n}\right\| \leq
$$




$$
\leq C\left\|\sum_{n=1}^{\infty} \alpha_{n, j^{\prime}} x_{n}-\sum_{n=1}^{\infty} \alpha_{n, j} x_{n}\right\|_{(b m u)} \leq \frac{C \epsilon}{C}=\epsilon .
$$

Assim $\left\|\sum_{n=1}^{m} \beta_{n} \alpha_{n} x_{n}-\sum_{n=1}^{m} \beta_{n} \alpha_{n, j} x_{n}\right\| \leq \epsilon$ para todo $m \in \mathbb{N}, \forall \beta_{n} \in S_{\ell_{\infty}}, \forall j \geq j_{\epsilon}$.

Fazendo $m \rightarrow \infty$, temos que $\left\|\sum_{n=1}^{\infty} \beta_{n} \alpha_{n} x_{n}-\sum_{n=1}^{\infty} \beta_{n} \alpha_{n, j} x_{n}\right\| \leq \epsilon, \forall \beta_{n} \in S_{\ell_{\infty}}, \forall j \geq$ $j_{\epsilon}$. Então para todo $j \geq j_{\epsilon}$, temos que $\left\|\sum_{n=1}^{\infty} \alpha_{n} x_{n}-\sum_{n=1}^{\infty} \alpha_{n, j} x_{n}\right\|_{(b m u)} \leq \epsilon$, ou seja, $\left\|x-y_{j}\right\|_{(b m u)} \leq \epsilon . A \operatorname{ssim}\left(E,\|\cdot\|_{(b m u)}\right)$ é um espaço de Banach.

Definição 1.3.22. Seja $\left(x_{n}\right)_{n}$ uma base de Schauder incondicional para um espaço de Banach E. Então definimos a norma incondicional relativa à base $\left(x_{n}\right)_{n}$, e escrevemos $\|\cdot\|_{(u)}$, por

$$
\|x\|_{(u)}=\sup \left\{\left\|\sum_{n \in A} \alpha_{n} x_{n}\right\|: A \text { é um subconjunto finito de } \mathbb{I N}\right\}
$$

para cada $x=\sum \alpha_{n} x_{n}$ em $E$.

Teorema 1.3.23. Seja $\left(x_{n}\right)_{n}$ uma base de Schauder incondicional para um espaço de Banach E. Então $\|\cdot\|_{u}$ é uma norma equivalente à norma original de $E$, $e$

$$
\|x\| \leq\|x\|_{\left(x_{n}\right)} \leq\|x\|_{(u)} \leq\|x\|_{(b m u)} \text {, para cada } x \text { em } E .
$$

Demonstração: Vamos mostrar que $\|x\| \leq\|x\|_{\left(x_{n}\right)} \leq\|x\|_{(u)} \leq\|x\|_{(b m u)}$, para cada $x$ em $E$. Do teorema 1.3.15, temos que $\|x\| \leq\|x\|_{\left(x_{n}\right)}$, para cada $x$ em $E$.

Para cada $x=\sum \alpha_{n} x_{n}$ em $E$ temos que:

para cada $m \in \mathbb{N},\{1, \ldots, m\}$ é um subconjunto finito de $\mathbb{N}$, então,

$$
\left\|\sum_{n=1}^{m} \alpha_{n} x_{n}\right\| \leq\left\|\sum_{n=1}^{\infty} \alpha_{n} x_{n}\right\|_{(u)}
$$

Logo,

$$
\sup _{m}\left\|\sum_{n=1}^{m} \alpha_{n} x_{n}\right\| \leq\left\|\sum_{n=1}^{\infty} \alpha_{n} x_{n}\right\|_{(u)} .
$$

E portanto $\|x\|_{\left(x_{n}\right)} \leq\|x\|_{(u)}$.

Para cada subconjunto finito $A \subset \mathbb{N}$, considere $\beta_{n}=1$ para $n \in A$ e $\beta_{n}=0$ para $n \notin A$. Então, $\left(\beta_{n}\right)_{n} \in S_{\ell_{\infty}} \mathrm{e}$ 


$$
\left\|\sum_{n \in A} \alpha_{n} x_{n}\right\|=\left\|\sum_{n=1}^{\infty} \beta_{n} \alpha_{n} x_{n}\right\| \leq\left\|\sum_{n=1}^{\infty} \alpha_{n} x_{n}\right\|_{(b m u)}
$$

Logo,

$\sup \left\{\left\|\sum_{n \in A} \alpha_{n} x_{n}\right\|:\right.$ A é um subconjunto finito de $\left.\mathbb{N}\right\}=\left\|\sum_{n=1}^{\infty} \alpha_{n} x_{n}\right\|_{(u)} \leq\left\|\sum_{n=1}^{\infty} \alpha_{n} x_{n}\right\|_{(b m u)}$.

E portanto $\|x\|_{(u)} \leq\|x\|_{(b m u)}$.

Assim temos que $\|x\| \leq\|x\|_{\left(x_{n}\right)} \leq\|x\|_{(u)} \leq\|x\|_{(b m u)}$, para cada $x \in E$.

Segue do teorema 1.3.21 que a norma $\|x\|_{(u)}$ é equivalente à norma original de $E$.

Definição 1.3.24. (Bases equivalentes)

Sejam E, F espaços de Banach, $e\left(x_{n}\right)_{n},\left(y_{n}\right)_{n}$ bases de Schauder de $E$ e $F$, respectivamente. As bases $\left(x_{n}\right)_{n} e\left(y_{n}\right)_{n}$ são ditas equivalentes se, para toda seqüência $\left(\alpha_{n}\right)_{n}$ de escalares a série $\sum \alpha_{n} x_{n}$ converge em $E$ se, e somente se, a série $\sum \alpha_{n} y_{n}$ converge em $F$.

A equivalência entre as bases garante isomorfismo entre os respectivos espaços de Banach.

Proposição 1.3.25. Sejam E e F espaços de Banach, $e\left(x_{n}\right)_{n} e\left(y_{n}\right)_{n}$ bases de Schauder de $E$ e $F$, respectivamente. Então as bases $\left(x_{n}\right)_{n} e\left(y_{n}\right)_{n}$ são equivalentes se, e somente se, existe um isomorfismo $T: E \rightarrow F$ tal que $T\left(x_{n}\right)=y_{n}$ para cada $n \in \mathbb{N}$.

Demonstração: É claro que se existe um isomorfismo $T: E \rightarrow F$ tal que $T\left(x_{n}\right)=y_{n}$ para cada $n \in \mathbb{N}$, então $\left(x_{n}\right)_{n}$ e $\left(y_{n}\right)_{n}$ são equivalentes.

Reciprocamente, suponhamos que $\left(x_{n}\right)_{n}$ e $\left(y_{n}\right)_{n}$ são equivalentes. Seja $T: E \rightarrow F$ um operador definido por $T\left(\sum_{n=1}^{\infty} \alpha_{n} x_{n}\right)=\sum_{n=1}^{\infty} \alpha_{n} y_{n}$ para cada $x=\sum_{n=1}^{\infty} \alpha_{n} x_{n}$ em $E$. Como $\left(x_{n}\right)_{n}$ e $\left(y_{n}\right)_{n}$ são equivalentes, é imediato que: $T$ está bem definida, é linear, bijetora e $T\left(x_{n}\right)=y_{n}$, para todo $n \in \mathbb{N}$.

Vamos mostrar que $T$ é contínua. Consideremos $G_{T}=\{(x, T x), x \in E\} \subset E \times F$. Seja $\left(a_{k}, b_{k}\right) \subset G_{T}$ tal que $\left(a_{k}, b_{k}\right) \rightarrow(a, b) \in E \times F$ quando $k \rightarrow \infty$. Então,

$$
a_{k}=\sum_{n=1}^{\infty} \alpha_{n}^{(k)} x_{n} \rightarrow a=\sum_{n=1}^{\infty} \alpha_{n} x_{n} \text { em } E, \text { quando } k \rightarrow \infty e
$$




$$
b_{k}=T\left(a_{k}\right)=\sum_{n=1}^{\infty} \alpha_{n}^{(k)} y_{n} \rightarrow=\sum_{n=1}^{\infty} \beta_{n} y_{n}=b \text { em } F \text {, quando } k \rightarrow \infty
$$

Para cada $n \in \mathbb{N}$, o coeficiente funcional $x_{n}^{\prime}$ é contínuo, $x_{n}^{\prime}\left(a_{k}\right)=\alpha_{n}^{(k)} \rightarrow \alpha_{n}=$ $x_{n}^{\prime}(a)$, quando $k \rightarrow \infty$, e como $y_{n}^{\prime}$ é contínuo, $y_{n}^{\prime}\left(b_{k}\right)=\alpha_{n}^{(k)} \rightarrow \beta_{n}=y_{n}^{\prime}(b)$, quando $k \rightarrow \infty$. Pela unicidade do limite, $\alpha_{n}=\beta_{n}$, para todo $n \in \mathbb{N}$. Logo $b=\sum_{n=1}^{\infty} \alpha_{n} y_{n}=$ $T(a)$ e $(a, b) \in G_{T}$.

Segue do Teorema do Gráfico Fechado (teorema 1.1.10), que $T$ é contínua e do teorema da Aplicação Aberta (teorema 1.1.9), que $T$ é um isomorfismo.

Terminamos esta seção apresentando resultados de seqüências básicas e seqüências de blocos que utilizaremos no capítulo 3 .

Teorema 1.3.26. Sejam E um espaço de Banach e $\left(x_{n}\right)_{n}$ uma seqüência em E. Se $\left(x_{n}\right)_{n}$ converge fracamente a zero mas não converge a zero em norma, então $\left(x_{n}\right)_{n}$ admite uma subseqüência que é uma seqüência básica.

Demonstração: Ver por exemplo Megginson [16], pág. 364, teorema 4.1.32.

Definição 1.3.27. Sejam E um espaço de Banach, $\left(x_{n}\right)_{n}$ uma base de Schauder para E $e\left(y_{n}\right)_{n}$ uma seqüência de vetores não nulos de E. Dizemos que $\left(y_{n}\right)_{n}$ é uma seqüência de blocos de $\left(x_{n}\right)_{n}$ se cada $y_{n}$ pode ser escrito na forma

$$
y_{n}=\sum_{k=p_{n}}^{q_{n}} \alpha_{k} x_{k}
$$

com $p_{1} \leq q_{1}<p_{2} \leq q_{2}<p_{3} \leq q_{3}<\ldots e\left(\alpha_{k}\right)_{k}$ uma seqüência de escalares.

Teorema 1.3.28. Sejam $E=c_{0}$ ou $\ell_{p}$, com $1 \leq p<\infty e\left(e_{n}\right)_{n}$ a base canônica de $E$. Se $\left(x_{n}\right)_{n}$ é uma seqüência de blocos de $\left(e_{n}\right)_{n}$, então $\left(\left\|x_{n}\right\|^{-1} x_{n}\right)_{n}$ é uma seqüência básica equivalente $\grave{a}\left(e_{n}\right)_{n} e \overline{\left[x_{n}: n \in \mathbb{I N}\right]}$ é isomorfo a $E$.

Demonstração: Ver por exemplo Lindenstrauss, Tzafriri [14], pág. 53, teorema 2.a.1.

Teorema 1.3.29 (Princípio de Seleção de Bessaga-Pelczynski). Sejam E um espaço de Banach, $\left(x_{n}\right)_{n}$ uma base de Schauder para $E$ e $\left(x_{n}^{\prime}\right)_{n}$ a seqüência de coeficientes 
funcionais associada à $\left(x_{n}\right)_{n}$. Seja $\left(y_{n}\right)_{n}$ uma seqüência em E tal que $\lim _{m} x_{n}^{\prime} y_{m}=0$ para cada $n$, mas $\left(y_{n}\right)_{n}$ não converge a zero em norma. Então existe uma subseqüência de $\left(y_{n}\right)_{n}$ que é uma seqüência básica equivalente a uma seqüência de blocos de $\left(x_{n}\right)_{n}$.

Demonstração: Ver por exemplo Megginson [16], pág. 396, teorema 4.3.19.

\subsection{Operadores compactos}

No que segue apresentamos a definição de operadores compactos e resultados sobre os mesmos que utilizaremos no decorrer desta dissertação.

Definição 1.4.1. (Operadores Compactos) Sejam E e F espaços de Banach. Um operador linear $T: E \rightarrow F$ é dito compacto se para todo subconjunto $B$ limitado de $E, T(B)$ é um subconjunto relativamente compacto de $F$.

O conjunto dos operadores compactos de $E$ em $F$ será denotado por $K(E, F)$. No que segue vamos mostrar que $K(E, F)$ é um subconjunto de $L(E, F)$.

Proposição 1.4.2. Sejam E e F espaços de Banach, e $T: E \rightarrow F$ um operador linear compacto. Então $T$ é contínuo.

Demonstração: Se $T$ é compacto, então $T\left(B_{E}\right)$ é relativamente compacto, logo $\overline{T\left(B_{E}\right)}$ é um subconjunto limitado em $F$. Assim $\sup \{\|T x\|:\|x\|=1\}<\infty$ e conseqüentemente $T$ é contínuo.

Não é difícil mostrar que $K(E, F)$ é um subespaço de $L(E, F)$.

Proposição 1.4.3. Se E é um espaço de Banach, então o operador identidade definido em $E$ é compacto se, e somente se, $E$ tem dimensão finita.

Demonstração: Sabemos que $B_{E}$ é compacta se, e somente se, $\operatorname{dim} E<\infty$. Assim, $\operatorname{Id}\left(B_{E}\right)=B_{E}$ é relativamente compacto se, e somente se, $\operatorname{dim} E<\infty$. Logo, o operador identidade definido em $E$ é compacto se, e somente se, $E$ tem dimensão finita.

Proposição 1.4.4. Sejam $E$ e $F$ espaços de Banach e $T: E \rightarrow F$ um operador linear de posto finito. Então $T$ é compacto se, e somente se, é contínuo. 
Demonstração: Se $T$ é compacto então pela proposição 1.4 .2 segue que $T$ é contínuo. Agora, se $T$ é contínuo, então $T\left(B_{E}\right)$ é um subconjunto limitado de $T(E)$. Agora, como $T$ tem posto finito, $\operatorname{dim} T(E)<\infty, \log o T\left(B_{E}\right)$ é relativamente compacto e conseqüentemente $T$ é compacto.

Lembramos que um subconjunto $S$ de um espaço métrico é totalmente limitado se para todo $\epsilon>0$ é possível cobrir $S$ por um número finito de bolas abertas de raio $\epsilon$ e centro em $S$. Lembramos também que um subconjunto $S$ de um espaço topológico $X$ é seqüencialmente compacto se toda seqüência em $S$ admite uma subseqüência que converge para um ponto de $X$. Sabemos da teoria de espaços métricos que um subconjunto de um espaço métrico completo é relativamente compacto se, e somente se, é totalmente limitado se, e somente se, é seqüencialmente compacto. (Por exemplo, ver Dunford e Schwartz [8], pag. 22, teorema 15). Com isto temos a seguinte caracterização de operadores compactos.

Proposição 1.4.5. Sejam E e F espaços de Banach, e $T: E \rightarrow F$ um operador linear. Então são equivalentes:

(a) $T$ é compacto.

(b) $T\left(B_{E}\right)$ é um subconjunto relativamente compacto de $F$.

(c) Para todo subconjunto $B$ limitado de $E, T(B)$ é um subconjunto totalmente limitado de $F$.

(d) Toda seqüência limitada $\left(x_{n}\right)_{n}$ em E admite uma subseqüência $\left(x_{n_{j}}\right)_{j}$ tal que $\left(T x_{n_{j}}\right)_{j}$ é convergente.

Se $E$ e $F$ são espaços de Banach então $K(E, F)$ é um subespaço fechado de $L(E, F)$, como mostra a seguinte proposição.

Proposição 1.4.6. Sejam E e F espaços de Banach, e $\left(T_{n}\right)_{n}$ uma seqüência de operadores lineares compactos de $E$ em $F$ que converge para algum $T$ em $L(E, F)$. Então $T$ é compacto.

Demonstração: Suponhamos que $\left(T_{n}\right)_{n}$ é uma seqüência de operadores lineares compactos de $E$ em $F$ que converge para algum $T$ em $L(E, F)$. Dado $\epsilon>0$ existe $n \in \mathbb{N}$ tal que $\left\|T_{n}-T\right\|<\epsilon / 3$. Como $T_{n}$ é compacto, existem $x_{1}, \ldots, x_{m} \in B_{E}$ tais que dado $x \in B_{E}$, existe $x_{i}$ tal que $\left\|T_{n} x-T_{n} x_{i}\right\|<\epsilon / 3$. Segue que dado $x$ em $B_{E}$ existe $x_{i}$ tal que 
$\left\|T x-T x_{i}\right\| \leq\left\|T x-T_{n} x\right\|+\left\|T_{n} x-T_{n} x_{i}\right\|+\left\|T_{n} x_{i}-T x_{i}\right\|<\epsilon / 3+\epsilon / 3+\epsilon / 3=\epsilon$. Portanto $T\left(B_{E}\right)$ é totalmente limitado e logo $T$ é compacto.

Exemplo 1.4.7. O operador linear $T: \ell_{2} \rightarrow \ell_{2}$ definido por $T(\xi)=\left(\xi_{1}, \xi_{2} / 2, \ldots, \xi_{n} / n, \ldots\right)$, para cada $\xi=\left(\xi_{j}\right)_{j}$ em $\ell_{2}$, é compacto.

Para demonstrar isto, para cada $n \in \mathbb{N}$, seja $T_{n}: \ell_{2} \rightarrow \ell_{2}$ um operador linear dado por $T_{n}(\xi)=\left(\xi_{1}, \xi_{2} / 2, \ldots, \xi_{n} / n, 0,0, \ldots\right)$ para cada $\xi=\left(\xi_{j}\right)_{j}$ em $\ell_{2}$.

Para cada $n, T_{n}$ é contínuo e de posto finito, logo compacto. Além disto, para cada $\xi=\left(\xi_{j}\right)_{j} \epsilon \ell_{2}$

$$
\begin{aligned}
\|(T & \left.-T_{n}\right)(\xi) \|^{2}=\sum_{j=n+1}^{\infty}\left|\xi_{j} / j\right|^{2}=\sum_{j=n+1}^{\infty} 1 / j^{2}\left|\xi_{j}\right|^{2} \leq \\
& \leq \frac{1}{(n+1)^{2}} \sum_{j=n+1}^{\infty}\left|\xi_{j}\right|^{2} \leq\|\xi\|^{2} /(n+1)^{2}
\end{aligned}
$$

Logo

$$
\left\|T-T_{n}\right\|=\sup \left\{\left\|\left(T-T_{n}\right)(\xi)\right\|:\|\xi\| \leq 1\right\} \leq 1 /(n+1)
$$

Assim $T_{n} \rightarrow T$, e portanto $T$ é compacto.

Observação 1.4.8. Observemos que a proposição anterior não é verdadeira para convergência pontual. Por exemplo, para cada $n \in \mathbb{N}$, seja $T_{n}: \ell_{2} \rightarrow \ell_{2}$ o operador linear dado por $T_{n}(\xi)=\left(\xi_{1}, \xi_{2}, \ldots, \xi_{n}, 0,0, \ldots\right)$ para cada $\xi=\left(\xi_{j}\right)_{j}$ em $\ell_{2}$.

Para cada $n, T_{n}$ é contínuo e de posto finito, logo compacto. Além disto, é claro que $T_{n}(x) \rightarrow I d(x)$, mas $I d: \ell_{2} \rightarrow \ell_{2}$ não é compacto pois dimensão $\left(\ell_{2}\right)=\infty$.

A seguinte caracterização é um dos resultados importantes da teoria de operadores compactos que será utilizada nos capítulos 2 e 3 . Apresentamos aqui a demonstração que encontra-se em Goldberg [10].

Teorema 1.4.9 (Teorema de Schauder). Um operador linear continuo $T: E \rightarrow F$ entre espaços de Banach é compacto se, e somente se, seu adjunto $T^{*}$ é compacto.

Demonstração: Se T é compacto, então dado $\epsilon>0$, existem $x_{1}, \ldots, x_{n} \in B_{E}$ tais que para cada $x \in B_{E}$ existe $x_{i}$ tal que

$$
\left\|T x-T x_{i}\right\|<\epsilon / 3 .
$$


Seja $A: F^{\prime} \rightarrow \mathbb{K}^{n}$ dado por:

$$
A y^{\prime}=\left(y^{\prime}\left(T x_{1}\right), \ldots, y^{\prime}\left(T x_{n}\right)\right) .
$$

$A$ é linear, contínuo em $\mathbb{K}^{n}$. Portanto $A$ é compacto. Logo existem $y_{1}^{\prime}, \ldots, y_{m}^{\prime} \in B_{F^{\prime}}$ tal que para cada $y^{\prime} \in B_{F^{\prime}}$ existe $y_{j}^{\prime}$ tal que

$$
\left\|A y^{\prime}-A y_{j}^{\prime}\right\|<\epsilon / 3 \text {. }
$$

Mas como

$$
\begin{gathered}
\left\|A y^{\prime}-A y_{j}^{\prime}\right\|=\left\|A\left(y^{\prime}-y_{j}^{\prime}\right)\right\|=\left\|\left(y^{\prime}-y_{j}^{\prime}\right)\left(T x_{1}\right), \ldots,\left(y^{\prime}-y_{j}^{\prime}\right)\left(T x_{n}\right)\right\|= \\
=\left(\sum_{i=1}^{n}\left|\left(y^{\prime}-y_{j}^{\prime}\right)\left(T x_{n}\right)\right|^{2}\right)^{1 / 2}
\end{gathered}
$$

em particular

$$
\left|y^{\prime}\left(T x_{i}\right)-y_{j}^{\prime}\left(T x_{i}\right)\right|<\epsilon / 3, \text { para } 1 \leq i \leq n .
$$

De 1.2 e 1.3 , e observando-se que $y^{\prime}$ e $y_{j}^{\prime} \in B_{F^{\prime}}$, temos que para cada $x \in B_{E}$ e para cada $y^{\prime} \in B_{F^{\prime}}$

$$
\begin{aligned}
& \left|T^{*} y^{\prime} x-T^{*} y_{j}^{\prime} x\right| \leq\left|y^{\prime} T x-y^{\prime} T x_{i}\right|+\left|y^{\prime} T x_{i}-y_{j}^{\prime} T x_{i}\right|+\left|y_{j}^{\prime} T x_{i}-y_{j}^{\prime} T x\right| \leq \\
& \leq\left|y^{\prime}\left(T x-T x_{i}\right)\right|+\left|y^{\prime}\left(T x_{i}\right)-y_{j}^{\prime}\left(T x_{i}\right)\right|+\left|y_{j}^{\prime}\left(T x_{i}-T x\right)\right| \leq \\
& \leq\left\|y^{\prime}\right\|\left\|T x-T x_{i}\right\|+\epsilon / 3+\left\|y_{j}^{\prime}\right\|\left\|T x_{i}-T x\right\|<\epsilon . \\
& \text { Logo }\left\|T^{*} y^{\prime}-T^{*} y_{j}^{\prime}\right\| \leq \epsilon \text { e portanto } T^{*} \text { é compacto. }
\end{aligned}
$$

Por outro lado, se $T^{*}$ é compacto, então pela primeira parte dessa demonstração, $T^{* *}$ é compacto. Sejam $C_{E}: E \rightarrow E^{\prime \prime}$ e $C_{F}: F \rightarrow F^{\prime \prime}$ as inclusões naturais de $E$ em $E^{\prime \prime}$ e $F$ em $F^{\prime \prime}$ respectivamente. Pela proposição $1.1 .8, C_{F}{ }^{-1} T^{* *} C_{E}=T$, como $T^{* *}$ é compacto, $C_{E}$ e $C_{F}^{-1}$ são contínuas, segue que $T$ é compacto. 
A seguir apresentamos dois resultados que encontram-se em Goldberg [10]. Neste, os resultados são enunciados para espaços normados, mas em acordo com o contexto desta dissertação os enunciamos para espaços de Banach. O lema 1.4 .10 será utilizado no demonstração do teorema 1.4.11. Utilizaremos este último para demostrar que alguns operadores que construímos no capítulo 2 são compactos.

Lema 1.4.10. Sejam $S$ um subconjunto limitado de um espaço de Banach $E$ e $x_{1}^{\prime}, \ldots, x_{n}^{\prime} \in$ $E^{\prime}$. Então dado $\epsilon>0$ existem $x_{1}, \ldots, x_{m}$ em $S$ tais que dado $x$ em $S$, podemos escolher $x_{k}$ tal que $\left|x_{i}^{\prime} x-x_{i}^{\prime} x_{k}\right|<\epsilon$, para $1 \leq i \leq n$.

Demonstração: A aplicação $A: E \rightarrow \mathbb{K}^{n}$ definida por $A(x)=\left(x_{1}^{\prime} x, x_{2}^{\prime} x, \ldots, x_{n}^{\prime} x\right)$ é linear e contínua, logo compacta pela proposição 1.4.4. Então existem $x_{1}, x_{2}, \ldots, x_{m}$ em $S$ tais que dado x em $S$ existe $x_{k}$ tal que $\left\|A x-A x_{k}\right\|<\epsilon$.

Como $\left|x_{i}^{\prime} x-x_{i}^{\prime} x_{k}\right| \leq\left\|A x-A x_{k}\right\|$, para $1 \leq i \leq n$, segue o resultado.

Teorema 1.4.11. Sejam $E$ e $F$ espaços de Banach, e $T: E \rightarrow F$ um operador linear contínuo. Se para todo $\epsilon>0$ existe um subespaço fechado $N$ de $E$, de codimensão finita em $E$, tal que $\left\|\left.T\right|_{N}\right\| \leq \epsilon$ então $T$ é compacto.

Demonstração: Dado $\epsilon>0$, por hipótese, existe $N$ subespaço fechado de codimensão finita de $E$, tal que $\left\|\left.T\right|_{N}\right\| \leq \epsilon$. Logo, pelo teorema 1.1 .17 existem $v_{1}, v_{2}, \ldots, v_{n}$ em $E$ tais que

$$
E=N \oplus\left[v_{1}, \ldots, v_{n}\right]
$$

Segue que existem $x_{1}^{\prime}, x_{2}^{\prime}, \ldots, x_{n}^{\prime}$ em $E^{\prime}$ tais que todo $x$ em $E$ tem uma única representação da forma:

$$
x=u+\sum_{i=1}^{n} x_{i}^{\prime}(x) v_{i}, u \text { em } N
$$

$\operatorname{com} \sum_{i=1}^{n} x_{i}^{\prime}(x) v_{i}$, a projeção de $E$ sobre $\left[v_{1}, \ldots, v_{n}\right]$

Como $\left\|\left.T\right|_{N}\right\| \leq \epsilon$, segue que:

$$
\|T x\| \leq \epsilon\|u\|+\sum_{i=1}^{n}\left|x_{i}^{\prime}(x)\right|\left\|T v_{i}\right\| .
$$


De 1.4,

$$
\|u\| \leq\|x\|+\sum_{i=1}^{n}\left|x_{i}^{\prime}(x)\right|\left\|v_{i}\right\|
$$

De 1.5 e 1.6 e tomando-se $K=\max \left\{\left\|T v_{i}\right\|,\left\|v_{i}\right\|: 1 \leq i \leq n\right\}$, segue que

$$
\|T x\| \leq \epsilon\|x\|+\epsilon K \sum_{i=1}^{n}\left|x_{i}^{\prime}(x)\right|+K \sum_{i=1}^{n}\left|x_{i}^{\prime}(x)\right|,
$$

para cada $x$ em $E$.

Dado $\eta>0$, pelo lema 1.4.10, existem $x_{1}, x_{2}, \ldots, x_{m}$ em $B_{E}$ tais que dado $v$ em $B_{E}$, existe $x_{k}$ tal que

$$
\sum_{i=1}^{n}\left|x_{i}^{\prime} v-x_{i}^{\prime} x_{k}\right| \leq \eta
$$

Substituindo-se $x$ por $v-x_{k}$ em 1.7 e observando-se que $\left\|v-x_{k}\right\| \leq 2$, temos que

$$
\left\|T v-T x_{k}\right\| \leq 2 \epsilon+\epsilon K \eta+K \eta \text {. }
$$

Como $\epsilon>0$ e $\eta>0$ são arbitrários, segue que $T\left(B_{E}\right)$ é totalmente limitado, e logo $\mathrm{T}$ é compacto.

Teorema 1.4.12. Sejam $E$ e $F$ espaços de Banach, e $T \in L(E, F)$. Então $T \in K(E, F)$ se, e somente se, existem $M$ um subespaço fechado de $c_{0}, S \in K(E, M)$ e $R \in K(M, F)$ tais que $T=R \circ S$.

Demonstração: Ver Aliprantis [1], pág. 269, teorema 16.5.

Na próxima seção utilizaremos a seguinte proposição para mostrar que nem sempre $K(E, E)$ é complementado em $L(E, E)$.

Proposição 1.4.13. Seja E um espaço de Banach com base de Schauder. Se $E^{\prime}$ é separável, então $K(E, E)$ é separável.

Demonstração: Ver [9], pág. 205, proposição 7.5 .

Definição 1.4.14. (Operadores completamente contínuos)

Sejam E e F espaços de Banach, e $T: E \rightarrow F$ um operador linear contínuo. Dizemos que $T$ é completamente contínuo se para cada seqüência $\left(x_{n}\right)_{n}$ em E tal que $x_{n} \stackrel{\omega}{\rightarrow} x$ para algum $x$ em E temos que $T x_{n} \rightarrow T x$. 
Proposição 1.4.15. Sejam E e F espaços de Banach, e $T: E \rightarrow F$ um operador linear. Se $T$ é compacto, então $T$ é completamente contínuo.

Demonstração: Seja $\left(x_{n}\right)_{n}$ uma seqüência em $E$ tal que $x_{n} \stackrel{\omega}{\rightarrow} x$ para algum $x$ em $E$. Então, pelo teorema $1.2 .10, T x_{n} \stackrel{\omega}{\rightarrow} T x$. Suponhamos que $T x_{n} \nrightarrow T x$.

Então existe $\left(T x_{n k}\right)_{k}$ tal que $\left\|T x_{n k}-T x\right\| \geq \epsilon$ para algum $\epsilon>0$ e para todo $k \in \mathbb{K}$. Como $\left(x_{n}\right)_{n}$ é fracamente convergente, pela proposição $1.2 .9,\left(x_{n}\right)_{n}$ é limitada. Então, como $T$ é um operador compacto, existe uma subseqüência convergente $T\left(x_{n k l}\right)_{l}$. Suponhamos que $T x_{n k l} \rightarrow y$. Então $T x_{n k l} \stackrel{\omega}{\rightarrow} y$. Logo $y=T x$. Portanto $\left\|T x_{n k l}-T x\right\| \rightarrow$ 0 , uma contradição.

No capítulo 3, utilizamos alguns resultados de operadores compactos em espaços de Hilbert. Apresentamos agora estes resultados, aqui, $H$ e $K$ denotarão espaços de Hilbert $\mathrm{e}<\cdot, \cdot>$ denotará um produto interno.

Definição 1.4.16. Seja $H$ um espaço de Hilbert. Um subconjunto $\left\{h_{j}, j \in J\right\}$ de $H$ é dito ortonormal se $\left\|h_{j}\right\|=1$ para todo $j e<h_{j}, h_{i}>=0$ para todo $i \neq j$.

Definição 1.4.17. Sejam $H$ um espaço de Hilbert $e\left\{h_{j}, j \in J\right\}$ um subconjunto ortonormal em $H$. Se o subespaço gerado pela família $\left\{h_{j}, j \in J\right\}$ é denso em $H$ então $\left\{h_{j}, j \in J\right\}$ é dito completo e cada $x \in H$ é da forma $x=\sum_{j} \alpha_{j} h_{j} \operatorname{com} \sum_{j}\left|\alpha_{j}\right|^{2}<\infty$.

Proposição 1.4.18. Seja $H$ um espaço de Hilbert. Então todo conjunto ortonormal em $H$ pode ser estendido a um subconjunto ortonormal completo.

Demonstração: Ver Pedersen [17], pág. 83, proposição 3.1.12.

Proposição 1.4.19. Seja $H$ um espaço de Hilbert separável de dimensão infinita. Então $H$ é isométrico a $\ell_{2}$.

Demonstração: Ver Retherford [22], pág.25.

Teorema 1.4.20 (Teorema da Representação de Riesz). Sejam $H$ um espaço de Hilbert e $f \in H^{\prime}$. Então existe um único $z \in H$ tal que $f(x)=<x, z>$ para todo $x \in H$. 
Demonstração: Se $f=0$ então o resultado é válido tomando $z=0$. Assim podemos supor que $f \neq 0$. Seja $M=\operatorname{ker} f$. Então $M$ é um subespaço fechado de $H$, e como $f \neq 0$, $M \neq H, \log M^{\perp} \neq\{0\}$. Portanto existe $z_{0} \in M^{\perp}, z_{0} \neq 0$.

Dado $x \in H$, tomemos $z=f(x) z_{0}-f\left(z_{0}\right) x$. Temos que $f(z)=0$, logo $z \in M$. Assim, temos que $0=<z, z_{0}>=<f(x) z_{0}-f\left(z_{0}\right) x, z_{0}>=f(x)<z_{0}, z_{0}>-f\left(z_{0}\right)<x, z_{0}>$. Observando que $<z_{0}, z_{0}>=\left\|z_{0}\right\|^{2} \neq 0$, segue que

$$
f(x)=\frac{f\left(z_{0}\right)}{\left\|z_{0}\right\|^{2}}<x, z_{0}>.
$$

Tomando $z=\frac{\overline{f\left(z_{0}\right)}}{\left\|z_{0}\right\|^{2}} z_{0}$, temos que $f(x)=<x, z>$.

Vamos agora mostrar que $z$ é único. Suponhamos então que existe $z^{\prime} \in H$ tal que $f(x)=<x, z>=<x, z^{\prime}>$ para todo $x \in H$. Então $<x, z-z^{\prime}>=0$ para todo $x \in H$. Em particular, para $x=z-z^{\prime}$, temos que $<z-z^{\prime}, z-z^{\prime}>=\left\|z-z^{\prime}\right\|^{2}=0$. Logo $z=z^{\prime}$. Portanto $z$ é único.

Se $H$ é um espaço de Hilbert, denotaremos por $l_{2}^{\omega}(H)$ o espaço de todas as seqüências $\left(x_{n}\right)_{n}$ de $H$ tais que para todo $f \in H^{\prime}$ temos que $\sum_{n=1}^{\infty}\left|f\left(x_{n}\right)\right|^{2}<\infty$.

Lema 1.4.21. Sejam $H$ um espaço de Hilbert e $\left(h_{n}\right)_{n}$ uma seqüência ortonormal em $H$. Então $\left(h_{n}\right)_{n} \in l_{2}^{\omega}(H)$.

Demonstração: Seja $\left(h_{n}\right)_{n}$ uma seqüência ortonormal em $H$. Vamos mostrar que $\sum_{n}\left|f\left(h_{n}\right)\right|^{2}<\infty$ para cada $f \in H^{\prime}$.

Pelo teorema da representação de Riesz (teorema 1.4.20), temos que para cada $f \in H^{\prime}$, existe um único $y \in H$ tal que $f\left(h_{n}\right)=<h_{n}, y>$, para todo $n \in \mathbb{I N}$. Assim $\sum_{n}\left|f\left(h_{n}\right)\right|^{2}=$ $\sum_{n}\left|<h_{n}, y>\right|^{2}$.

Pela proposição 1.4.18 $\left(h_{n}\right)_{n}$ pode ser estendida uma uma seqüência ortonormal completa $\left(h_{\alpha}\right)_{\alpha} \operatorname{com} \sum_{\alpha}\left|<h_{\alpha}, y>\right|^{2}<\infty$. Como $\sum_{n}\left|<h_{n}, y>\right|^{2}<\sum_{\alpha}\left|<h_{\alpha}, y>\right|^{2}$, segue que $\sum_{n}\left|f\left(h_{n}\right)\right|^{2}<\infty$, para cada $f \in H^{\prime}$. Portanto $\left(h_{n}\right)_{n} \in l_{2}^{\omega}(H)$, e conseqüentemente $\left(h_{n}\right)_{n}$ converge fracamente a zero.

Se $H$ e $K$ são espaços de Hilbert e $T: H \rightarrow K$ é um operador linear contínuo tal que $\lim _{n}\left\|T h_{n}\right\|=0$ para toda seqüência ortonormal $\left(h_{n}\right)_{n}$ em $H$, então $T$ é compacto. 
Este resultado será utilizado no capítulo 3. No que segue apresentamos este resultado precedido de um lema que será utilizado em sua demonstração.

Se $T: H \rightarrow K$ é um operador linear contínuo entre espaços de Hilbert, denotaremos por $T^{\times}: K \rightarrow H$ o operador adjunto de Hilbert de $T$ tal que $\langle T x, y\rangle=\left\langle x, T^{\times} y\right\rangle$ para todo $x \in H$ e $y \in K$. Um operador linear contínuo $T: H \rightarrow H$, com $H$ um espaço de Hilbert, é dito auto-adjunto se $T^{\times}=T$.

Lema 1.4.22. Sejam $H$ um espaço de Hilbert e $Q \in L(H, H)$ uma projeção ortogonal sobre um subespaço fechado $M \subset H$. Então $Q$ é um operador auto-adjunto.

Demonstração: Sendo $M$ um subespaço fechado de $H$, temos que $H=M \oplus M^{\perp}$, com $M^{\perp}=\{n \in H:\langle n, m>=0$, para todo $m$ em $M\}$. Como $Q$ é uma projeção de $H$ sobre $M$, temos que $\operatorname{ker} Q=M^{\perp}$.

Para todos $x, y \in H, x=m+n$ e $y=m_{1}+n_{1}$, com $m, m_{1} \in M$ e $n, n_{1} \in M^{\perp}$, podemos escrever:

$$
\begin{gathered}
<Q x, y>=<m, m_{1}+n_{1}>=<m, m_{1}>= \\
=<m, m_{1}>+<n, m_{1}>=<m+n, m_{1}>=<x, Q y>=<Q^{\times}(x), y>.
\end{gathered}
$$

Logo para todos $x, y \in H$ temos que $\left\langle\left(Q-Q^{\times}\right)(x), y>=0\right.$. Tomando-se $y=$ $\left(Q-Q^{\times}\right)(x)$ segue que para todo $x \in H,\left\langle\left(Q-Q^{\times}\right)(x),\left(Q-Q^{\times}\right)(x)>=0 \mathrm{e}\right.$ conseqüentemente $Q^{\times}=Q$.

Proposição 1.4.23. Sejam $H$ e $K$ espaços de Hilbert, e $T: H \rightarrow K$ um operador linear contínuo. Então $T$ é compacto se, e somente se, $\lim _{n}\left\|T h_{n}\right\|=0$ para toda seqüência ortonormal $\left(h_{n}\right)_{n}$ em $H$.

Demonstração: Seja $\left(h_{n}\right)_{n}$ uma seqüência ortonormal em $H$. Pelo lema 1.4.21, $\left(h_{n}\right)_{n}$ converge fracamente a zero. Se $T$ é compacto então, pela proposição $1.4 .15, T$ é completamente contínuo. Segue que $\lim _{n}\left\|T h_{n}\right\|=0$.

Para a recíproca, mostraremos que se $T: H \rightarrow K$ é um operador linear não compacto então $\lim _{n}\left\|T h_{n}\right\|>0$ para alguma seqüência ortonormal $\left(h_{n}\right)_{n}$ em $H$.

Vamos definir uma seqüência $\left(h_{n}\right)_{n}$ indutivamente. 
Sendo $T$ um operador linear não compacto, $T$ não é limite de qualquer seqüência de operadores de posto finito. Assim, existe $\eta>0$ tal que $\|T-P\|>\eta$ para cada operador $P$ com posto finito. Em particular, $\|T\|>\eta$ (basta tomar $P$ como o operador nulo). Pela definição de norma, existe $h_{1} \in H,\left\|h_{1}\right\|=1$ tal que $\left\|T h_{1}\right\|>\eta$.

Suponhamos que já estejam definidos os elementos $h_{1}, \ldots, h_{m} \in H,\left\|h_{i}\right\|=1$, com $\left\|T h_{i}\right\|>\eta \mathrm{e}<h_{i}, h_{j}>=\delta_{i, j}$ para $i, j=1, \ldots, m$, para algum $m \geq 1$. Denotemos por $Q$ a projeção ortogonal de $H$ sobre o espaço $E_{m}=\left[h_{1}, \ldots, h_{m}\right]$. Consideremos $P=T \circ Q$. Então, $P$ é linear e de posto finito, donde segue que $\|T-P\|>\eta$. Escolhamos assim $h \in H$ tal que $\|h\|=1$ e $\|(T-P) h\|>\eta>0$. Temos $T h \neq P h$, donde $Q h-h \neq 0$. Consideremos $h_{m+1}=\|h-Q h\|^{-1}(h-Q h)$. Temos que $\left\|h_{m+1}\right\|=1$ e ainda temos que $T h_{m+1}=\|h-Q h\|^{-1}(T h-T \circ Q h)=\|h-Q h\|^{-1}(T-P) h$.

Como $\|h\|=1$ e $Q$ é uma projeção ortogonal, segue que $\|h-Q h\| \leq\|h\|=1$, logo $\left\|T h_{m+1}\right\|=\|h-Q h\|^{-1}\|(T-P) h\|>1 . \eta>\eta$. Portanto, como $Q h_{m+1}=0$, segue que $h_{m+1}$ é ortogonal a todo elemento da imagem de $Q$.

De fato, se $y \in \operatorname{Im} Q, y=Q x, x \in H$ e utilizando o lema 1.4 .22 (e observando que $\operatorname{dim}(\operatorname{Im} Q)<\infty)$, temos que:

$$
<y, h_{m+1}>=<Q x, h_{m+1}>=<x, Q^{\times} h_{m+1}>=<x, Q h_{m+1}>=0 .
$$

Em particular, temos $\left\langle h_{m+1}, h_{i}\right\rangle=0, i=\ldots, m$. Fica então definida a seqüência ortonormal $\left(h_{i}\right)_{i}$ em $H$ tal que $\lim _{n}\left\|T h_{n}\right\|>\eta>0$.

Apresentamos a seguir a definição de Operadores fracamente compactos e somente alguns resultados sobre estes que utilizaremos posteriormente em demonstrações de resultados no capítulo 3 .

Definição 1.4.24. (Operadores fracamente compactos) Um operador linear $T: E \rightarrow F$ entre espaços de Banach é fracamente compacto se para todo subconjunto B limitado de $E$, temos que $T(B)$ é um subconjunto relativamente fracamente compacto de $F$.

$O$ conjunto dos operadores fracamente compactos de $E$ em $F$ será denotado por $K^{\omega}(E, F)$. Não é difícil mostrar que $K^{\omega}(E, F)$ é um subespaço de $L(E, F)$.

Segue do teorema de Eberlien-Smulian (teorema 1.2.16), a seguinte proposição. 
Proposição 1.4.25. Sejam $E$ e $F$ espaços de Banach, e $T: E \rightarrow F$ um operador linear. Então são equivalentes.

(a) $T$ é fracamente compacto.

(b) Toda seqüência limitada $\left(x_{n}\right)_{n}$ em E admite uma subseqüência $\left(x_{n j}\right)_{j}$ tal que $\left(T x_{n j}\right)_{j}$ converge fracamente.

Teorema 1.4.26 (Gantmacher). Um operador linear contínuo entre espaços de Banach é fracamente compacto se, e somente se, seu adjunto é fracamente compacto.

Demonstração: Ver Megginson [16], pág. 343, teorema 3.5.13.

Teorema 1.4.27. Sejam $\Omega$ um espaço de Hausdorff compacto e $E$ um espaço de Banach qualquer tal que $c_{0} \nLeftarrow$ E. Então todo operador linear contínuo de $C(\Omega)$ em $E$ é fracamente compacto.

Demonstração: Ver Pelczynski [18], pág. 219, teorema 5.

\section{$1.5 \mathrm{O}$ espaço $K\left(\ell_{2}, \ell_{2}\right)$}

Ao longo dessa dissertação estudamos algumas caracterizações de operadores compactos entre espaços de Banach. Como trabalhamos com espaços que contém cópias isométricas de $c_{0}$ e com a noção de subespaços complementados, decidimos por incluir nesta seção exemplo de um espaço formado por operadores compactos que possui cópia de $c_{0}$ e não é complementado em $L\left(\ell_{2}, \ell_{2}\right)$.

Proposição 1.5.1. O espaço $c_{0}$ é isométrico a um subespaço de $K\left(\ell_{2}, \ell_{2}\right)$.

Demonstração: Seja $\varphi$ uma aplicação linear de $c_{0} \operatorname{em} K\left(\ell_{2}, \ell_{2}\right)$ dada por $\varphi(a)=T(a)$ para cada $a=\left(a_{i}\right)_{i} \in c_{0}$, com $T(a): \ell_{2} \rightarrow \ell_{2}$ dado por $T(a)(x)=\left(a_{i} x_{i}\right)_{i}$ para cada $x=\left(x_{i}\right)_{i} \in \ell_{2}$.

De

$$
\left(\sum\left|a_{i} x_{i}\right|^{2}\right)^{1 / 2}=\left(\sum\left|a_{i}\right|^{2}\left|x_{i}\right|^{2}\right)^{1 / 2} \leq\|a\|_{\infty}\left(\sum\left|x_{i}\right|^{2}\right)^{1 / 2}=\|a\|_{\infty}\|x\|_{2},
$$

temos que o operador $T(a)$ está bem definido e é contínuo com $\|T(a)\| \leq\|a\|_{\infty}$. 
Dado $\epsilon>0$, existe $n_{0} \in \mathbb{N}$ tal que $\left|a_{n_{0}}\right|>\|a\|_{\infty}-\epsilon$. Como $T(a)\left(e_{n_{0}}\right)=a_{n_{0}} e_{n_{0}} \mathrm{e}$ $\left\|e_{n_{0}}\right\|=1$, segue que

$$
\left\|T(a)\left(e_{n_{0}}\right)\right\|_{2}=\left|a_{n_{0}}\right|>\|a\|_{\infty}-\epsilon .
$$

Fazendo $\epsilon \rightarrow 0$, temos que $\|T(a)\| \geq\|a\|_{\infty}$.

Logo $\|T(a)\|=\|a\|_{\infty}$ e $\varphi$ é uma isometria.

Vamos agora mostrar que $T(a)$ é compacto. Para cada $n \in \mathbb{N}$, seja $T_{n}(a): \ell_{2} \rightarrow \ell_{2}$ um operador linear dado por $T_{n}(a)(x)=\left(a_{1} x_{1}, a_{2} x_{2}, \ldots, a_{n} x_{n}, 0,0, \ldots\right)$ para cada $x=$ $\left(x_{1}, x_{2}, \ldots\right) \in \ell_{2}$.

Para cada $n \in \mathbb{N}$ e $x=\left(x_{i}\right)_{i} \in \ell_{2}$,

$$
\left(\sum_{i=1}^{n}\left|a_{i} x_{i}\right|^{2}\right)^{1 / 2}=\left(\sum_{i=1}^{n}\left|a_{i}\right|^{2}\left|x_{i}\right|^{2}\right)^{1 / 2} \leq\|a\|_{\infty}\left(\sum_{i=1}^{n}\left|x_{i}\right|^{2}\right)^{1 / 2} \leq\|a\|_{\infty}\|x\|_{2},
$$

logo temos que o operador $T_{n}(a)$ está bem definido e é contínuo.

Para cada $n \in \mathbb{N}, T_{n}(a)$ é compacto pois é um operador de posto finito. Vamos agora provar que $T(a)$ é compacto.

Dado $\epsilon>0$, existe $n_{1} \in \mathbb{N}$ tal que $n \geq n_{1} \Rightarrow\left|a_{n}\right|<\epsilon$.

Para $n \geq n_{1}$,

$$
\begin{gathered}
\left\|\left(T(a)-T_{n}(a)\right)(x)\right\|_{2}=\left\|T(a)(x)-T_{n}(a)(x)\right\|_{2}= \\
=\left(\sum_{i=n+1}^{\infty}\left|a_{i} x_{i}\right|^{2}\right)^{1 / 2} \leq \sup _{i>n}\left|a_{i}\right|\left(\sum\left|x_{i}\right|^{2}\right)^{1 / 2} \leq \\
\leq \sup _{i>n}\left|a_{i}\right|\|x\|_{2}
\end{gathered}
$$

Logo $\left\|T(a)-T_{n}(a)\right\| \leq \sup _{i>n}\left|a_{i}\right|<\epsilon$. Portanto $T(a)$ é compacto.

Corolário 1.5.2. O espaço $K\left(\ell_{2}, \ell_{2}\right)$ não é reflexivo.

Demonstração: Pela proposição 1.5.1, $c_{0}$ é isométrico a um subespaço de $K\left(\ell_{2}, \ell_{2}\right)$. Como $c_{0}$ não é reflexivo, segue o resultado.

Proposição 1.5.3. O espaço $K\left(\ell_{2}, \ell_{2}\right)$ não é complementado em $L\left(\ell_{2}, \ell_{2}\right)$. 
Demonstração: Vamos supor por absurdo que $K\left(\ell_{2}, \ell_{2}\right)$ seja complementado em $L\left(\ell_{2}, \ell_{2}\right)$. Ou seja, existe uma projeção $P: L\left(\ell_{2}, \ell_{2}\right) \rightarrow K\left(\ell_{2}, \ell_{2}\right)$. Vamos mostrar que com tal hipótese podemos exibir uma projeção $Q$ em $\ell_{\infty} \operatorname{com} \operatorname{Im} Q=c_{0}$, chegando assim num absurdo pelo teorema 1.1.32.

Segue da proposição 1.5.1, que existe um subespaço fechado $M$ de $K\left(\ell_{2}, \ell_{2}\right)$ isométrico a $c_{0}$. Seja $\varphi: c_{0} \rightarrow M$ tal isometria dada por $\varphi(a)=T(a)$ para cada $a=\left(a_{i}\right)_{i} \in c_{0}$, com $T(a): \ell_{2} \rightarrow \ell_{2}$ dado por $T(a)(x)=\left(a_{i} x_{i}\right)_{i}$ para cada $x=\left(x_{i}\right)_{i} \in \ell_{2}$.

Podemos estender $\varphi$ para $\tilde{\varphi}: \ell_{\infty} \rightarrow L\left(\ell_{2}, \ell_{2}\right)$ a aplicação linear contínua dada por $\tilde{\varphi}(a)(x)=T(a)(x)=\left(a_{i} x_{i}\right)_{i}$ para cada $a=\left(a_{i}\right)_{i} \in \ell_{\infty}$ e cada $x=\left(x_{i}\right)_{i} \in \ell_{2}$. Como vimos na proposição 1.1.30, $\tilde{\varphi}$ é uma isometria de $\ell_{\infty}$ sobre um subespaço $N$ de $L\left(\ell_{2}, \ell_{2}\right)$.

Agora, como $\ell_{2}$ é separável, segue da proposição 1.4 .13 que $K\left(\ell_{2}, \ell_{2}\right)$ é separável. Então pelo teorema 1.1.31, $M$ é complementado em $K\left(\ell_{2}, \ell_{2}\right)$. Logo, obtemos uma projeção linear contínua $R: K\left(\ell_{2}, \ell_{2}\right) \rightarrow M$.

Assim definimos $Q: \ell_{\infty} \rightarrow c_{0}$ como o operador linear contínuo dado por $Q=\varphi^{-1} \circ$ $R \circ P \circ \tilde{\varphi}$. Afirmamos que $Q$ é sobrejetor e $Q^{2}=Q$. De fato, para cada $a \in c_{0}$, existe $T \in M$ tal que $\left(\varphi^{-1}\right)(T)=a$. Usando que $R$ é uma projeção de $K\left(\ell_{2}, \ell_{2}\right)$ sobre $M$, segue que $R(T)=T$ e como $P$ também é uma projeção de $L\left(\ell_{2}, \ell_{2}\right)$ sobre $K\left(\ell_{2}, \ell_{2}\right)$, segue que $P(T)=T$. Agora $\tilde{\varphi}(a)=\varphi(a)=T$. Assim $Q(a)=\left(\varphi^{-1} \circ R \circ P \circ \tilde{\varphi}\right)(a)=\varphi^{-1} \circ R \circ P(T)=$ $\varphi^{-1}(T)=a$.

Vamos agora provar que $Q^{2}=Q$. Para cada $a \in \ell_{\infty}$, temos que $\left(\varphi^{-1} \circ R \circ P \circ \tilde{\varphi}\right)(a) \in c_{0}$, $\log 0 \tilde{\varphi}\left(\varphi^{-1}(R(P(\tilde{\varphi}(a))))\right)=\varphi \circ \varphi^{-1} \circ R \circ P(\tilde{\varphi}(a))=R(P(\tilde{\varphi}(a)))$.

Usando que $R(P(\tilde{\varphi}(a))) \in M \subset K\left(\ell_{2}, \ell_{2}\right)$, segue que $P(R(P(\tilde{\varphi}(a))))=R(P(\tilde{\varphi}(a)))$. $\operatorname{Assim} Q^{2}(a)=\left(\varphi^{-1} \circ R \circ P \circ \tilde{\varphi} \circ \varphi^{-1} \circ R \circ P \circ \tilde{\varphi}\right)(a)=\left(\varphi^{-1}(R(R(P(\tilde{\varphi}(a)))))\right)=$ $\left(\varphi^{-1}(R(P(\tilde{\varphi}(a))))\right)=Q(a)$, concluindo assim que $Q$ é uma projeção de $\ell_{\infty}$ sobre $c_{0}$, um absurdo. 
O espaço $K\left(\ell_{2}, \ell_{2}\right)$ 


\section{Capítulo 2}

\section{Uma caracterização de operadores compactos atráves de $\ell_{1}$}

O objetivo desse capítulo é apresentar algumas caracterizações dos espaços de Banach $F$ para os quais todos os operadores compactos $T: E \rightarrow F$ de um espaço de Banach $E$ em $F$ admitam uma particular representação em séries.

Os resultados que apresentamos foram estudados no texto científico de Randtke [21]. Entre eles o teorema 2.10 prova que, a menos de isomorfismo, $\ell_{1}$ é o único espaço de Banach $E$ tal que todo operador linear compacto $T: F \rightarrow E$ tem uma representação da forma $T x=\sum g_{n}(x) u_{n}$, para cada $x \in F$, com $F$ um espaço de Banach, $\left(u_{n}\right)_{n}$ base normalizada de $E$ e $\sum g_{n}$ uma série $\omega^{*}$ incondicionalmente convergente no dual topológico $F^{\prime}$ de $F$.

Convém ressaltar que alguns dos resultados apresentados no trabalho de Randtke [21] são conseqüências da teoria de Grothendieck para produtos tensoriais (ver Grothendieck [11]). No entanto, as demonstrações apresentadas em [21] são muito mais simples e independem da teoria de produtos tensoriais.

Começamos apresentando dois resultados de Randtke [20] que utilizaremos na demonstração da proposição 2.4. Em [20] estes resultados são enunciados para espaços normados mas, de acordo com o contexto desta dissertação, os enunciamos para espaços de Banach.

Neste capítulo, a menos de menção explícita do contrário, denotaremos por $\left(e_{n}\right)_{n}$ a 
Uma caracterização de operadores compactos através de $\ell_{1}$

base canônica de $l_{1}$.

Teorema 2.1. Sejam E e F espaços de Banach, e $T \in L(E, F)$. Se $T$ é compacto então existe uma seqüência $\left(x_{n}^{\prime}\right)_{n} \subset E^{\prime}$ tal que $\left\|x_{n}^{\prime}\right\| \rightarrow 0 e\|T(x)\| \leq \sup _{n}\left|x_{n}^{\prime}(x)\right|$ para todo $x \in E$.

Demonstração: Se $T$ é compacto, então pelo teorema de Schauder (teorema 1.4.9), $T^{*}: F^{\prime} \rightarrow E^{\prime}$ também é compacto. Então $\overline{T^{*}\left(B_{F^{\prime}}\right)}$ é compacto em $E^{\prime}$, logo pelo teorema 1.1.33, existe uma seqüência $\left(x_{n}^{\prime}\right)_{n} \subset E^{\prime}$ tal que $\lim _{n}\left\|x_{n}^{\prime}\right\|=0$ e $\overline{T^{*}\left(B_{F^{\prime}}\right)} \subset$ $\overline{c o}\left(\left(x_{n}^{\prime}\right)_{n}\right)$.

Assim, se $T^{*}\left(y^{\prime}\right) \in \overline{T^{*}\left(B_{F^{\prime}}\right)}$, existe $\left(w_{m}\right)_{m} \in \operatorname{co}\left(\left(x_{n}^{\prime}\right)_{n}\right)$ tal que $w_{m} \rightarrow T^{*}\left(y^{\prime}\right)$. Agora, dado $\epsilon>0$, existe $n_{0} \in \mathbb{I N}$ tal que $\left\|T^{*}\left(y^{\prime}\right)-w_{n_{0}}\right\|<\epsilon$, isto é, $\left\|T^{*}\left(y^{\prime}\right)\right\|<\epsilon+\left\|w_{n_{0}}\right\|$.

Como $w_{n_{0}}=\sum_{i=1}^{l} \lambda_{w_{n_{0}, i}} x_{i}^{\prime}$, com $\sum_{i=1}^{l} \lambda_{w_{n_{0}, i}}=1$ e $\lambda_{w_{n_{0}}} \geq 0$, segue que

$$
\left\|T^{*}\left(y^{\prime}\right)\right\|<\epsilon+\left\|w_{n_{0}}\right\|=\epsilon+\sum_{i=1}^{l} \lambda_{w_{n_{0}, i}} x_{i}^{\prime} .
$$

Conseqüentemente, para cada $x \in E$,

$\left|\left(T^{*}\left(y^{\prime}\right)\right)(x)\right|<\epsilon+\left|\sum_{i=1}^{l} \lambda_{w_{n_{0}, i}} x_{i}^{\prime}(x)\right| \leq \epsilon+\sum_{i=1}^{l} \lambda_{w_{n_{0}, i}}\left|x_{i}^{\prime}(x)\right| \leq \epsilon+\sup _{k}\left|x_{k}^{\prime}(x)\right|$.

Logo, $\left|\left(T^{*}\left(y^{\prime}\right)\right)(x)\right|<\epsilon+\sup _{i}\left|x_{i}^{\prime}(x)\right|$.

Então para cada $x \in E$, temos que $\|T(x)\|=\sup \left\{y^{\prime}(T(x)): y^{\prime} \in B_{F^{\prime}}\right\}=$ $\sup \left\{\left(T^{*}\left(y^{\prime}\right)\right)(x): y^{\prime} \in B_{F^{\prime}}\right\} \leq \sup _{n}\left|x_{n}^{\prime}(x)\right|+\epsilon$. Como a desigualdade vale para todo $\epsilon>0$, segue o resultado.

Lema 2.2. Sejam $F$ um espaço de Banach e $T: c_{0} \rightarrow F$ um operador linear. Se $T$ é compacto, então existe uma seqüência $\lambda=\left(\lambda_{n}\right)_{n}$ em co tal que, para cada $\mu=\left(\mu_{n}\right)_{n}$ em $c_{0}$,

$$
\|T \mu\| \leq \sup _{n}\left\{\left|\lambda_{n}\right|^{2}\left|\mu_{n}\right|\right\} .
$$

Demonstração: Se $T$ é compacto, então pelo teorema 2.1 existe uma seqüência $\left(x_{n}^{\prime}\right)_{n} \in c_{o}^{\prime}$ tal que $\left\|x_{n}^{\prime}\right\| \rightarrow 0$ e $\|T(\mu)\| \leq \sup _{n}\left|x_{n}^{\prime}(\mu)\right|$ para todo $\mu=\left(\mu_{n}\right)_{n} \in c_{0}$.

Para concluir a demonstração do lema, vamos definir operadores auxiliares da seguinte maneira: considere os operadores lineares 


$$
\begin{gathered}
c_{0} \stackrel{S}{\longrightarrow} S\left(c_{0}\right) \stackrel{R}{\longrightarrow} c_{0} \\
x=\left(x_{m}\right)_{m} \rightarrow\left(\left\|x_{m}^{\prime}\right\| x_{m}\right)_{m} \rightarrow\left(x_{m}^{\prime}(x)\right)_{m} .
\end{gathered}
$$

Afirmamos que $S$ e $R \circ S$ são contínuos. De fato,

$$
\|S(x)\|=\left\|\left(\left\|x_{m}^{\prime}\right\| x_{m}\right)_{m}\right\|=\sup _{m}\left\|x_{m}^{\prime}\right\|\left|x_{m}\right| \leq C \sup _{m}\left|x_{m}\right|=C\|x\|
$$

e

$$
\left\|R\left(\left(\left\|x_{m}^{\prime}\right\| x_{m}\right)_{m}\right)\right\|=\left\|\left(x_{m}^{\prime}(x)\right)_{m}\right\|=\sup _{m}\left|x_{m}^{\prime}(x)\right| \leq \sup _{m}\left\|x_{m}^{\prime}\right\|\|x\| \leq C\|x\| .
$$

Assim $\left.\left\|R\left(\left(\left\|x_{m}^{\prime}\right\| x_{m}\right)_{m}\right)\right\|=\left\|\left(x_{m}^{\prime}(x)\right)_{m}\right\| \leq\|R\| \|\left(\left\|x_{m}^{\prime}\right\| x_{m}\right)_{m}\right) \|$. Chamando $\left|\lambda_{m}\right|^{2}=\|R\|\left\|x_{m}^{\prime}\right\|$, temos que $\lambda_{n} \rightarrow 0$ e $\sup _{m}\left|x_{m}^{\prime}(x)\right| \leq\|R\| \sup _{m}\left\|x_{m}^{\prime}\right\|\left|x_{m}\right|=$ $\sup _{m}\left|\lambda_{m}\right|^{2}\left|x_{m}\right|$.

Conseqüentemente, $\|T(\mu)\| \leq \sup _{m}\left|\lambda_{m}\right|^{2}\left|\mu_{m}\right|$, para todo $\mu=\left(\mu_{n}\right)_{n} \in c_{0}$.

Proposição 2.3. Sejam $E$ um espaço de Banach e $T: c_{0} \rightarrow E$ um operador linear. Se $T$ é compacto, entâo existem uma seqüência $\lambda=\left(\lambda_{n}\right)_{n}$ em $c_{0}$ e uma série incondicionalmente convergente $\sum y_{n}$ em $E$, tais que, para cada $\mu=\left(\mu_{n}\right)_{n}$ em $c_{0}, T \mu=\sum \lambda_{n} \mu_{n} y_{n}$.

Demonstração: Se $T: c_{0} \rightarrow E$ é compacto, então pelo lema 2.2, existe uma seqüência $\lambda=\left(\lambda_{n}\right)_{n}$ em $c_{0}$ tal que para cada $\mu=\left(\mu_{n}\right)_{n}$ em $c_{0}$

$$
\|T \mu\| \leq \sup _{n}\left\{\left|\lambda_{n}\right|^{2}\left|\mu_{n}\right|\right\}
$$

Se $\lambda_{n}=0$ para todo $n \in \mathbb{N}$, então o resultado é imediato; caso contrário, efetuando convenientes trocas de posição na seqüência, podemos assumir que $\lambda_{n} \neq 0$ para naturais convenientes.

Para cada $n$, seja $y_{n}=T\left[\left(\lambda_{n}\right)^{-1} e_{n}\right]$, com $\left(e_{n}\right)_{n}$ base canônica de $c_{0}$. Vamos mostrar que a série $\sum y_{n}$ é incondicionalmente convergente. Para cada $\xi=\left(\xi_{n}\right)_{n} \neq 0$ em $\ell_{\infty}$, como $\lambda \in c_{0}$, dado $\epsilon>0$, existe $n_{0}$ tal que $\sup \left\{\left|\lambda_{k}\right|: k \geq n_{0}\right\}<\epsilon /\|\xi\|_{\infty}$.

Assim, de 2.1, temos que

$$
\left\|\sum_{k=n_{0}}^{m} \xi_{k} y_{k}\right\|=\left\|T\left[\sum_{k=n_{0}}^{m} \xi_{k}\left(\lambda_{k}\right)^{-1} e_{k}\right]\right\| \leq \sup _{k}\left\{\left|\lambda_{k}\right|^{2}\left|\xi_{k} \| \lambda_{k}\right|^{-1}: k \geq n_{0}\right\}=
$$




$$
=\sup \left\{\left|\lambda_{k} \| \xi_{k}\right|: k \geq n_{0}\right\} \leq\|\xi\|_{\infty} \sup \left\{\left|\lambda_{k}\right|: k \geq n_{0}\right\} \leq \epsilon
$$

Segue que a série $\sum \xi_{n} y_{n}$ é Cauchy somável em $E$ e portanto, pelas proposições 1.3.2 e 1.3.5, temos que a série $\sum y_{n}$ é incondicionalmente convergente em $E$.

Vamos agora mostrar que para cada $\mu=\left(\mu_{n}\right)_{n}$ em $c_{0}, T \mu=\sum \lambda_{n} \mu_{n} y_{n}$.

Como para cada $\mu=\left(\mu_{n}\right)_{n}$ em $c_{0}$, existe $n_{0} \in \mathbb{N}$ tal que $\sum_{k>n_{0}}\left|\mu_{n}\right|<\epsilon /\|T\|$ temos que

$$
\begin{gathered}
\left\|T \mu-\sum_{k=1}^{n_{0}} \lambda_{k} \mu_{k} y_{k}\right\|=\left\|T \mu-\sum_{k=1}^{n_{0}} \lambda_{k} \mu_{k} T\left[\left(\lambda_{k}\right)^{-1} e_{k}\right]\right\|= \\
=\left\|T \mu-\sum_{k=1}^{n_{0}} T\left(\mu_{k} e_{k}\right)\right\|=\left\|T\left[\sum_{k>n_{0}} \mu_{k} e_{k}\right]\right\| \leq\|T\|\left\|\sum_{k>n_{0}} \mu_{k} e_{k}\right\| \leq \epsilon
\end{gathered}
$$

Segue que $T \mu=\sum \lambda_{n} \mu_{n} y_{n}$.

No que segue vamos apresentar alguns resultados cujas demonstrações encontram-se em [21]. A próxima proposição mostra que se $T$ é um operador linear de um espaço de Banach $E$ em $\ell_{1}$, então $T$ é compacto se, e somente se, $T$ tem uma particular representação em séries.

Proposição 2.4. Sejam E um espaço de Banach e $T: E \rightarrow \ell_{1}$ um operador linear. Então são equivalentes:

(1) $T$ é compacto.

(2) Existem uma seqüência $\lambda=\left(\lambda_{n}\right)_{n}$ em $c_{0}$ e uma série incondicionalmente convergente $\sum g_{n}$ em $E^{\prime}$ tais que para cada $x$ em $E$

$$
T x=\sum \lambda_{n} g_{n}(x) e_{n},
$$

$\operatorname{com}\left(e_{n}\right)_{n}$ é a base canônica de $\ell_{1}$.

(3) Existem uma seqüência $\lambda=\left(\lambda_{n}\right)_{n}$ em $c_{0}$ e uma série $\omega^{*}$ incondicionalmente convergente $\sum g_{n}$ em $E^{\prime}$ tais que para cada $x$ em $E$

$$
\|T x\| \leq \sum\left|\lambda_{n}\right|\left|g_{n}(x)\right| .
$$


Demonstração: (1) implica (2). Se $T$ é compacto, então pelo teorema de Schauder (teorema 1.4.9), $T^{*}: \ell_{\infty} \rightarrow E^{\prime}$ também é compacto.

Consideremos $C: c_{0} \rightarrow l_{\infty}$ a inclusão natural, que a cada $\mu \in c_{0}$ associa $C(\mu)=C_{\mu}$, dada por $C_{\mu}(\lambda)=\lambda(\mu)$ para cada $\lambda \in l_{1}$.

Então a aplicação $T^{*} \circ C: c_{0} \rightarrow E^{\prime}$ é compacta e pela proposição 2.3 existem uma sequência $\lambda=\left(\lambda_{n}\right)_{n}$ em $c_{0}$ e uma série incondicionalmente convergente $\sum g_{n}$ em $E^{\prime}$ tal que para cada $\mu=\left(\mu_{n}\right)_{n}$ em $c_{0}$

$$
\left(T^{*} \circ C\right)(\mu)=\sum_{n} \lambda_{n} \mu_{n} g_{n} .
$$

A aplicação $T^{*} \circ C$ está bem definida pois a série $\sum g_{n}$ é incondicionalmente convergente em $E^{\prime}$. Agora para cada $x$ em $E$ e para cada $\mu=\left(\mu_{n}\right)_{n}$ em $c_{0}$, observando-se que $C_{\mu}\left(e_{n}\right)=\mu_{n}$ para cada $n \in \mathbb{N}$, temos que

$$
\left(\left(T^{*} \circ C\right)(\mu)\right)(x)=\sum_{n} \lambda_{n} \mu_{n} g_{n}(x)=C_{\mu}\left(\sum_{n} \lambda_{n} g_{n}(x) e_{n}\right)=\left(\sum_{n} \lambda_{n} g_{n}(x) e_{n}\right)(\mu) .
$$

Por outro lado,

$$
\left(\left(T^{*} \circ C\right)(\mu)\right)(x)=\left(T^{*}\left(C_{\mu}\right)\right)(x)=C_{\mu}(T x)=(T x)(\mu) .
$$

Logo,para cada $x$ em $E$ e para cada $\mu=\left(\mu_{n}\right)_{n}$ em $c_{0}$ :

$$
(T x)(\mu)=\left(\sum_{n} \lambda_{n} g_{n}(x) e_{n}\right)(\mu) .
$$

E assim, como as funções coincidem em todos os pontos do domínio, segue que:

$$
T x=\sum_{n} \lambda_{n} g_{n}(x) e_{n}
$$

(2) implica (3). Por (2), existe uma seqüência $\lambda=\left(\lambda_{n}\right)_{n}$ em $c_{0}$ e uma série incondicionalmente convergente $\sum g_{n}$ em $E^{\prime}$ tal que para cada $x$ em $E$

$$
T x=\sum_{n} \lambda_{n} g_{n}(x) e_{n},
$$

com $\left(e_{n}\right)$ a base canônica de $\ell_{1}$. Agora, pela proposição 1.3 .8 , temos que $\sum g_{n}$ é $\omega^{*}$ incondicionalmente convergente em $E^{\prime}$, e de 2.2 temos que,

$$
\|T x\| \leq \sum_{n}\left|\lambda_{n}\right|\left|g_{n}(x)\right| .
$$


(3) implica (1). Para cada $x$ em $E$ definamos as seguintes funções de $E$ em $\mathbb{R}^{+}$:

$$
\begin{aligned}
& p(x)=\sum_{n=1}^{\infty}\left|g_{n}(x)\right|^{1} ; \\
& p_{k}(x)=\sum_{n=1}^{k}\left|g_{n}(x)\right| .
\end{aligned}
$$

Como $\sum g_{n}$ é $\omega^{*}$ incondicionalmente convergente, $p$ está bem definida. Primeiro vamos mostrar que existe um $M>0$ tal que $p(x)<M\|x\|$ para todo $x \in E$. Para cada $k$, $p_{k}$ é soma finita de funções contínuas, $\operatorname{logo} p_{k}$ é contínua. Assim para cada $k$ e para cada $r \in \mathbb{R}^{+}$, o conjunto $\left\{x \in E: p_{k}(x) \leq r\right\}$ é imagem inversa de um conjunto fechado de $\mathbb{R}^{+}$, e logo é um conjunto fechado de $E$.

Agora para cada $r \in \mathbb{R}^{+}$,

$$
\{x \in E: p(x) \leq r\}=\cap_{k=1}^{\infty}\left\{x \in E: p_{k}(x) \leq r\right\} .
$$

Logo para cada $r \in \mathbb{R}^{+},\{x \in E: p(x) \leq r\}$ é intersecção de conjuntos fechados, e logo um conjunto fechado.

Assim a imagem inversa de $p$ de qualquer subconjunto fechado de $\mathbb{R}^{+}$é um subconjunto fechado de $E$, portanto, $p$ é contínuo.

Logo, existe $\gamma>0$ tal que $\|x\|<\gamma \Rightarrow p(x)<1$.

Dado $\epsilon>0$ e $x$ qualquer em $E$, então

$$
\left\|\frac{\gamma x}{\|x\|+\epsilon}\right\|=\gamma\left\|\frac{x}{\|x\|+\epsilon}\right\|<\gamma
$$

e

$$
p\left(\frac{\gamma x}{\|x\|+\epsilon}\right)<1 .
$$

Observando-se que para cada $\alpha \in \mathbb{K}, p(\alpha x)=|\alpha| p(x)$ temos que

$$
p\left(\frac{\gamma x}{\|x\|+\epsilon}\right)=\frac{\gamma}{\|x\|+\epsilon} p(x)<1 .
$$

\footnotetext{
${ }^{1}$ Observamos que $p$ é uma seminorma.
} 
Assim, $p(x)<\frac{\|x\|+\epsilon}{\gamma}$. Fazendo-se $\epsilon \rightarrow 0$, temos que $p(x)<\frac{\|x\|}{\gamma}$. Agora tomando-se $M=\frac{1}{\gamma}$, temos que $p(x)<M\|x\|$.

Vamos agora mostrar que $T$ é compacto utilizando o teorema 1.4.11. Por hipótese,

$$
\|T x\| \leq \sum\left|\lambda_{n}\right|\left|g_{n}(x)\right| \leq\|\lambda\|_{\infty} \sum\left|g_{n}(x)\right| \leq\|\lambda\|_{\infty} p(x) \leq\|\lambda\|_{\infty} M\|x\| .
$$

Logo $T$ é contínuo.

Como $\left(\lambda_{n}\right)_{n} \in c_{0}$, dado $\epsilon^{\prime}>0$ existe $n_{0} \in \mathbb{N}$ tal que para $n>n_{0}$, temos que $\sup \left\{\left|\lambda_{k}\right|: k>n_{0}\right\}<\epsilon^{\prime} / M$. Seja $N=\operatorname{kerg}_{1} \cap \cdots \cap \operatorname{kerg}_{n_{o}}$. Para todo $x \in N$ temos que:

$$
\begin{gathered}
\|T(x)\| \leq \sum_{k>n_{0}}\left|\lambda_{k} \| g_{k}(x)\right| \leq \\
\leq \sup \left\{\left|\lambda_{k}\right|: k \geq n_{0}\right\} p(x) \leq \sup \left\{\left|\lambda_{k}\right|: k \geq n_{0}\right\} M\|x\| \leq \epsilon^{\prime}\|x\| .
\end{gathered}
$$

Assim $\left\|\left.T\right|_{N}\right\| \leq \epsilon^{\prime}$. Como $\operatorname{dim}(E / N) \leq n_{0}$, segue do teorema 1.4.11 que $T$ é compacto.

Como conseqüência da proposição 2.4 temos os seguinte corolário.

Corolário 2.5. Sejam $E$ um espaço de Banach e $T: E \rightarrow \ell_{1}$ um operador linear compacto. Então existe uma série $\omega^{*}$ incondicionalmente convergente $\sum h_{n}$ em $E^{\prime}$ tal que para cada $x$ em $E$

$$
T x=\sum h_{n}(x) e_{n}
$$

$\operatorname{com}\left(e_{n}\right)_{n}$ é a base canônica de $\ell_{1}$.

Demonstração: Pela proposição 2.4, (1) $\Rightarrow(2)$, existem $\lambda=\left(\lambda_{n}\right)_{n} \in c_{0}$ e $\sum g_{n}$ incondicionalmente convergente em $E^{\prime}$ tal que $T x=\sum \lambda_{n} g_{n}(x) e_{n}$, para cada $x \in E$, com $\left(e_{n}\right)_{n}$ base canônica de $l_{1}$.

Tomemos $h_{n}=\lambda_{n} g_{n}$ para cada $n \in \mathbb{N}$. Então pela proposição 1.3.5 $\sum h_{n}$ é incondicionalmente convergente e logo pela proposição $1.3 .8, \omega^{*}$ incondicionalmente convergente em $E^{\prime}$. E temos que $T x=\sum h_{n}(x) e_{n}$, para cada $x \in E$.

Observação 2.6. Considere $\left(p_{n}\right)_{n}$ a seqüência de funcionais lineares contínuos definida no exemplo 1.3.7. A série $\sum p_{n}$ é então $\omega^{*}$ incondicionalmente convergente em $\ell_{\infty}=$ 
$\left(\ell_{1}\right)^{\prime}$. Como o operador identidade Id $: \ell_{1} \rightarrow \ell_{1}$ tem uma representação da forma Idx= $\sum p_{n}(x) e_{n}$, com $\left(e_{n}\right)_{n}$ base canônica de $\ell_{1}$, e sabemos que $I d: \ell_{1} \rightarrow \ell_{1}$ não é compacto, podemos ver que a condição do corolário 2.5 não caracteriza operadores lineares compactos em $\ell_{1}$.

Se observamos a demonstração de (3) implica (1) da proposição acima, notamos que se $T$ satisfaz a condição (3), a demonstração é a mesma se colocamos o contradomínio de $T$ qualquer espaço de Banach $F$. Assim temos o seguinte corolário:

Corolário 2.7. Sejam $E$ e $F$ espaços de Banach e $T: E \rightarrow F$ um operador linear. Se existem uma seqüência $\lambda=\left(\lambda_{n}\right)_{n}$ em $c_{0}$ e uma série $\omega^{*}$ incondicionalmente convergente $\sum g_{n}$ em $E^{\prime}$ tais que para cada $x$ em $E$

$$
\|T x\| \leq \sum\left|\lambda_{n}\right|\left|g_{n}(x)\right|
$$

então $T$ é compacto.

A classe de operadores que satisfazem a hipótese do Corolário 2.7 formam um ideal de operadores, como mostra a seguinte proposição. Denotaremos esta classe por $I(E, F)$.

Proposição 2.8. Sejam E, F e G espaços de Banach, $R: E \rightarrow F, S: E \rightarrow F e$ $T: F \rightarrow G$ operadores lineares. Se $R$ e $S \in I(E, F)$, então $R+S \in I(E, F)$. Além disso, se $S \in I(E, F)$ ou $T \in L(F, G)$, então $T \circ S \in I(E, G)$.

Demonstração: Sejam $R$ e $S \in I(E, F) ; \lambda=\left(\lambda_{n}\right)_{n}$ e $\beta=\left(\beta_{n}\right)_{n} \in c_{0} ; \sum g_{n}$ e $\sum h_{n}$ séries $\omega^{*}$ incondicionalmente convergentes em $E^{\prime}$ tais que:

$$
\begin{gathered}
\|R x\| \leq \sum\left|\lambda_{n} \| g_{n}(x)\right| \text { para cada } x \text { em } E \text { e } \\
\|S x\| \leq \sum\left|\beta_{n}\right|\left|h_{n}(x)\right| \text { para cada } x \text { em } E .
\end{gathered}
$$

Seja $\gamma=\left(\gamma_{n}\right)_{n}$ a seguinte seqüência:

$$
\gamma_{n}=\left\{\begin{array}{cc}
\lambda_{\frac{n+1}{2}} & \text { se } n \text { é ímpar, } \\
\beta_{\frac{n}{2}} & \text { se } n \text { é par. }
\end{array}\right.
$$

Seja a série $\sum f_{n}$ com:

$$
f_{n}=\left\{\begin{array}{cc}
g_{\frac{n+1}{2}} & \text { se } n \text { é ímpar, } \\
h_{\frac{n}{2}} & \text { se } n \text { é par. }
\end{array}\right.
$$


Então $\gamma \in c_{0}, \sum f_{n}$ é $\omega^{*}$ incondicionalmente convergente em $E^{\prime}$ e para cada $x \in E$ :

$$
\begin{gathered}
\|(R+S)(x)\| \leq\|R x\|+\|S x\| \leq \\
\leq \sum\left|\lambda_{n}\left\|g_{n}(x)\left|+\sum\right| \beta_{n}|| h_{n}(x)\left|=\sum\right| \gamma_{n}\right\| f_{n}(x)\right| .
\end{gathered}
$$

Assim, $R+S \in I(E, F)$.

Agora se $S \in I(E, F)$, existem $\lambda=\left(\lambda_{n}\right)_{n} \in c_{0}$ e $\sum g_{n}$ série $\omega^{*}$ incondicionalmente convergentes em $E^{\prime}$ tal que:

$$
\|S x\| \leq \sum\left|\lambda_{n} \| g_{n}(x)\right| \text { para cada } x \text { em } E .
$$

Então $T \circ S: E \rightarrow G$ é contínuo e para cada $x$ em $E$

$\|(T \circ S)(x)\| \leq\|T\|\|S x\| \leq\|T\| \sum\left|\lambda_{n}\right|\left|g_{n}(x)\right|=\sum\left|\|T\| \lambda_{n}\right|\left|g_{n}(x)\right|$.

Como $\left(\|T\| \lambda_{n}\right)_{n} \in c_{0}$, segue que $T \circ S \in I(E, G)$.

Se $T \in I(F, G)$, existem $\beta=\left(\beta_{n}\right)_{n} \in c_{0}$ e $\sum h_{n}$ série $\omega^{*}$ incondicionalmente convergentes em $F^{\prime}$ tal que:

$$
\|T y\| \leq \sum\left|\beta_{n} \| h_{n}(y)\right| \text { para cada y em } F .
$$

Então $T \circ S: E \rightarrow G$ é contínuo e para cada $x$ em $E$

$$
\|(T \circ S)(x)\|=\|T(S(x))\| \leq \sum\left|\beta_{n}\left\|h_{n}(S(x))\left|=\sum\right| \beta_{n}\right\|\left(S^{*} \circ h_{n}\right)(x)\right| .
$$

Como $\sum\left|\left(S^{*} \circ h_{n}\right)(x)\right|=\sum\left|h_{n}(S(x))\right|<\infty$ para cada $x$ em $E$, segue que $\sum S^{*} \circ h_{n}$ é $\omega^{*}$ incondicionalmente convergente em $F^{\prime}$ e portanto $T \circ S \in I(E, G)$.

É fácil ver que $I(E, F)$ é um espaço vetorial. Vamos agora definir uma norma em $I(E, F)$.

Proposição 2.9. Considere em $I(E, F)$ a seguinte função $\alpha: I(E, F) \rightarrow \mathbb{R}$, dada por

$$
\alpha(T)=\inf \left[\sup \left\{\sum\left|\lambda_{n}\right|\left|g_{n}(x)\right|:\|x\| \leq 1\right\}\right]
$$


para cada $T \in I(E, F)$, com o infimo tomado sobre $\Gamma=\left\{\lambda=\left(\lambda_{n}\right)_{n} \in c_{0}\right.$ e $\sum g_{n}$ séries $\omega^{*}$ incondicionalmente convergentes em $E^{\prime}$ tais que $\left.\|T x\| \leq \sum\left|\lambda_{n}\right|\left|g_{n}(x)\right|\right\}$.

A função $\alpha($.) é uma norma em $I(E, F)$ e $\|T\| \leq \alpha(T)$ para todo operador $T$ em $I(E, F)$.

Demonstração: Vamos primeiro mostrar que $\|T\| \leq \alpha(T)$ para todo operador $T$ em $I(E, F)$. Seja $T \in I(E, F)$ qualquer. Sejam $\left(\lambda_{n}\right)_{n} \in c_{0}$ e $\sum g_{n}$ uma série $\omega^{*}$ incondicionalmente convergente em $E^{\prime}$ tais que $\|T x\| \leq \sum\left|\lambda_{n}\right|\left|g_{n}(x)\right|$. Como

segue que

$$
\|T\|=\sup _{\|x\| \leq 1}\|T x\| \leq \sup _{\|x\| \leq 1} \sum\left|\lambda_{n}\right|\left|g_{n}(x)\right|
$$

$$
\|T\| \leq \inf _{\Gamma}\left[\sup _{\|x\| \leq 1}\left\{\sum\left|\lambda_{n}\right|\left|g_{n}(x)\right|\right\}\right]
$$

Portanto, $\|T\| \leq \alpha(T)$.

Vamos agora mostrar que $\alpha(T)$ é uma norma. Como $\|T\| \leq \alpha(T)$, temos que $\alpha() \geq$.0 , e que $\alpha(T)=0 \Rightarrow T \equiv 0$. Se $T \equiv 0$, basta tomarmos $\lambda_{n}=0$ e $g_{n} \equiv 0$ para todo $n \in \mathbb{N}$ que teremos

$$
\sup _{\|x\| \leq 1} \sum\left|\lambda_{n}\right|\left|g_{n}\right|=0
$$

e conseqüentemente $\alpha(T)=0$.

Seja $\gamma \in \mathbb{K}$ qualquer. Se $\gamma=0$ então é óbvio que $\alpha(\gamma T)=\gamma \alpha(T)$. Se $\gamma \neq 0$ então para cada $\lambda=\left(\lambda_{n}\right)_{n} \in c_{0}$ e para cada série $\omega^{*}$ incondicionalmente convergente $\sum g_{n} \in E^{\prime}$ tais que

$$
\|T x\| \leq \sum\left|\lambda_{n} \| g_{n}(x)\right|
$$

segue

$$
\|\gamma T x\| \leq|\gamma| \sum\left|\lambda_{n}\left\|g_{n}(x)\left|=\sum\right| \gamma \lambda_{n}\right\| g_{n}(x)\right|
$$

Logo

e assim

$$
\sup _{\|x\| \leq 1}\left\{\sum\left|\gamma \lambda_{n} \| g_{n}(x)\right|\right\}=\sup _{\|x\| \leq 1}\left\{|\gamma| \sum\left|\lambda_{n} \| g_{n}(x)\right|\right\}
$$

$$
\inf _{\Gamma}\left[\sup _{\|x\| \leq 1}\left\{\sum\left|\gamma \lambda_{n} \| g_{n}(x)\right|\right\}\right]=\inf _{\Gamma}\left[\sup _{\|x\| \leq 1}\left\{|\gamma| \sum\left|\lambda_{n} \| g_{n}(x)\right|\right\}\right]=|\gamma| \alpha(T)
$$


Portanto $\alpha(\gamma T) \leq|\gamma| \alpha(T)$. Agora

$$
\alpha(T)=\alpha\left(\frac{\gamma}{\gamma} T\right) \leq \frac{1}{|\gamma|} \alpha(\gamma T) .
$$

Logo

$$
|\gamma| \alpha(T) \leq \alpha(\gamma T)
$$

Portanto $\alpha(\gamma T)=|\gamma| \alpha(T)$.

Sejam $T$ e $S \in I(E, F)$. Vamos agora mostrar que $\alpha(T+S) \leq \alpha(T)+\alpha(S)$. Dado $\epsilon>0$, segue da definição de $\alpha(\cdot)$ que existem $\lambda=\left(\lambda_{n}\right)_{n}$ e $\beta=\left(\beta_{n}\right)_{n} \in c_{0}$, e $\sum g_{n}$ e $\sum h_{n}$ séries $\omega^{*}$ incondicionalmente convergentes em $E^{\prime}$ tais que:

$$
\begin{gathered}
\|T x\| \leq \sum\left|\lambda_{n} \| g_{n}(x)\right|, \\
\|S x\| \leq \sum\left|\beta_{n}\right|\left|h_{n}(x)\right| \text { para cada } x \text { em } E, e \\
\sup _{\|x\| \leq 1}\left\{\sum\left|\lambda_{n} \| g_{n}(x)\right|\right\} \leq \alpha(T)+\epsilon / 2 \\
\sup _{\|x\| \leq 1}\left\{\sum\left|\beta_{n} \| h_{n}(x)\right|\right\} \leq \alpha(S)+\epsilon / 2 .
\end{gathered}
$$

Consideremos $\gamma=\left(\gamma_{n}\right)_{n}$ dada por:

$$
\gamma_{n}=\left\{\begin{array}{cc}
\lambda_{\frac{n+1}{2}} & \text { se } n \text { é ímpar } \\
\beta_{\frac{n}{2}} & \text { se } n \text { é par }
\end{array}\right.
$$

e $\sum f_{n}$ dada por:

$$
f_{n}=\left\{\begin{array}{cc}
g_{\frac{n+1}{2}} & \text { se } n \text { é ímpar } \\
h_{\frac{n}{2}} & \text { se } n \text { é par. }
\end{array}\right.
$$

Então $\gamma \in c_{0}, \sum f_{n}$ é $\omega^{*}$ incondicionalmente convergente em $E^{\prime}$ e para cada $x \in E$, temos que

$$
\|(T+S)(x)\| \leq \sum_{n}\left|\gamma_{n}\left\|f_{n}(x)\left|=\sum_{n}\right| \lambda_{n}\right\| g_{n}(x)\right|+\sum_{n}\left|\beta_{n}\right|\left|h_{n}(x)\right| .
$$

Assim

$$
\sup _{\|x\| \leq 1}\left\{\sum\left|\gamma_{n} \| f_{n}(x)\right|\right\} \leq \sup _{\|x\| \leq 1}\left\{\sum\left|\lambda_{n} \| g_{n}(x)\right|\right\}+\sup _{\|x\| \leq 1}\left\{\sum\left|\beta_{n}\right|\left|h_{n}(x)\right|\right\} \leq
$$




$$
\leq \alpha(T)+\alpha(S)+\epsilon
$$

Agora $\gamma \in c_{0}, \sum f_{n}$ é $\omega^{*}$ incondicionalmente convergente em $E^{\prime}$ e para cada $x \in E$, temos que $\|(T+S)(x)\| \leq \sum_{n}\left|\gamma_{n} \| f_{n}(x)\right|$; e $\alpha(T+S)=\inf \left[\sup \left\{\sum\left|\mu_{n}\right|\left|k_{n}(x)\right|:\|x\| \leq 1\right\}\right]$, com o ínfimo tomado sobre $\Gamma=\left\{\mu=\left(\mu_{n}\right)_{n} \in c_{0}\right.$ e $\sum k_{n}$ séries $\omega^{*}$ incondicionalmente convergentes em $E^{\prime}$ tais que $\left.\|(T+S)(x)\| \leq \sum\left|\mu_{n}\right|\left|k_{n}(x)\right|\right\}$, então $\alpha(T+S) \leq \alpha(T)+\alpha(S)+\epsilon$. Como a desigualdade vale para todo $\epsilon>0$; segue que $\alpha(T+S) \leq \alpha(T)+\alpha(S)$.

O teorema 2.10 seguinte mostra que $\ell_{1}$ é o único espaço de Banach $E$ com base normalizada $\left(u_{n}\right)_{n}$ tal que todo operador linear compacto $T: F \rightarrow E$ de um espaço de Banach $F$ em $E$ tem uma representação da forma $T x=\sum g_{n}(x) u_{n} \operatorname{com} \sum g_{n}$ uma série $\omega^{*}$ incondicionalmente convergente no dual topológico $F^{\prime}$ de $F$. O teorema 2.10 é então usado para dar uma outra caracterização de operadores compactos através de $\ell_{1}$ (corolário 2.11).

Teorema 2.10. Para um espaço de Banach $E$ com base normalizada $\left(u_{n}\right)_{n}$, são equivalentes:

(1) $\left(u_{n}\right)_{n}$ é equivalente a base canônica de $\ell_{1}$.

(2) Para cada operador linear compacto $T: F \rightarrow E, F$ um espaço de Banach, existem uma seqüencia $\lambda=\left(\lambda_{n}\right)_{n}$ em $c_{0}$ e uma série incondicionalmente convergente $\sum g_{n}$ em $F^{\prime}$ tais que para cada y em $F$

$$
T y=\sum \lambda_{n} g_{n}(y) u_{n}
$$

(3) Para cada operador linear compacto $T: F \rightarrow E, F$ um espaço de Banach, existe uma série $\omega^{*}$ incondicionalmente convergente $\sum h_{n}$ em $F^{\prime}$ tal que para cada y em $F$

$$
T y=\sum h_{n}(y) u_{n}
$$

(4) Para cada operador linear compacto $T: E \rightarrow E$, existe uma série $\omega^{*}$ incondicionalmente convergente $\sum h_{n}$ em $E^{\prime}$ tal que para cada $x$ em $E$

$$
T x=\sum h_{n}(x) u_{n}
$$


Demonstração: $(1) \Rightarrow(2)$ Seja $\left(e_{n}\right)_{n}$ a base canônica de $l_{1}$. Como por hipótese $\left(e_{n}\right)_{n}$ e $\left(u_{n}\right)_{n}$ são equivalentes, pela proposição 1.3 .25 existe um isomorfismo $S: E \rightarrow l_{1}$ com $S\left(u_{n}\right)=e_{n}$ para cada $n$.

Então $S \circ T: F \rightarrow l_{1}$ é um operador compacto e pela proposição 2.4, existem $\lambda=$ $\left(\lambda_{n}\right)_{n} \in c_{0}$ e $\sum g_{n}$ incondicionalmente convergente em $F^{\prime}$ tais que para cada $y \in F$, $(S \circ T)(y)=\sum \lambda_{n} g_{n}(y) e_{n}$.

Logo,

$$
T(y)=S^{-1}\left(\sum \lambda_{n} g_{n}(y) e_{n}\right)=\sum \lambda_{n} g_{n}(y) S^{-1}\left(e_{n}\right)=\sum \lambda_{n} g_{n}(y) u_{n} .
$$

(2) $\Rightarrow(3)$ Por hipótese, para cada operador linear compacto $T: F \rightarrow E$, existem uma seqüência $\lambda=\left(\lambda_{n}\right)_{n}$ em $c_{0}$ e uma série incondicionalmente convergente $\sum g_{n}$ em $F^{\prime}$ tais que para cada $y$ em $F$

$$
T y=\sum \lambda_{n} g_{n}(y) u_{n}
$$

Para cada $n \in \mathbb{N}$, seja $h_{n}=\lambda_{n} g_{n}$. Para cada $y \in F, T y=\sum h_{n}(y) u_{n}$ e das proposições 1.3 .5 e 1.3.8, temos que $\sum h_{n}$ é $\omega^{*}$ incondicionalmente convergente.

(3) $\Rightarrow$ (4) é imediato.

(4) $\Rightarrow$ (1) Vamos mostrar que $\left(e_{n}\right)_{n}$ e $\left(u_{n}\right)_{n}$ são equivalentes. Seja $\left(f_{n}\right)_{n}$ a seqüência de coeficientes funcionais associada à $\left(u_{n}\right)_{n}$. Como $\left(u_{n}\right)_{n}$ é uma base normalizada, dado $\alpha=\sum \alpha_{n} e_{n}$ em $\ell_{1}$, temos que

$$
\sum\left\|\alpha_{n} u_{n}\right\|=\sum\left|\alpha_{n}\right|\left\|u_{n}\right\|=\sum\left|\alpha_{n}\right|<\infty
$$

então $\sum \alpha_{n} u_{n}$ é absolutamente convergente em $E$ e segue da proposição 1.3.4 que a série é convergente em $E$.

Considere $x=\sum \beta_{n} u_{n}$ em $E$, como $\left(u_{n}\right)_{n}$ é base de Schauder para $E$, pela unicidade da representação, $x=\sum f_{n}(x) u_{n}$. Como $\left\|e_{n}\right\|=1$, temos que

$$
\sum\left\|f_{n}(x) e_{n}\right\|=\sum\left|f_{n}(x)\right| .
$$

Como $\ell_{1}$ é Banach, pela proposição 1.3.4, para mostrar a implicação (1), é suficiente mostrar que $\sum\left|f_{n}(x)\right|$ converge. Para tal, vamos definir um operador linear compacto $T: E \rightarrow E$ e utilizar as hipóteses de (4). 
Pelo teorema 1.3.15, para cada $x=\sum_{k=1}^{\infty} f_{k}(x) u_{k}$ em $E$,

$$
\|x\|_{\left(x_{n}\right)}=\sup _{n}\left\|\sum_{k=1}^{n} f_{k}(x) u_{k}\right\|
$$

define uma norma em $E$ equivalente à norma original.

Fixados $n \leq m$, para cada $x=\sum_{k=1}^{\infty} f_{k}(x) u_{k}$ em $E$, temos que

$$
\sum_{k=n}^{m} f_{k}(x) u_{k}=\sum_{k=1}^{m} f_{k}(x) u_{k}-\sum_{k=1}^{n-1} f_{k}(x) u_{k},
$$

$\log 0$

$$
\left\|\sum_{k=n}^{m} f_{k}(x) u_{k}\right\| \leq\left\|\sum_{k=1}^{m} f_{k}(x) u_{k}\right\|+\left\|\sum_{k=1}^{n-1} f_{k}(x) u_{k}\right\| .
$$

Assim

$\sup \left\{\left\|\sum_{k=n}^{m} f_{k}(x) u_{k}\right\|: n \leq m<\infty\right\} \leq \sup \left\{\left\|\sum_{k=1}^{m} f_{k}(x) u_{k}\right\|+\left\|\sum_{k=1}^{n-1} f_{k}(x) u_{k}\right\|: n \leq m<\infty\right\} \leq$

$$
\leq \sup _{m}\left\|\sum_{k=1}^{m} f_{k}(x) u_{k}\right\|+\sup _{m}\left\|\sum_{k=1}^{m-1} f_{k}(x) u_{k}\right\| \leq 2\|x\|_{\left(x_{n}\right)} .
$$

Seja $\lambda_{1} \geq \lambda_{2} \geq \cdots$ uma seqüência não-crescente de números positivos convergindo para zero. De

$$
\sum_{k=n}^{m} \lambda_{k} f_{k}(x) u_{k}=\sum_{k=n}^{m-1}\left[\left(\lambda_{k}-\lambda_{k+1}\right) \sum_{j=n}^{k} f_{j}(x) u_{j}\right]+\lambda_{m} \sum_{k=n}^{m} f_{k}(x) u_{k},
$$

segue que,

$$
\begin{gathered}
\left\|\sum_{k=n}^{m} \lambda_{k} f_{k}(x) u_{k}\right\| \leq\left\|\sum_{k=n}^{m-1}\left[\left(\lambda_{k}-\lambda_{k+1}\right) \sum_{j=n}^{k} f_{j}(x) u_{j}\right]\right\|+\left\|\lambda_{m} \sum_{k=n}^{m} f_{k}(x) u_{k}\right\| \leq \\
\leq \sum_{k=n}^{m-1}\left(\lambda_{k}-\lambda_{k+1}\right)\left\|\sum_{j=n}^{k} f_{j}(x) u_{j}\right\|+\lambda_{m}\left\|\sum_{k=n}^{m} f_{k}(x) u_{k}\right\| \leq \\
\leq\left[\sum_{k=n}^{m-1}\left(\lambda_{k}-\lambda_{k+1}\right)+\lambda_{m}\right] \sup \left\{\left\|\sum_{k=n}^{m} f_{k}(x) u_{k}\right\|: n \leq m<\infty\right\} \leq
\end{gathered}
$$




$$
\leq \lambda_{n} 2\|x\|_{\left(x_{n}\right)} .
$$

Logo, temos que

$$
\left\|\sum_{k=n}^{m} \lambda_{k} f_{k}(x) u_{k}\right\| \leq \lambda_{n} 2\|x\|_{\left(x_{n}\right)}
$$

para cada $x=\sum_{k=1}^{\infty} f_{k}(x) u_{k}$ em $E$.

Como a seqüência $\left(\lambda_{n}\right)_{n}$ converge a zero, segue que a série $\sum \lambda_{n} f_{n}(x) u_{n}$ é Cauchy somável e logo, pela proposição 1.3.2, incondicionalmente convergente e portanto convergente.

Assim podemos definir o operador linear $T: E \rightarrow E$ dado por

$$
\begin{gathered}
T x=\sum \lambda_{n} f_{n}(x) u_{n} \\
\operatorname{com}\|T(x)\| \leq \lambda_{1} 2\|x\|_{\left(x_{n}\right)} .
\end{gathered}
$$

Vamos mostrar que $T$ é compacto. Como $\left(\lambda_{n}\right)_{n}$ converge a zero, dado $\epsilon>0$, existe $n_{0} \in \mathbb{N}$ tal que para $n>n_{0}$, temos que $\left|\lambda_{n}\right|<\epsilon / 2$. Seja $N=\operatorname{ker} f_{1} \cap \cdots \cap k e r f_{n_{0}}$. Para todo $x \in N$, temos que

$$
\|T x\| \leq \lambda_{n_{0}+1} 2\|x\|_{\left(x_{n}\right)} \leq \epsilon\|x\|_{\left(x_{n}\right)} .
$$

Como $\operatorname{dim}(E / N)=n_{0}$ segue do teorema 1.4.11 que $T$ é compacto. Portanto, pela hipótese, existe uma série $\omega^{*}$ incondicionalmente convergente $\sum h_{n}$ em $E^{\prime}$ tal que para cada $x$ em $E$

$$
T x=\sum h_{n}(x) u_{n}
$$

Mas então, como $\left(u_{n}\right)_{n}$ é base de Schauder para $E$, pela unicidade da representação, segue que

$$
\lambda_{n} f_{n}(x)=h_{n}(x)
$$

para cada $x$ em $E$ e

$$
\sum\left|\lambda_{n}\right|\left|f_{n}(x)\right| \leq \sum\left|h_{n}(x)\right|<\infty
$$

para cada $x$ em $E$. Pelo lema 1.3.9, segue que $\sum\left|f_{n}(x)\right|<\infty$ para cada $x$ em $E$ e segue (1). 
Parte da demonstração da implicação $(4) \Rightarrow(1)$ do teorema 2.10 está baseada em uma modificação da demonstração de um resultado apresentado em [15], (ver proposição 1.3.10). Utilizaremos também este resultado na demonstração do próximo corolário.

Se a base é incondicional temos a seguinte versão para o teorema 2.10 .

Corolário 2.11. Para um espaço de Banach $E$ com base incondicional normalizada $\left(u_{n}\right)_{n}$ são equivalentes:

(1) $\left(u_{n}\right)_{n}$ é equivalente a base canônica de $\ell_{1}$.

(2) Todo operador linear compacto $T: c_{0} \rightarrow E$ tem uma representação da forma

$$
T \lambda=\sum g_{n}(\lambda) u_{n}, \text { para cada } \lambda \in c_{0}
$$

com $\sum g_{n}$ uma série $\omega^{*}$ incondicionalmente convergente em $\ell_{1}=\left(c_{0}\right)^{\prime}$.

Demonstração: $(1) \Rightarrow(2)$ segue do teorema 2.10 considerando $F=c_{0}$.

(2) $\Rightarrow$ (1) Vamos mostrar que $\left(e_{n}\right)_{n}$ e $\left(u_{n}\right)_{n}$ são equivalentes. Seja $\left(f_{n}\right)_{n}$ a seqüência de coeficientes funcionais associada à $\left(u_{n}\right)_{n}$. Como $\left(u_{n}\right)_{n}$ é normalizada, dado $\alpha=\sum \alpha_{n} e_{n}$ em $\ell_{1}$, temos que

$$
\sum\left\|\alpha_{n} u_{n}\right\|=\sum\left|\alpha_{n}\right|\left\|u_{n}\right\|=\sum\left|\alpha_{n}\right|<\infty
$$

então $\sum \alpha_{n} u_{n}$ é absolutamente convergente em $E$, segue da proposição 1.3.4 que a série converge em $E$.

Dado $x=\sum \beta_{n} u_{n}$ em $E$, como $\left(u_{n}\right)_{n}$ é base de Schauder para $E$, pela unicidade da representação, $x=\sum f_{n}(x) u_{n}$. E como $\left\|e_{n}\right\|=1$, temos que

$$
\sum\left\|f_{n}(x) e_{n}\right\|=\sum\left|f_{n}(x)\right|
$$

Como $\ell_{1}$ é Banach, pela proposição 1.3.4, para mostrar a implicação (1), é suficiente mostrar que $\sum\left|f_{n}(x)\right|$ converge. Para tal, vamos definir um operador linear compacto $T: c_{0} \rightarrow E$ e utilizar as hipóteses de (2).

Como $\left(u_{n}\right)_{n}$ é uma base incondicional, pelo teorema 1.3.23 podemos assumir que para 
cada $x=\sum_{k=1}^{\infty} f_{k}(x) u_{k}$ em $E$

$$
\|x\|_{(u)}=\sup \left\{\left\|\sum_{k \in A}\right\| f_{k}(x) u_{k} \|: A \text { é um subconjunto finito de } \mathbb{N}\right\}
$$

define uma norma equivalente à norma original de $E$.

Portanto, pela proposição 1.3 .10 segue que para cada $\lambda=\left(\lambda_{n}\right)_{n}$ em $c_{0}$ e para cada $x=\sum f_{n}(x) u_{n}$ em $E$, fixados $n \leq m \in \mathbb{N}$, temos que

$$
\left\|\sum_{k=n}^{m} \lambda_{k} f_{k}(x) u_{k}\right\| \leq 4 \sup \left\{\left|\lambda_{k}\right|: n \leq k \leq m\right\}\|x\|_{(u)} .
$$

Como $\left(\lambda_{n}\right)_{n} \in c_{0}$, dado $\epsilon>0$, existe $n_{0} \in \mathbb{N}$ tal que para $n \geq n_{0}$ temos que $\sup \left\{\left|\lambda_{k}\right|: k>n_{0}\right\}<\epsilon / 4\|x\|_{(u)}$.

Assim, de 2.5, temos que a série $\sum \lambda_{n} f_{n}(x) u_{n}$ é Cauchy somável e logo, pela proposição 1.3.2, incondicionalmente convergente, para cada $\lambda=\left(\lambda_{n}\right)_{n}$ em $c_{0}$ e cada $x=\sum_{k=1}^{\infty} f_{k}(x) u_{k}$ em $E$.

Fixe $x$ em $E$ e seja $\mu_{1} \geq \mu_{2} \geq \cdots$ uma seqüência não-crescente de números positivos convergindo para zero. Seja $T: c_{0} \rightarrow E$ o operador linear dado por

$$
T \lambda=\sum \lambda_{n} \mu_{n} f_{n}(x) u_{n}
$$

para cada $\lambda=\left(\lambda_{n}\right)_{n}$ em $c_{0}$.

Novamente utilizando a proposição 1.3.10, temos que:

$$
\left\|\sum_{n=1}^{m} \lambda_{n} \mu_{n} f_{n}(x) u_{n}\right\| \leq 4 \sup \left\{\left|\lambda_{n} \mu_{n}\right|: 1 \leq n \leq m\right\}\|x\|_{(u)} .
$$

Logo, para cada $\lambda=\left(\lambda_{n}\right)_{n} \in c_{0}$

$$
\|T \lambda\|=\left\|\sum_{n=1}^{\infty} \lambda_{n} \mu_{n} f_{n}(x) u_{n}\right\|=\lim _{m \rightarrow \infty}\left\|\sum_{n=1}^{m} \lambda_{n} \mu_{n} f_{n}(x) u_{n}\right\| \leq 4\|\lambda\|_{\infty} \mu_{1}\|x\|_{(u)} .
$$

Assim, temos que $T$ é contínuo. Vamos mostrar que $T$ é compacto utilizando o teorema 1.4.11.

Como $\mu_{1}, \ldots, \mu_{n}, \ldots$ é uma seqüência que converge a zero, dado $\epsilon^{\prime}>0$, existe $n_{1} \in \mathbb{I}$ tal que para $n>n_{1}$, temos que $\mu_{n}<\epsilon^{\prime} / 4\|x\|_{(u)}$. 
Seja $N=\left\{\lambda=\left(\lambda_{n}\right)_{n} \in c_{0}: \lambda_{k}=0\right.$ para $\left.k \leq n_{1}\right\}$. Para todo $\lambda \in N$, pela proposição 1.3.10, temos que:

$$
\|T \lambda\|=\left\|\sum_{k>n_{1}} \lambda_{k} \mu_{k} f_{k}(x) u_{k}\right\| \leq 4\|\lambda\|_{\infty} \mu_{n_{1}+1}\|x\|_{(u)} \leq \epsilon^{\prime}\|x\|_{(u)} .
$$

Denotando-se por $\left(e_{n}\right)_{n}$ a base canônica de $c_{0}, N=\left[e_{n_{1}+1}, e_{n_{1}+2}, \ldots\right]$. Logo, $\operatorname{dim}(E / N)=n_{1}<\infty$. Portanto, pelo teorema 1.4.11, T é compacto.

Assim, pela hipótese existe uma série $\omega^{*}$ incondicionalmente convergente $\sum g_{n}$ em $\ell_{1}$ tal que $T \lambda=\sum g_{n}(\lambda) u_{n}$ para cada $\lambda=\left(\lambda_{n}\right)_{n}$ em $c_{0}$. Mas como $\left(u_{n}\right)_{n}$ é base de Schauder de $E$, segue que

$$
\lambda_{n} \mu_{n} f_{n}(x)=g_{n}(\lambda)
$$

para cada $\lambda=\left(\lambda_{n}\right)_{n}$ em $c_{0}$ e para cada $n \in \mathbb{N}$. Como $\sum g_{n}$ é $\omega^{*}$ incondicionalmente convergente

$$
\sum\left|\lambda_{n}\right|\left|\mu_{n}\right|\left|f_{n}(x)\right| \leq \sum\left|g_{n}(\lambda)\right|<\infty
$$

para cada $\lambda$ em $c_{0}$, então pelo lema 1.3 .9 ,

$$
\sum\left|\mu_{n}\right|\left|f_{n}(x)\right|<\infty \text { e } \sum\left|f_{n}(x)\right|<\infty
$$

Terminamos este capítulo apresentando a seguinte proposição.

Proposição 2.12. Sejam E e F espaços de Banach, e $T: E \rightarrow F$ um operador linear contínuo. Então são equivalentes:

(1) Existem uma seqüência $\lambda=\left(\lambda_{n}\right)_{n}$ em $c_{0}$, uma série incondicionalmente convergente $\sum g_{n}$ em $E^{\prime}$ e uma seqüência limitada $\left(y_{n}\right)_{n}$ em $F$ tais que, para cada $x$ em $E$,

$$
T x=\sum \lambda_{n} g_{n}(x) y_{n}
$$

(2) Existem uma seqüência $\lambda=\left(\lambda_{n}\right)_{n}$ em $c_{0}$, uma série $\omega^{*}$ incondicionalmente convergente $\sum g_{n}$ em $E^{\prime}$ e uma seqüência limitada $\left(y_{n}\right)_{n}$ em $F$ tais que, para cada $x$ em $E$,

$$
T x=\sum \lambda_{n} g_{n}(x) y_{n} .
$$

(3) Existem operadores lineares compactos $P: E \rightarrow \ell_{1}$ e $Q: \ell_{1} \rightarrow F$ tais que $T=Q \circ P$. 
Demonstração: $(1) \Rightarrow(2)$ segue da proposição 1.3.8.

(2) $\Rightarrow$ (3). Pela hipótese, $T$ tem uma representação da forma

$$
T x=\sum \lambda_{n} g_{n}(x) y_{n}
$$

com $\lambda=\left(\lambda_{n}\right)_{n}$ seqüência em $c_{0}, \sum g_{n}$ série $\omega^{*}$ incondicionalmente convergente em $E^{\prime}$ e $\left(y_{n}\right)_{n}$ seqüência limitada em $F$.

Para cada $n \in \mathbb{N}$, se $\lambda_{n} \in \mathbb{C}$, sejam $\gamma_{n}$ tal que $\gamma_{n}^{2}=\lambda_{n}$, e $h_{n}=g_{n}$; se $\lambda_{n} \in \mathbb{R}$, sejam $\gamma_{n}=\sqrt{\left|\lambda_{n}\right|}$ e $h_{n}=\left\{\begin{array}{cc}g_{n} & \text { se } \lambda_{n} \geq 0, \\ -g_{n} & \text { se } \lambda_{n}<0 .\end{array}\right.$

Então $\left(\gamma_{n}\right)_{n} \in c_{0}, \sum h_{n}$ é uma série $\omega^{*}$ incondicionalmente convergente em $E^{\prime}$ e $T x=$ $\sum \gamma_{n} \gamma_{n} h_{n}(x) y_{n}$, para cada $x \in E$.

Seja $P: E \rightarrow \ell_{1}$ um operador linear definido por $P x=\sum \gamma_{n} h_{n}(x) e_{n}$ para cada $x$ em $E, \operatorname{com}\left(e_{n}\right)_{n}$ base canônica de $l_{1}$. De

$$
\sum\left\|\gamma_{n} h_{n}(x) e_{n}\right\|=\sum\left|\gamma_{n}\right|\left|h_{n}(x)\right| \leq\|\gamma\|_{\infty} \sum\left|h_{n}(x)\right|
$$

para cada $x \in E$, temos que o operador $P$ está bem definido, pois a série $\sum \gamma_{n} h_{n}(x) e_{n}$ converge absolutamente em $\ell_{1}$, e é contínuo.

Segue da proposição 2.4 que $P$ é compacto.

Seja $Q: \ell_{1} \rightarrow F$ um operador linear definido por $Q \mu=\sum \gamma_{n} \mu_{n} y_{n}$ para cada $\mu=\left(\mu_{n}\right)_{n}$ em $\ell_{1}$. Como $\left(y_{n}\right)_{n}$ é limitada, existe $M \in \mathbb{R}$ tal que $\left\|y_{n}\right\| \leq M$ para todo $n \in \mathbb{N}$. Para cada $\mu=\left(\mu_{n}\right)_{n} \in \ell_{1}$, temos que

$$
\begin{gathered}
\sum\left\|\gamma_{n} \mu_{n} y_{n}\right\| \leq \\
\leq M \sum\left|\mu_{n}\right|\left|\gamma_{n}\right|=M\|\gamma\|_{\infty} \sum\left|\mu_{n}\right|=M\|\gamma\|_{\infty}\|\mu\|_{1}
\end{gathered}
$$

e assim a série $\sum \gamma_{n} \mu_{n} y_{n}$ converge, isto significa que $Q$ está bem definido e é contínuo.

Vamos agora usar o teorema 1.4.11 para mostrar que $Q$ é compacto.

Como $\gamma \in c_{0}$, dado $\epsilon>0$, existe $n_{0} \in \mathbb{N}$ tal que para $n>n_{0}$ temos que $\left|\gamma_{n}\right|<\epsilon / M$.

Seja $N=\left\{\left(\mu_{n}\right)_{n} \in \ell_{1}: \mu_{n}=0\right.$ para $\left.n \leq n_{0}\right\}$. Para todo $\mu=\left(\mu_{n}\right)_{n} \in N$, temos que

$$
\|Q \mu\|=\sum_{k>n_{0}}\left|\gamma_{k}\right|\left|\mu_{k}\right|\left\|y_{k}\right\| \leq
$$




$$
\leq M \sum_{k>n_{0}}\left|\gamma_{k}\right|\left|\mu_{k}\right| \leq M \sup _{k>n_{0}}\left|\gamma_{k}\right| \sum_{k>n_{0}} \mu_{k} \leq M \frac{\epsilon}{M}\|\mu\|_{1}=\epsilon\|\mu\|_{1} .
$$

Temos que $N=\left[e_{n_{0}+1}, e_{n_{0}+2}, \ldots\right],\left(\left(e_{n}\right)_{n}\right.$ base canônica de $\left.\ell_{1}\right)$. Logo $\operatorname{dim}\left(\ell_{1} / N\right)=$ $n_{0}<\infty$. Portanto segue do teorema 1.4.11 que $Q$ também é compacto.

Agora para cada $x \in E$, temos que $(Q \circ P)(x)=\sum \lambda_{n} g_{n}(x) y_{n}$, isto é, $Q \circ P=T$. $(3) \Rightarrow(1)$. Suponha que existam $P: E \rightarrow \ell_{1}$ e $Q: \ell_{1} \rightarrow F$ operadores compactos tais que $Q \circ P=T$.

Pela proposição 2.4, existem uma seqüência $\lambda=\left(\lambda_{n}\right)$ em $c_{0}$ e uma série incondicionalmente convergente $\sum g_{n}$ em $E^{\prime}$ tal que para cada $x$ em $E$

$$
P x=\sum \lambda_{n} g_{n}(x) e_{n}
$$

$\operatorname{com}\left(e_{n}\right)_{n}$ base unitária canônica de $\ell_{1}$.

Tome $y_{n}=Q\left(e_{n}\right)$ para cada $n \in \mathbb{N}$. Como $Q$ é compacto, $\left(y_{n}\right)_{n}$ é limitada em $F$.

Por hipótese, $T=Q \circ P$, então, para cada $x \in E$,

$$
\begin{gathered}
T(x)=(Q \circ P)(x)=Q\left(\sum \lambda_{n} g_{n}(x) e_{n}\right)= \\
=\sum \lambda_{n} g_{n}(x) Q\left(e_{n}\right)=\sum \lambda_{n} g_{n}(x) y_{n} .
\end{gathered}
$$




\section{Capítulo 3}

\section{Espaços de Banach $F$ para os quais $L(C(\Omega), F)=K(C(\Omega), F)$}

O objetivo deste capítulo é estudar algumas caracterizações dos espaços de Banach $F$ para os quais todos os operadores contínuos de $C(\Omega)$ em $F$ são compactos, com $\Omega$ um espaço de Hausdorff compacto.

Os resultados que apresentamos neste capítulo foram estudados no texto científico de Ansari [2].

\subsection{Caracterizações para Espaços Compactos Dispersos}

Nesta seção, $\Omega$ denotará um espaço de Hausdorff compacto disperso (ver definição 1.1.20). Neste caso mostramos que todos os operadores contínuos de $C(\Omega)$ em $F$ são compactos se, e somente se, todos os operadores de um subespaço fechado de $c_{0}$ em $F$ são compactos se, e somente se, $F$ não contém cópia de $c_{0}$.

Começamos a seção mostrando que se $E$ é um subespaço fechado de $c_{0}$, de dimensão infinita, e $F$ é um espaço de Banach, então todos os operadores contínuos de $E$ em $F$ são compactos se, e somente se, $F$ não contém cópia de $c_{0}$. 
Proposição 3.1.1. Sejam E um subespaço fechado de $c_{0}$, de dimensão infinita, e $F$ um espaço de Banach. Então as seguintes afirmações são equivalentes:

(1) $L(E, F)=K(E, F)$.

(2) F não contém cópia de $c_{0}$.

Demonstração: (1) implica (2) Suponhamos por contradição que $c_{0} \hookrightarrow F$. Então existem um subespaço $Z, Z \subseteq F$ e $T: c_{0} \rightarrow Z$ um isomorfismo.

Consideremos $\left.T\right|_{E}: E \rightarrow Z$. Assim, $\left.T\right|_{E} \in L(E, F)=K(E, F)$ e conseqüentemente $\overline{\left.T\right|_{E}\left(B_{E}\right)}$ é um subconjunto compacto de $F$.

Agora, como $\left.T\right|_{E}$ é um isomorfismo de $E$ sobre $T(E)$, segue que $\left(\left.T\right|_{E}\right)^{-1}$ é contínuo e $\overline{\left.T\right|_{E}\left(B_{E}\right)}=\left.T\right|_{E}\left(B_{E}\right)$. Assim, $\left(\left.T\right|_{E}\right)^{-1}\left(\left.T\right|_{E}\left(B_{E}\right)\right)=B_{E}$ é um subconjunto compacto de $E$, absurdo uma vez que a dimensão de $E$ é infinita.

(2) implica (1) Suponhamos que $c_{0} \psi F$. Sejam $T \in L(E, F)$ e $\left(x_{n}\right)_{n}$ uma seqüência limitada em $E$. Vamos mostrar que $\left(T x_{n}\right)_{n}$ admite uma subseqüência convergente em $F$ e conseqüentemente $T \in K(E, F)$.

Como $E$ é um subespaço de $c_{0}$ e $\ell_{1} \triangleleft c_{0}$, temos que $\ell_{1} \psi \nrightarrow E$. Logo, pelo teorema 1.2.14, $\left(x_{n}\right)_{n}$ admite uma subseqüência fracamente de Cauchy. Podemos assumir, sem perda de generalidade que $\left(x_{n}\right)_{n}$ é fracamente de Cauchy.

Seja $y_{m, n}=x_{n}-x_{m}$. Então, $\left(y_{m, n}\right)_{m, n}$ é uma rede que converge fracamente a zero. Como $T$ é contínuo, pelo teorema 1.2.10, $\left(T y_{m, n}\right)_{m, n}$ também converge fracamente a zero.

Afirmamos que $\left\|T y_{m, n}\right\| \rightarrow 0$. Vamos supor por absurdo que $\left\|T y_{m, n}\right\| \not \rightarrow 0$

Então existem $\epsilon>0$ e seqüências $\left(m_{k}\right)_{k}$ e $\left(n_{k}\right)_{k}$ de números naturais tais que $m_{k}>$ $m_{k-1} \geq k-1, n_{k}>n_{k-1} \geq k-1 \mathrm{e}\left\|T y_{m_{k}, n_{k}}\right\|>\epsilon, \forall k$. Então, pelo teorema 1.3.26, $\left(T y_{m_{k}, n_{k}}\right)_{k}$ admite uma subseqüência que é uma seqüência básica em $F$. Podemos assumir, sem perda de generalidade que $T\left(y_{m_{k}, n_{k}}\right)_{k}$ é uma seqüência básica em $F$

Como $\left(y_{m_{k}, n_{k}}\right)_{k}$ converge fracamente a zero, inf $\left\|y_{m_{k}, n_{k}}\right\| \geq \epsilon>0$ e $\left(y_{m_{k}, n_{k}}\right)_{k} \subseteq E \subseteq$ $c_{0}$, pelo Princípio de Seleção de Bessaga-Pelczynski (teorema 1.3.29), $\left(y_{m_{k}, n_{k}}\right)_{k}$ admite uma subseqüência que é uma seqüência básica equivalente a uma seqüência de blocos da base canônica de $c_{0}$. Podemos assumir, sem perda de generalidade, que $\left(y_{m_{k}, n_{k}}\right)_{k}$ é uma seqüência básica equivalente a uma seqüência de blocos da base canônica de $c_{0}$. Assim, 
pelo teorema 1.3.28, temos que o espaço fechado gerado por $\left(y_{m_{k}, n_{k}}\right)_{k}$ é isomorfo a $c_{0}$.

Agora como $T$ é um operador linear contínuo, para toda seqüência de escalares $\left(a_{k}\right)$, $\sum a_{k} y_{m_{k}, n_{k}}$ converge se, e somente se, $\sum a_{k} T y_{m_{k}, n_{k}}$ converge. Logo as seqüências $\left(y_{m_{k}, n_{k}}\right)_{k}$ $\mathrm{e}\left(T y_{m_{k}, n_{k}}\right)_{k}$ são equivalentes.

Assim, pela proposição 1.3 .25 , os espaços fechados gerados por $\left(y_{m_{k}, n_{k}}\right)_{k}$ e por $T\left(y_{m_{k}, n_{k}}\right)_{k}$ são isomorfos. Então o subespaço de $F, \overline{\left[T\left(y_{m_{k}, n_{k}}\right): k \in \mathbb{N}\right]}$, é isomorfo a $c_{0}$, contradizendo a hipótese inicial.

Corolário 3.1.2. Para um espaço de Banach $F$, as seguintes afirmações são equivalentes:

(1) Para cada conjunto infinito $\Omega$ que seja um espaço de Hausdorff compacto disperso, então $L(C(\Omega), F)=K(C(\Omega), F)$.

(2) Para algum conjunto infinito $\Omega$ que seja um espaço de Hausdorff compacto disperso, então $L(C(\Omega), F)=K(C(\Omega), F)$.

(3) F não contém cópia de $c_{0}$.

(4) Para cada subespaço fechado $E$ de dimensão infinita de $c_{0}$, temos que $L(E, F)=$ $K(E, F)$.

(5) Para algum subespaço fechado $E$ de dimensão infinita de $c_{0}$, temos que $L(E, F)=$ $K(E, F)$.

Demonstração: (1) implica (2) é imediato.

(2) implica (3) Suponhamos por contradição que $c_{0} \hookrightarrow F$. Então existem $Z$ um subespaço de $F$ e $R: c_{0} \rightarrow Z$ um isomorfismo.

Como $\Omega$ é um conjunto infinito e é um espaço compacto disperso, segue do teorema 1.1.21 que existe um subespaço $M$ complementado de $C(\Omega)$ que é isométrico a $c_{0}$.

Sejam $P$ a projeção contínua de $C(\Omega)$ sobre $M, S$ a isometria de $M$ sobre $c_{0}$ e $T=R \circ S$ (um isomorfismo de $M$ em $Z$ ). Então $T \circ P \in L(C(\Omega), Z)$.

Para cada $n \in \mathbb{N}$, sejam $x_{n}=S^{-1}\left(e_{n}\right)$ e $z_{n}=R\left(e_{n}\right)$, com $\left(e_{n}\right)_{n}$ a base canônica de $c_{0}$. Como $P$ é uma projeção de $C(\Omega)$ em $M$ e $\left(x_{n}\right)_{n} \in M$, segue que $P\left(x_{n}\right)=x_{n}$ para cada $n \in \mathbb{N}$. Assim, $(T \circ P)\left(x_{n}\right)=R\left(S\left(P\left(x_{n}\right)\right)\right)=R\left(S\left(x_{n}\right)\right)=R\left(e_{n}\right)=z_{n}$ para cada $n \in \mathbb{N}$.

Como $\left(e_{n}\right)_{n}$ é uma seqüência limitada em $c_{0}$ que não admite subseqüências convergentes, $S^{-1}$ é uma isometria e $R$ é um isomorfismo, segue que $\left(x_{n}\right)_{n}$ e $\left(z_{n}\right)_{n}$ são seqüências 
limitadas em $M$ e $Z$ respectivamente, que não admitem subseqüências convergentes. Logo $T \circ P \notin K(C(\Omega), Z)$, contradizendo a hipótese inicial.

(3) implica (1) Sejam $\Omega$ um conjunto infinito, que é um espaço de Hausdorff disperso, e $T \in L(C(\Omega), F)$. Como $c_{0} \nrightarrow F$, segue do teorema 1.4.27 que o operador $T$ é fracamente compacto. Então segue do teorema de Gantmacher, teorema 1.4.26, $T^{*}: F^{\prime} \rightarrow C(\Omega)^{\prime}$ é fracamente compacto.

Pelo teorema 1.1.23, $C(\Omega)^{\prime}$ é isomorfo a $\ell_{1}(\Omega)$. Pela proposição 1.2.13, $\ell_{1}(\Omega)$ tem a propriedade de Schur. Logo, pela proposição 1.4.25, T* é compacto. Portanto pelo teorema de Schauder (teorema 1.4.9), segue que $T$ é compacto.

As equivalências (3) $\Leftrightarrow(4) \Leftrightarrow(5)$ seguem diretamente da proposição anterior (proposição 3.1.1).

As equivalências $(1) \Leftrightarrow(2) \Leftrightarrow(3)$ deste corolário foram apresentadas em Ansari [2], mas já tinham sido provadas anteriormente por Pelczynski [19], pág. 385, proposição 2.

Corolário 3.1.3 (Pitt). Para $1 \leq p<\infty$, temos que $L\left(c_{0}, \ell_{p}\right)=K\left(c_{0}, \ell_{p}\right)$.

Demonstração: Como $c_{0} \leftrightarrow \ell_{p}$ então pela proposição 3.1 .1 , temos que $L\left(c_{0}, \ell_{p}\right)=$ $K\left(c_{0}, \ell_{p}\right)$.

\subsection{Seqüências $l_{p}^{\omega}(F)$}

Esta seção fornece uma caracterização completa de todos os espaços de Banach $F$, em termos de seqüências $l_{p}^{\omega}(F)$, para os quais $L(E, F)=K(E, F)$, com $E=c_{0}$ ou $\ell_{p}$ $(1 \leq p<\infty)$. No que segue apresentamos a definição do espaço de seqüências $l_{p}-$ fracas em um espaço de Banach.

Definição 3.2.1. (Seqüências $l_{p}$ fracas)

Sejam E um espaço de Banach e $1 \leq p<\infty$. Uma seqüência $\left(x_{n}\right)_{n}$ de elementos de $E$ é chamada seqüência $l_{p}-$ fraca, se para todo $f \in E^{\prime}$ temos que $\sum_{n=1}^{\infty}\left|f\left(x_{n}\right)\right|^{p}<\infty$. $O$ espaço de todas as seqüências de um espaço de Banach $E$ que são $l_{p}-$ fracas será denotado por $l_{p}^{\omega}(E)$. 
Para um número real $p>1$, denotamos por $q$ o conjugado de $p$.

Proposição 3.2.2. Seja $F$ um espaço de Banach. Então as seguintes afirmações são verdadeiras.

(a) $S e\left(y_{n}\right)_{n} \in l_{p}^{\omega}(F)$, para $p \geq 1$ então $\left(y_{n}\right)_{n} \in l_{r}^{\omega}(F)$ para todo $r \geq p$.

(b) Seja $\left(e_{n}\right)_{n}$ a base canônica de $l_{p}$, para $1<p<\infty$ então $\left(e_{n}\right)_{n} \in l_{q}^{\omega}\left(l_{p}\right)$.

(c) Seja $\left(e_{n}\right)_{n}$ a base canônica de $c_{0}$, então $\left(e_{n}\right)_{n} \in l_{1}^{\omega}\left(c_{0}\right)$.

Demonstração: (a) Se $\left(y_{n}\right)_{n} \in l_{p}^{\omega}(F)$, então para todo $f \in F^{\prime}$ temos que $\sum\left|f\left(y_{n}\right)\right|^{p}<\infty$, ou seja, $\left(f\left(y_{n}\right)\right)_{n} \in \ell_{p}$. Como $\ell_{p} \subseteq \ell_{r}$ para $p \leq r$ segue que $\left(f\left(y_{n}\right)\right) \in \ell_{r}$ para $p \leq r$ e para todo $f \in F^{\prime}$, isto é, $\left(y_{n}\right)_{n} \in l_{r}^{\omega}(F)$ para $p \leq r$.

(b) Seja $\left(e_{n}\right)_{n}$ base canônica de $\ell_{p}$ para $1<p<\infty$. Como $\left(\ell_{p}\right)^{\prime} \cong \ell_{q}$, para cada $\phi \in\left(\ell_{p}\right)^{\prime}$, existe $\left(a_{j}\right)_{j} \in \ell_{q}$ tal que $\phi(x)=\sum a_{j} x_{j}$ para cada $x=\left(x_{j}\right)_{j} \in l_{p}$. Assim, para cada $\phi \in\left(\ell_{p}\right)^{\prime}$ temos que $\sum\left|\phi\left(e_{n}\right)\right|^{q}=\sum\left|a_{n}\right|^{q}<\infty$. Portanto, $\left(e_{n}\right)_{n} \in l_{q}^{\omega}\left(\ell_{p}\right)$.

(c) Seja $\left(e_{n}\right)_{n}$ base canônica de $c_{0}$. Como $\left(c_{0}\right)^{\prime} \cong \ell_{1}$, para cada $\phi \in\left(c_{0}\right)^{\prime}$, existe $\left(a_{j}\right)_{j} \in l_{1}$ tal que $\phi(x)=\sum a_{j} x_{j}$ para cada $x=\left(x_{j}\right)_{j} \in c_{0}$. Assim, para cada $\phi \in\left(c_{0}\right)^{\prime}$ temos que $\sum\left|\phi\left(e_{n}\right)\right|=\sum\left|a_{n}\right|<\infty$. Portanto, $\left(e_{n}\right)_{n} \in l_{1}^{\omega}\left(c_{0}\right)$.

$\mathrm{Na}$ demonstração da proposição 3.2 .5 utilizaremos fortemente a proposição que apresentamos a seguir. Nesta última, para demonstrar que $(\mathrm{a}) \Rightarrow(\mathrm{b})$ utilizamos o fato que se $\left(y_{n}\right)_{n}$ é uma seqüência em $l_{p}^{\omega}(F)$, com $F$ um espaço de Banach e $1<p<\infty$, então a série $\sum a_{n} y_{n}$ converge incondicionalmente em $F$, para toda seqüência $\left(a_{n}\right)_{n} \in \ell_{q}$. A demonstração deste resultado é uma modificação da prova de um resultado de Diestel (ver [5], pág. 44, teorema 6).

Proposição 3.2.3. Sejam $\left(y_{n}\right)_{n}$ uma seqüência em um espaço de Banach $F$ e $1<p<\infty$, então as seguintes afirmações são equivalentes:

(a) A seqüência $\left(y_{n}\right)_{n} \in l_{p}^{\omega}(F)$.

(b) Existe um operador $T \in L\left(\ell_{q}, F\right)$ tal que $T\left(e_{n}\right)_{n}=y_{n}$, com $\left(e_{n}\right)_{n}$ base canônica de $\ell_{q}$

Demonstração: (a) implica (b). Suponhamos que $\left(y_{n}\right)_{n} \in l_{p}^{\omega}(F)$, ou seja, $\sum\left|f\left(y_{n}\right)\right|^{p}<\infty$ para cada $f \in F^{\prime}$. 
Primeiro, definamos o operador linear $S: F^{\prime} \rightarrow \ell_{p}$ por $S(f)=\left(f\left(y_{n}\right)\right)_{n}$ para cada $f \in F^{\prime}$. O operador $S$ está bem definido pois $\left(y_{n}\right)_{n} \in l_{p}^{\omega}(F)$. Usaremos o Teorema do Gráfico Fechado, teorema 1.1.10, para mostrar que $S$ é contínuo.

Sejam $G_{S}=\left\{(f, S f): f \in F^{\prime}\right\} \subset F^{\prime} \times \ell_{p}$, o gráfico de $S$, e $(f, g) \in \overline{G_{S}}$. Então existe $\left(f_{n}, g_{n}\right) \in G_{S}$ tal que $\left(f_{n}, g_{n}\right) \rightarrow(f, g)$. Logo, $f_{n} \rightarrow f$ em $F^{\prime}$ e $S\left(f_{n}\right)=g_{n} \rightarrow g$ em $\ell_{p}$. Vamos mostrar que $g=S(f)$.

Observemos que como $S\left(f_{n}\right)=g_{n} \rightarrow g$ em $\ell_{p}$, a seqüência $\left(S f_{n}\right)_{n}$ é de Cauchy em $\ell_{p}$, logo, dado $\epsilon>0$ existe um número natural $j_{0}$ tal que $\left\|S f_{i}-S f_{j}\right\|_{p}<\epsilon$ para todos $i, j>j_{0}$. Isto é, $\sum_{n=1}^{\infty}\left|f_{i}\left(y_{n}\right)-f_{j}\left(y_{n}\right)\right|^{p}<\epsilon^{p}$ para todos $i, j>j_{0}$.

Em particular, $\sum_{n=1}^{N}\left|f_{i}\left(y_{n}\right)-f_{j}\left(y_{n}\right)\right|^{p}<\epsilon^{p}$ para todos $i, j>j_{0}$ e para todo $N \in \mathbb{N}$. Fazendo $j \rightarrow \infty$, temos que $\sum_{n=1}^{N}\left|f_{i}\left(y_{n}\right)-f\left(y_{n}\right)\right|^{p}<\epsilon^{p}$. E como isto vale para todo $N \in \mathbb{N}$, temos que $\left\|S f_{i}-S f\right\|_{p}^{p}=\sum_{n=1}^{\infty}\left|f_{i}\left(y_{n}\right)-f\left(y_{n}\right)\right|^{p} \leq \epsilon^{p}$ para todos $i>j_{0}$. Logo $S f_{n} \rightarrow S f$ em $\ell_{p}$. Como $S\left(f_{n}\right) \rightarrow g$, segue que $S(f)=g$. Portanto, segue do teorema do gráfico fechado que $S$ é contínuo.

No que segue, vamos mostrar que $\sum a_{n} y_{n}$ é incondicionalmente convergente em $F$ e a partir daí construir $T$. Sejam $\left(a_{n}\right)_{n} \in \ell_{q}$ e $f \in F^{\prime}$ tal que $\|f\| \leq 1$. Para cada $i, j$ naturais, temos que:

$$
\begin{gathered}
\left|f\left(\sum_{n=i}^{j} a_{n} y_{n}\right)\right|=\left|\sum_{n=i}^{j} a_{n} f\left(y_{n}\right)\right| \leq\left\|\left(0,0, \ldots, a_{i}, \ldots, a_{j}, 0,0, \ldots\right)\right\|_{q}\|S(f)\| \leq \\
\leq\left(\sum_{n=i}^{j}\left|a_{n}\right|^{q}\right)^{1 / q}\|S\|\|f\| \leq\left(\sum_{n=i}^{j}\left|a_{n}\right|^{q}\right)^{1 / q}\|S\| .
\end{gathered}
$$

Então,

$$
\sup _{\|f\| \leq 1}\left|f\left(\sum_{n=i}^{j} a_{n} y_{n}\right)\right| \leq\left(\sum_{n=i}^{j}\left|a_{n}\right|^{q}\right)^{1 / q}\|S\|
$$

Como, pelo teorema 1.1.3,

$$
\begin{gathered}
\sup _{\|f\| \leq 1}\left|f\left(\sum_{n=i}^{j} a_{n} y_{n}\right)\right|=\left\|\sum_{n=i}^{j} a_{n} y_{n}\right\|, \text { temos que } \\
\left\|\sum_{n=i}^{j} a_{n} y_{n}\right\| \leq\left(\sum_{n=i}^{j}\left|a_{n}\right|^{q}\right)^{1 / q}\|S\| \text { para todos } i, j \text { naturais. }
\end{gathered}
$$


Como $\left(a_{n}\right)_{n} \in \ell_{q},\left(\sum_{n=i}^{j}\left|a_{n}\right|^{q}\right)^{1 / q} \rightarrow 0$ quando $j \rightarrow \infty$. Logo, $\left\|\sum_{n=i}^{j} a_{n} y_{n}\right\| \rightarrow 0$ quando $j \rightarrow \infty$. Portanto a série $\sum a_{n} y_{n}$ é Cauchy somável, e como $F$ é um espaço de Banach, pela proposição 1.3.2, a série é incondicionalmente convergente em $F$.

Em seguida, definamos o operador $T: \ell_{q} \rightarrow F$ por $T(a)=\sum a_{n} y_{n}$ para cada $a=$ $\left(a_{n}\right)_{n} \in \ell_{q}$. O operador $T$ está bem definido, é linear e $T\left(e_{n}\right)=y_{n}$ para cada $n \in \mathbb{I N}$. Vamos mostrar que $T$ é contínuo. Para cada $a=\left(a_{n}\right)_{n} \in \ell_{q}$, usando 3.1, segue que

$$
\|T(a)\|=\left\|\sum_{n=1}^{\infty} a_{n} y_{n}\right\| \leq\|a\|_{q}\|S\| .
$$

Logo, $\|T\| \leq\|S\|$ e portanto $T$ é contínuo.

(b) implica (a). Suponha $T \in L\left(\ell_{q}, F\right)$ e $T\left(e_{n}\right)=y_{n}$ para cada $n$, com $\left(e_{n}\right)_{n}$ base canônica de $\ell_{q}$. Seja $f \in F^{\prime}$ qualquer. Então

$$
\sum\left|f\left(y_{n}\right)\right|^{p}=\sum\left|f\left(T\left(e_{n}\right)\right)\right|^{p}=\sum\left|(f \circ T)\left(e_{n}\right)\right|^{p}<\infty
$$

pois $f \circ T \in\left(\ell_{q}\right)^{\prime}$ e pela proposição 3.2.2(b), $\left(e_{n}\right)_{n} \in l_{p}^{\omega}\left(\ell_{q}\right)$. Portanto $\left(y_{n}\right)_{n} \in \ell_{p}^{\omega}(F)$.

Substituindo-se $l_{p}^{\omega}(F)$ por $l_{1}^{\omega}(F)$ e $\ell_{q}$ por $c_{0}$ na proposição 3.2 .3 obtemos o seguinte resultado que também será utilizado na demonstração da proposição 3.2.5.

Proposição 3.2.4. Seja $\left(y_{n}\right)_{n}$ uma seqüência em um espaço de Banach $F$ então as seguintes afirmações são equivalentes.

(a) A seqüência $\left(y_{n}\right)_{n} \in l_{1}^{\omega}(F)$.

(b) Existe um operador $T \in L\left(c_{0}, F\right)$ tal que $T\left(e_{n}\right)_{n}=y_{n}$, com $\left(e_{n}\right)_{n}$ base canônica de $c_{0}$.

Demonstração: (a) implica (b). A idéia da demonstração desta implicação é análoga a demonstração da implicação $(\mathrm{a}) \Rightarrow(\mathrm{b})$ da proposição anterior com as adequadas mudanças nas normas.

(b) implica (a). Suponha $T \in L\left(c_{0}, F\right)$ e $T\left(e_{n}\right)=y_{n}$ para cada $n$ natural, com $\left(e_{n}\right)_{n}$ base canônica de $c_{0}$. Seja $f \in F^{\prime}$ qualquer. Então

$$
\sum\left|f\left(y_{n}\right)\right|=\sum\left|f\left(T\left(e_{n}\right)\right)\right|=\sum\left|(f \circ T)\left(e_{n}\right)\right|<\infty
$$


pois $f \circ T \in\left(c_{0}\right)^{\prime}$ e pela observação $3.2 .2(\mathrm{c})\left(e_{n}\right)_{n} \in l_{1}^{\omega}\left(c_{0}\right)$. Portanto $\left(y_{n}\right)_{n} \in l_{1}^{\omega}(F)$.

Proposição 3.2.5. Sejam F um espaço de Banach $e 1<p<\infty$. As seguintes afirmações são válidas:

(a) $L\left(\ell_{p}, F\right)=K\left(\ell_{p}, F\right)$ se, e somente se, toda seqüência em $l_{q}^{\omega}(F)$ converge a zero em $F$.

(b) $L\left(c_{0}, F\right)=K\left(c_{0}, F\right)$ se, e somente se, toda seqüência em $l_{1}^{\omega}(F)$ converge a zero em $F$.

(c) $L\left(\ell_{1}, F\right)=K\left(\ell_{1}, F\right)$ se, e somente se, $F$ tem dimensão finita.

Demonstração: $(\mathrm{a})(\Rightarrow)$ Suponhamos que $L\left(\ell_{p}, F\right)=K\left(\ell_{p}, F\right)$. Seja $\left(y_{n}\right)$ uma sequiência qualquer em $l_{q}^{\omega}(F)$. Pela proposição $3.2 .3(\mathrm{a})(\Rightarrow)$ (b), existe um operador linear contínuo $T \in L\left(\ell_{p}, F\right)$ tal que $T\left(e_{n}\right)=y_{n}$, para todo $n \in \mathbb{N}$, com $\left(e_{n}\right)_{n}$ a base canônica de $\ell_{p}$.

Vamos proceder a demonstração por contradição. Suponhamos que $\left(y_{n}\right)_{n}$ não converge a zero em $F$. Então existe uma subseqüência $\left(y_{n k}\right)_{k}$ tal que $\left\|y_{n k}\right\|>\epsilon$ para algum $\epsilon>0$ e para todo $k \in \mathbb{N}$.

Como $\left(e_{n k}\right)_{k}$ é uma seqüência limitada em $\ell_{p}$ e $T$ é um operador compacto, segue que a seqüência $\left(T e_{n k}\right)_{k}$, isto é, $\left(y_{n k}\right)_{k}$ tem uma subseqüência convergente, $\left(y_{n k l}\right)_{l}$. Suponhamos que $y_{n k l} \rightarrow y \in F$. Então $\left(y_{n k l}\right)_{l}$ converge fracamente a $y$.

Como $\left(y_{n}\right)_{n} \in l_{q}^{\omega}(F),\left(y_{n}\right)_{n}$ converge fracamente a zero. conseqüentemente $\left(y_{n k l}\right)_{l}$ também converge fracamente a zero. Logo $y=0$. Portanto $y_{n k l} \rightarrow 0$, uma contradição.

$(\Leftarrow)$ Reciprocamente, suponhamos que toda seqüência em $l_{q}^{\omega}(F)$ converge a zero. Sejam $\left(x_{n}\right)_{n}$ uma seqüência limitada em $\ell_{p}$ e $T \in L\left(\ell_{p}, F\right)$ um operador qualquer. Vamos mostrar que $\left(T x_{n}\right)_{n}$ tem uma subseqüência convergente em $F$.

Como $\ell_{p}$ é reflexivo, pelo lema $1.2 .15,\left(x_{n}\right)_{n}$ tem uma subseqüência fracamente convergente. Sem perda de generalidade, podemos assumir que $\left(x_{n}\right)_{n}$ é fracamente convergente. Suponhamos que $x_{n} \stackrel{\omega}{\rightarrow} x \in \ell_{p}$.

Se $\lim _{n \rightarrow \infty}\left\|x_{n}-x\right\|=0$, pela continuidade de $T$ teríamos o resultado. Vamos agora verificar que se $\lim _{n \rightarrow \infty}\left\|x_{n}-x\right\|>0$ então $T\left(x_{n}\right)_{n}$ admite uma subsequiência convergente. Segue do Princípio de Seleção de Bessaga-Pelczynski (teorema 1.3.29) que $\left(x_{n}-x\right)_{n}$ admite uma subseqüência que é uma seqüência básica equivalente a uma seqüência de blocos da 
base canônica de $\ell_{p}$. Sem perda de generalidade, podemos assumir que $\left(x_{n}-x\right)_{n}$ é tal sequiência. Pelo teorema 1.3.28, $\left(x_{n}-x\right)$ é equivalente à base canônica de $\ell_{p}$.

Como a base canônica de $\ell_{p}$ está em $l_{q}^{\omega}\left(\ell_{p}\right)$, segue que $\left(x_{n}-x\right) \in l_{q}^{\omega}\left(\ell_{p}\right)$. Logo $\left(T\left(x_{n}-x\right)\right) \in l_{q}^{\omega}(F)$. Conseqüentemente, por hipótese, $\left(T\left(x_{n}-x\right)\right)_{n}$ converge a zero em $F$. Assim, para toda sequêencia $\left(x_{n}\right)_{n}$ limitada em $\ell_{p},\left(T x_{n}\right)_{n}$ admite uma subseqüência convergente em $F$ e $T \in K\left(\ell_{p}, F\right)$.

(b) $(\Rightarrow)$ Suponhamos que $L\left(c_{0}, F\right)=K\left(c_{0}, F\right)$. Seja $\left(y_{n}\right)$ uma seqüência qualquer em $l_{1}^{\omega}(F)$. Pela proposição $3.2 .4(\mathrm{a})(\Rightarrow)(\mathrm{b})$, existe um operador linear contínuo $T \in L\left(c_{0}, F\right)$ tal que $T\left(e_{n}\right)=y_{n}$, para todo $n \in \mathbb{N}, \operatorname{com}\left(e_{n}\right)_{n}$ a base canônica de $c_{0}$.

Procedendo por contradição, vamos supor que $\left(y_{n}\right)_{n}$ não converge a zero. Então existe uma subseqüência $\left(y_{n k}\right)_{k}$ tal que $\left\|y_{n k}\right\|>\epsilon$ para algum $\epsilon>0$ e para todo $k \in \mathbb{N}$.

Como $\left(e_{n k}\right)_{k}$ é uma seqüência limitada em $\ell_{p}$ e $T$ é um operador compacto, a seqüência $\left(T e_{n k}\right)_{k}$, isto é, $\left(y_{n k}\right)_{k}$ tem uma subseqüência convergente, $\left(y_{n k l}\right)_{l}$ em $F$. Suponhamos que $y_{n k l} \rightarrow y \in F$. Então $y_{n k l}$ converge fracamente a $y$.

Como $\left(y_{n}\right)_{n} \in l_{1}^{\omega}(F),\left(y_{n}\right)_{n}$ converge fracamente a zero. Então $y_{n k l}$ também converge fracamente a zero. Logo $y=0$. Portanto $y_{n k l} \rightarrow 0$, uma contradição.

$(\Leftarrow)$ Para a recíproca, suponhamos que toda seqüência em $l_{1}^{\omega}(F)$ converge a zero em $F$. Notemos que a base canônica de $c_{0}$ pertence a $l_{1}^{\omega}\left(c_{0}\right)$ e que não converge a zero. Logo $c_{0} \nLeftarrow F$. Então pela proposição 3.1.1, $L\left(c_{0}, F\right)=K\left(c_{0}, F\right)$.

(c) $(\Rightarrow)$ Suponhamos por contradição que $F$ tem dimensão infinita. Então podemos escolher $y_{1}$ de norma 1 em $F$. Seja $Y_{1}$ o subespaço de $F$ gerado por $y_{1}$. Pelo Lema de Riesz, lema 1.1.34, existe $y_{2} \in S_{F}$ tal que $\left\|y_{2}-y_{1}\right\|>1 / 2$. Seja $Y_{2}$ o subespaço de $F$ gerado por $y_{1}$ e $y_{2}$. Pelo Lema de Riesz, lema 1.1.34, existe $y_{3} \in S_{F}$ tal que $\left\|y_{3}-y_{1}\right\|>1 / 2$ e $\left\|y_{3}-y_{2}\right\|>1 / 2$.

Desta maneira, por indução, construímos uma seqüência $\left(y_{n}\right)_{n} \in F$ de norma 1 e tal que $\left\|y_{m}-y_{n}\right\|>1 / 2$ para $m \neq n$. Claramente, $\left(y_{n}\right)_{n}$ não admite subseqüência convergente.

Vamos agora construir um operador contínuo e não compacto de $\ell_{1}$ em $F$. 
Seja $T: \ell_{1} \rightarrow F$ um operador linear dado por $T(a)=\sum a_{n} y_{n}$ para cada $a=\left(a_{n}\right)_{n} \in \ell_{1}$. De $\left\|\sum a_{n} y_{n}\right\| \leq \sum\left\|a_{n} y_{n}\right\|=\sum\left|a_{n}\right|\left\|y_{n}\right\|=\sum\left|a_{n}\right|=\|a\|_{1}$, temos que o operador $T$ está bem definido e é contínuo.

Denotando por $\left(e_{n}\right)_{n}$ a base canônica de $\ell_{1}$, para cada $n \in \mathbb{N}$, temos que $T\left(e_{n}\right)=y_{n}$. Como $\left(y_{n}\right)_{n} \subset F$ é limitada e não admite subseqüência convergente, segue que $T \notin$ $K\left(\ell_{1}, F\right)$, contradizendo a hipótese inicial.

$(\Leftarrow)$ Se $F$ tem dimensão finita então é imediato que $L\left(\ell_{1}, F\right)=K\left(\ell_{1}, F\right)$.

Corolário 3.2.6. Seja $F$ um espaço de Banach. Se $L\left(\ell_{p}, F\right)=K\left(\ell_{p}, F\right)$, para algum $p$, $1 \leq p<\infty$, então as seguintes afirmações são verdadeiras.

(a) $L\left(\ell_{r}, F\right)=K\left(\ell_{r}, F\right)$ para todo $r$ tal que $p \leq r<\infty$.

(b) $L\left(c_{0}, F\right)=K\left(c_{0}, F\right)$.

Demonstração: Se $p=1$, então pela proposição $3.2 .5(\mathrm{c}), F$ tem dimensão finita e conseqüentemente $L\left(\ell_{r}, F\right)=K\left(\ell_{r}, F\right)$ para todo $r$ tal que $p \leq r<\infty$ e $L\left(c_{0}, F\right)=$ $K\left(c_{0}, F\right)$.

(a) Se $1<p \leq r<\infty$ e $L\left(\ell_{p}, F\right)=K\left(\ell_{p}, F\right)$, pela proposição 3.2 .5 (a), toda seqüência em $l_{q}^{\omega}(F)$ converge a zero em $F$. Denotemos por $r^{\prime}$ o conjugado de $r$. Como $p \leq r$, temos que $r^{\prime} \leq q$.

Agora pela proposição $3.2 .2(\mathrm{a})$, temos que toda seqüência em $l_{r^{\prime}}^{\omega}(F)$ é uma seqüência em $l_{q}^{\omega}(F)$. Logo toda seqüência em $l_{r^{\prime}}^{\omega}(F)$ converge a zero em $F$. Agora novamente utilizando a proposição $3.2 .5(\mathrm{a})$, segue que $L\left(\ell_{r}, F\right)=K\left(\ell_{r}, F\right)$.

(b) Se $1<p \leq r<\infty$ e $L\left(\ell_{p}, F\right)=K\left(\ell_{p}, F\right)$, pela proposição 3.2 .5 (a), toda seqüência em $l_{q}^{\omega}(F)$ converge a zero em $F$, logo $F$ não possui cópia de $c_{0}$ (pois a base canônica de $c_{0}$ é uma seqüência em $l_{q}^{\omega}\left(c_{0}\right)$ que não converge a zero). Então, pela proposição 3.1.1, $L\left(c_{0}, F\right)=K\left(c_{0}, F\right)$.

Na seção 3, vamos precisar da seguinte proposição para demonstração do corolário 3.3.4.

Proposição 3.2.7. Seja F um espaço de Banach. Então as seguintes afirmações são equivalentes.

(a) Para cada espaço de Hilbert $H$ de dimensão infinita temos que $L(H, F)=K(H, F)$.

(b) Para algum espaço de Hilbert $H$ de dimensão infinita temos que $L(H, F)=$ 
$K(H, F)$.

(c) $L\left(\ell_{2}, F\right)=K\left(\ell_{2}, F\right)$.

(d) Toda seqüência em $l_{2}^{\omega}(F)$ converge a zero em $F$.

Demonstração: (a) implica (b) é imediato.

(b) implica (c) Suponhamos que $L\left(H_{1}, F\right)=K\left(H_{1}, F\right)$ para algum espaço de Hilbert $H_{1}$. Sejam $T: \ell_{2} \rightarrow F$ um operador contínuo e $\left(h_{n}\right)_{n}$ uma seqüência ortonormal qualquer em $H_{1}$. Então o subespaço de $H_{1}$ gerado por $\left(h_{n}\right)_{n}, G$, é separável. Logo, pela proposição 1.4.19, existe um isomorfismo $R: \ell_{2} \rightarrow G$.

Sejam $S=T \circ R^{-1}$. Então $S: G \rightarrow F$ é um operador contínuo. Como $T=S \circ R$, vamos mostrar que $S$ é um operador compacto, então teremos $T$ um operador compacto.

Para isso, seja $P: H_{1} \rightarrow G$ a projeção ortogonal de $H_{1}$ em $G$. Então $S \circ P: H_{1} \rightarrow F$ é um operador contínuo, e logo, por hipótese, compacto. Como $P$ é projeção de $H_{1}$ sobre $G$, cada subconjunto limitado de $G, B_{1}$, é imagem de um subconjunto limitado de $H_{1}$, $B_{2}$, e assim, $\overline{S\left(B_{1}\right)}=\overline{S\left(P\left(B_{2}\right)\right)}=\overline{(S \circ P)\left(B_{2}\right)}$ é um subconjunto compacto de $F$, pois $S \circ P$ é um operador compacto. Portanto $S$ também é compacto.

(c) implica (d) é a proposição 3.2.5 (a).

(d) implica (a) Sejam $H$ um espaço de Hilbert e $T \in L(H, F)$ quaisquer. Vamos mostrar que lim $\left\|T h_{n}\right\|=0$ para toda seqüência ortonormal $\left(h_{n}\right)_{n}$ de $H$. Assim, pelo teorema 1.4.23, teremos $T \in K(H, F)$.

Seja $\left(h_{n}\right)_{n}$ uma seqüência ortonormal qualquer em $H$. Então, pela proposição 1.4.21, $\left(h_{n}\right)_{n} \in l_{2}^{\omega}(H)$. Vamos mostrar que $\left(T h_{n}\right)_{n} \in l_{2}^{\omega}(F)$.

Para cada $\varphi \in F^{\prime}, \varphi \circ T \in H^{\prime}$, temos que $\sum\left|\varphi\left(T\left(h_{n}\right)\right)\right|^{2}=\sum\left|(\varphi \circ T)\left(h_{n}\right)\right|^{2}<\infty$. Então $\left(T h_{n}\right)_{n} \in l_{2}^{\omega}(F)$ e conseqüentemente, por hipótese, $\left(T h_{n}\right)_{n}$ converge a zero em $F$. 


\subsection{Caracterizações para Espaços Compactos Não Dispersos}

Nesta seção, $\Omega$ denotará um espaço de Hausdorff compacto não disperso. Nesse caso, apresentamos uma condição necessária para um espaço de Banach $F$ para que todo operador linear contínuo de $C(\Omega)$ em $F$ seja compacto. Especificamente, se cada operador linear contínuo de $C(\Omega)$ em $F$ é compacto então cada operador linear contínuo de $\ell_{p}$ em $F$ é compacto, para $p \geq 2$.

Como conseqüência temos que para $\Omega$ um espaço de Hausdorff compacto não disperso e $F$ um espaço de Banach para o qual cada operador linear contínuo de $C(\Omega)$ em $F$ é absolutamente 2-somante, então cada operador linear contínuo de $C(\Omega)$ em $F$ é compacto se, e somente se, cada operador linear contínuo de $\ell_{2}$ em $F$ é compacto.

Temos também como conseqüência que para $\Omega$ um espaço de Hausdorff compacto não disperso e $F$ um espaço de Banach, cada operador linear contínuo de $C(\Omega)$ em $F$ é compacto se, e somente se, cada operador linear contínuo de $\ell_{2}$ em $F$ é compacto e cada operador linear contínuo de $C(\Omega)$ em $F$ admite uma fatoração através de um subespaço fechado de $c_{0}$.

Proposição 3.3.1. Se F é um espaço de Banach com a propriedade de Schur, então $L(C(\Omega), F)=K(C(\Omega), F)$.

Demonstração: Seja $T \in L(C(\Omega), F)$ qualquer. Como $F$ tem a propriedade de Schur, $c_{0} \nrightarrow F$ (pois a base canônica de $c_{0}$ converge fracamente a zero, mas não converge em norma para zero). Então pelo teorema 1.4.27, $T$ é fracamente compacto. Novamente observando-se que $F$ tem a propriedade de Schur pela proposição 1.4 .25 , temos que $T$ é compacto.

Lema 3.3.2. Seja $F$ um espaço de Banach tal que $L\left(\ell_{2}, F\right) \neq K\left(\ell_{2}, F\right)$. Se $T: \ell_{2} \rightarrow$ $F$ é um operador linear contínuo e não é compacto, então existe uma seqüência básica equivalente à base canônica de $\ell_{2},\left(\mu_{n}\right)_{n} \in \ell_{2}$, tal que $\left(T \mu_{n}\right)_{n}$ não admite subseqüência convergente.

Demonstração: Como $T$ é não compacto, existe uma seqüência limitada $\left(x_{n}\right)_{n} \in \ell_{2}$ tal que $\left(T x_{n}\right)_{n}$ não admite subseqüência convergente. 
Como $\ell_{2}$ é reflexivo, pelo lema $1.2 .15,\left(x_{n}\right)_{n}$ admite uma subseqüência fracamente convergente. Sem perda de generalidade, podemos supor que $\left(x_{n}\right)_{n}$ é fracamente convergente, isto é, $x_{n} \stackrel{\omega}{\rightarrow} x$, para algum $x \in \ell_{2}$.

Então, pelo princípio de seleção de Bessaga e Pelczynski, teorema 1.3.29, existe uma subseqüência de $\left(x_{n}-x\right)_{n}$, que é uma seqüência básica equivalente a uma seqüência de blocos da base canônica de $\ell_{2}$. Sem perda de generalidade, podemos supor que $\left(x_{n}-x\right)_{n}$ é tal seqüência.

Logo, pelo teorema 1.3.28, $\left(\frac{x_{n}-x}{\left\|x_{n}-x\right\|}\right)_{n}$ é uma seqüência equivalente a base canônica de $\ell_{2}$. Além disso, como $\left(T x_{n}\right)_{n}$ não admite subseqüência convergente, temos que $\left(T\left(\frac{x_{n}-x}{\left\|x_{n}-x\right\|}\right)\right)_{n}$ não admite subseqüência convergente.

Teorema 3.3.3. Sejam $\Omega$ um espaço de Hausdorff, compacto, não disperso e $F$ um espaço de Banach. Se $L(C(\Omega), F)=K(C(\Omega), F)$ então $L\left(\ell_{p}, F\right)=K\left(\ell_{p}, F\right)$ para $p \geq 2$.

Demonstração: Pelo corolário 3.2.6, somente o caso $p=2$ precisa ser demonstrado.

Suponhamos por contradição que $L\left(\ell_{2}, F\right) \neq K\left(\ell_{2}, F\right)$. Então existe um operador não compacto $T \in L\left(\ell_{2}, F\right)$. Logo, pelo lema 3.3.2, existe uma seqüência básica $\left(\mu_{n}\right)_{n} \in \ell_{2}$ equivalente à base canônica de $\ell_{2}$, tal que $\left(T \mu_{n}\right)_{n}$ não admite subseqüência convergente em $F$.

Vamos agora definir um operador linear contínuo $\psi(T): C(\Omega) \rightarrow F$ que não é compacto.

Como $\Omega$ é um espaço de Hausdorff compacto não disperso, segue ${ }^{1}$ que existem um espaço de funções $G$ (que é um espaço de Hilbert) que contém uma seqüência básica de funções ortonormais $\left(r_{n}\right)_{n}$ equivalente à base canônica de $\ell_{2}$ e uma aplicação contínua $\Lambda: C(\Omega) \rightarrow G$ tal que para cada função $r_{n}$ e para cada número natural $k$, existe uma função $f_{n k} \in C(\Omega)$ tal que $\left\|f_{n k}\right\|=1$ e $\left\|\Lambda f_{n k}-r_{n}\right\|<1 / k$.

Seja $M$ o subespaço fechado de $G$ gerado pela seqüência $\left(r_{n}\right)_{n}$ e pelas seqüências $\left(\Lambda f_{n k}\right)_{k}$ para $n=1,2, \ldots$ Sejam $M_{1}$ o subespaço fechado de $M$ gerado pela seqüência $\left(r_{n}\right)_{n}$, e $M_{0}$ o complemento ortogonal de $M_{1}$ em $M$. Então $M$ é soma direta de $M_{1}$ e $M_{0}$.

\footnotetext{
${ }^{1} \mathrm{Na}$ realidade o espaço $G$ é o espaço $L_{2}(\mu)$ para uma medida apropriada. No entanto, por fugir ao escopo desta dissertação, não enunciaremos aqui estes resultados. Ver Diestel [7] pág.183 e Semadeni [24] pág. 338, teorema 19.7.6
} 
Seja $N$ o subespaço fechado de $l_{2}$ gerado por $\left(\mu_{n}\right)_{n}$.

Seja $P: G \rightarrow M$, a projeção ortogonal de $G$ sobre $M$. Como $\left(r_{n}\right)_{n}$ é equivalente à base canônica de $\ell_{2}$, segue que $\left(\mu_{n}\right)_{n}$ e $\left(r_{n}\right)_{n}$ são equivalentes, logo existe um isomorfismo $J^{\prime}$ de $M_{1}$ sobre $N$, tal que $J^{\prime}\left(r_{n}\right)=\mu_{n}$ para $n=1,2, \ldots$ O operador $J^{\prime}$ pode ser estendido para um operador contínuo $J: M_{1} \oplus M_{0} \rightarrow N$, com $J(x)=0$ para cada $x \in M_{0}$.

Seja $\psi(T)=\left.T\right|_{N} J P \Lambda$. Temos que $\psi(T)$ é um operador linear contínuo de $C(\Omega)$ em $F$. Afirmamos que $\psi(T)$ não é compacto.

Para isso é suficiente mostrar que $\left(T \mu_{n}\right)_{n} \subseteq \overline{\{\psi(T)(f): f \in C(\Omega) \text { e }\|f\|=1\}}$.

Para este fim, notemos que:

$$
\begin{aligned}
\left\|T \mu_{n}-\psi(T) f_{n k}\right\| & =\left\|T J P r_{n}-T J P \Lambda f_{n k}\right\| \leq\|T\|\|J\|\|P\|\left\|r_{n}-\Lambda f_{n k}\right\| \leq \\
\leq & \|T\|\|J\|\|P\| 1 / k \rightarrow 0 \text { quando } k \rightarrow \infty .
\end{aligned}
$$

Corolário 3.3.4. Seja $\Omega$ um espaço de Hausdorff compacto não disperso. Seja $F$ um espaço de Banach tal que $L(C(\Omega), F)=\Pi_{2}(C(\Omega), F)$. Então são as seguintes afirmações são equivalentes.

(a) $L(C(\Omega), F)=K(C(\Omega), F)$.

(b) $L\left(\ell_{2}, F\right)=K\left(\ell_{2}, F\right)$.

Demonstração: (a) implica (b) É o teorema 3.3.3.

(b) implica (a) Seja $T \in L(C(\Omega), F)$ qualquer. Então por hipótese temos que $T \in$ $\Pi_{2}(C(\Omega), F)$. Logo, pelo teorema de fatoração de Grothendieck-Pietsch (teorema 1.1.28), existe um espaço de Hilbert $H$ e operadores lineares $S \in L(C(\Omega), H)$ e $R \in L(H, F)$ tais que $T=R \circ S$.

Agora, por hipótese, $L\left(\ell_{2}, F\right)=K\left(\ell_{2}, F\right)$. Então pela proposição 3.2 .7 , temos que $L(H, F)=K(H, F)$. Logo $R$ é compacto e conseqüentemente $T=R \circ S \in K(C(\Omega), F)$.

Corolário 3.3.5. Sejam $\Omega$ um espaço de Hausdorff compacto não disperso e $F$ um espaço de Banach. Então as seguintes afirmações são equivalentes.

(a) $L(C(\Omega), F)=K(C(\Omega), F)$. 
(b) $L\left(\ell_{2}, F\right)=K\left(\ell_{2}, F\right)$ e cada $\left.T \in L(C \Omega), F\right)$ admite uma fatoração através de um subespaço fechado de $c_{0}$.

Demonstração: (a) implica (b) Se $L(C(\Omega), F)=K(C(\Omega), F)$, então pelo teorema 3.3.3, $L\left(\ell_{2}, F\right)=K\left(\ell_{2}, F\right)$ e, pelo teorema 1.4.12, cada $\left.T \in L(C \Omega), F\right)$ admite uma fatoração através de um subespaço fechado de $c_{0}$.

(b) implica (a) Seja $T \in L(C(\Omega), F)$ qualquer. Por hipótese, existem operadores $S \in L\left(C(\Omega), c_{0}\right)$ e $R \in L\left(c_{0}, F\right)$ tais que $T=R \circ S$.

Como por hipótese $L\left(\ell_{2}, F\right)=K\left(\ell_{2}, F\right)$, pela proposição 3.2.5 (a), toda seqüência em $l_{2}^{\omega}(F)$ converge a zero em $F$. Usando a proposição 3.2 .2 itens (c) e (a) segue que $c_{0} \leftrightarrow F$. Então, pela proposição $3.1 .1, L\left(c_{0}, F\right)=K\left(c_{0}, F\right)$. Assim $R$ é compacto e conseqüentemente $T \in K(C(\Omega), F)$.

\subsection{Fatoração}

Nesta seção $\Omega$ é um espaço de Hausdorff compacto (disperso ou não disperso). Se $E$ é um espaço de Banach, denotaremos por $\Phi_{E}(C(\Omega), C(\Omega))$ o conjunto dos operadores contínuos $T: C(\Omega) \rightarrow C(\Omega)$ que admitem uma fatoração através de $E$.

Aqui usaremos alguns dos teoremas anteriores para mostrar alguns resultados para o espaço $\Phi_{c_{0}}(C(\Omega), C(\Omega))$ de todos os operadores contínuos $T: C(\Omega) \rightarrow C(\Omega)$ que admitem uma fatoração através de $c_{0}$. Tal espaço contém o espaço de todos os operadores compactos de $C(\Omega)$ em $C(\Omega)$.

Proposição 3.4.1. Sejam $\Omega$ um conjunto infinito que é um espaço de Hausdorff compacto, e $X$ um subespaço fechado de $c_{0}$. Então as seguintes afirmações são válidas.

(a) $\Phi_{X}(C(\Omega), C(\Omega)) \subseteq \Phi_{c_{0}}(C(\Omega), C(\Omega))$.

(b) $K(C(\Omega), C(\Omega)) \subset \Phi_{c_{0}}(C(\Omega), C(\Omega))$.

Demonstração: (a) Seja $T \in \Phi_{X}\left(C(\Omega), C(\Omega)\right.$ ) qualquer e sejam $T_{1}: C(\Omega) \rightarrow X$ e $T_{2}: X \rightarrow C(\Omega)$ operadores contínuos tais que $T=T_{2} \circ T_{1}$. Como $X$ é um subespaço fechado de $c_{0}$, pelo teorema 1.1.29, $T_{2}$ estende-se para um operador linear contínuo $\hat{T}_{2}$ de $c_{0} \operatorname{em} C(\Omega)$. Assim $T=\hat{T}_{2} \circ T_{1} \in \Phi_{c_{0}}(C(\Omega), C(\Omega))$. 
(b) Seja $T \in K(C(\Omega), C(\Omega))$ qualquer. Pelo teorema 1.4.12, $T$ admite uma fatoração através de um subespaço fechado de $c_{0}$. Logo pelo item (a), $T \in \Phi_{c_{0}}(C(\Omega), C(\Omega))$. Portanto $K(C(\Omega), C(\Omega)) \subset \Phi_{c_{0}}(C(\Omega), C(\Omega))$.

Observamos que a inclusão dada no item b) é estrita, como mostra o exemplo a seguir.

Primeiro suponhamos $\Omega$ disperso. Como $\Omega$ é um conjunto infinito, pelo teorema 1.1.21, $C(\Omega)$ contém um subespaço complementado $M$ que é isométrico a $c_{0}$.

Sejam $P: C(\Omega) \rightarrow M$ a projeção contínua de $C(\Omega)$ sobre $M, J: M \rightarrow C(\Omega)$ a inclusão de $M$ em $C(\Omega)$ e $R: c_{0} \rightarrow M$ a isometria de $c_{0}$ sobre $M$. Então $J \circ P=J \circ R \circ R^{-1} \circ P$. $\mathrm{E}$ assim $J \circ P \in \Phi_{c_{0}}(C(\Omega), C(\Omega))$.

Para cada $n \in \mathbb{N}$, seja $z_{n}=R\left(e_{n}\right)$, com $\left(e_{n}\right)_{n}$ a base canônica de $c_{0}$. Então $\left(z_{n}\right)_{n}$ é uma seqüência limitada em $M$ que não admite subseqüência convergente. $\mathrm{E}(J \circ P)\left(z_{n}\right)=$ $J\left(z_{n}\right)=z_{n}$. Assim, $J \circ P$ não é compacto.

Suponhamos agora $\Omega$ não disperso. Como a base canônica $\left(e_{n}\right)_{n} \subset c_{0},\left(e_{n}\right)_{n} \in \ell_{2}^{\omega}\left(c_{0}\right)$, mas $e_{n} \nrightarrow 0$, segue da proposição 3.2.5 (a) que $L\left(\ell_{2}, c_{0}\right) \neq K\left(\ell_{2}, c_{0}\right)$. Assim, pelo teorema 3.3.3, $L\left(C(\Omega), c_{0}\right) \neq K\left(C(\Omega), c_{0}\right)$. Consideremos $T \in L\left(C(\Omega), c_{0}\right)$ e $T \notin$ $\left.K(C \Omega), c_{0}\right)$.

Agora observemos que como $\Omega$ não é disperso, segue do teorema 1.1.25 que existe um subespaço $M$ de $C(\Omega)$ isométrico a $c_{0}$. Seja $J: c_{0} \rightarrow M$ a isometria de $c_{0}$ sobre $M$. Claramente $J \circ T: C(\Omega) \rightarrow M$ é contínuo e admite uma fatoração através de $c_{0}$. Afirmamos que $J \circ T$ não é compacto. Vamos supor por absurdo que $J \circ T$ seja compacto, então, como $J$ é uma isometria de $c_{0}$ sobre $M$, temos que $J^{-1}: M \rightarrow c_{0}$ é contínuo, logo $J^{-1} \circ J \circ T=T$ é compacto, uma contradição. Assim $K(C(\Omega), C(\Omega)) \neq \Phi_{c_{0}}(C(\Omega), C(\Omega))$.

Teorema 3.4.2. Sejam $\Omega$ um espaço de Hausdorff compacto e $X$ um espaço de Banach separável. Então as seguintes afirmações são equivalentes.

(a) $\Phi_{X}(C(\Omega), C(\Omega)) \subseteq K(C(\Omega), C(\Omega))$.

(b) $\Phi_{X}(C(\Omega), C(\Omega)) \subset \Phi_{c_{0}}(C(\Omega), C(\Omega))$.

Demonstração: (a) implica (b) Suponhamos que $\Phi_{X}(C(\Omega), C(\Omega)) \subseteq K(C(\Omega), C(\Omega))$. Então pela proposição 3.4 .1 (b), $\Phi_{X}(C(\Omega), C(\Omega)) \subset \Phi_{c_{0}}(C(\Omega), C(\Omega))$. Observamos que $K(C(\Omega), C(\Omega)) \neq \Phi(C(\Omega), C(\Omega))$, assim $\Phi_{X}(C(\Omega), C(\Omega)) \neq \Phi_{c_{0}}(C(\Omega), C(\Omega))$.

(b) implica (a) Com as hipóteses dadas vamos ter que $c_{0} \hookrightarrow X$. De fato, se $c_{0} \hookrightarrow X$, 
então, como $X$ é separável, pelo teorema 1.1.26, existe uma projeção contínua $P$ de $X$ sobre $c_{0}$. Seja $J$ a inclusão de $c_{0}$ em $X$.

Agora consideremos $T \in \Phi_{c_{0}}(C(\Omega), C(\Omega))$ qualquer e $T=T_{2} \circ T_{1}$ uma fatoração de $T$ através de $c_{0}$. Então $T=T_{2} \circ P \circ J \circ T_{1}$. Logo $T \in \Phi_{X}(C(\Omega), C(\Omega))$. Assim, $\Phi_{c_{0}}(C(\Omega), C(\Omega)) \subset \Phi_{X}(C(\Omega), C(\Omega))$, contradizendo a hipótese. Assim temos que $c_{0} \uparrow X$.

Vamos agora mostrar que $\Phi_{X}(C(\Omega), C(\Omega)) \subseteq K(C(\Omega), C(\Omega))$. Para isto é suficiente mostrar que $L(C(\Omega), X)=K(C(\Omega), X)$, pois a composição de um operador compacto com um operador contínuo é um operador compacto.

Se $\Omega$ é disperso, pelo corolário 3.1 .2 (3) implica (1), temos que $L(C(\Omega), X)=$ $K(C(\Omega), X)$.

Se $\Omega$ não é disperso, então pelo teorema 1.1.25, existe uma isometria $J: X \rightarrow C(\Omega)$, de $X$ sobre um subespaço de $C(\Omega)$.

Seja $T \in L(C(\Omega), X)$ qualquer. Então $J \circ T \in \Phi_{X}(C(\Omega), C(\Omega))$. Logo, por hipótese, $J \circ T \in \Phi_{c_{0}}(C(\Omega), C(\Omega))$. Sejam $T_{1}: C(\Omega) \rightarrow c_{0}$ e $T_{2}: c_{0} \rightarrow J(X)$ operadores contínuos tais que $J \circ T=T_{2} \circ T_{1}$.

Observamos que como o operador $T_{2} \in L\left(c_{0}, J(X)\right), c_{0} \nrightarrow X, J$ é uma isometria de $X$ sobre $J(X)$, temos que $c_{0} \nrightarrow J(X)$. Então pelo corolário 3.1 .2 (3) implica (4), temos que $T_{2}$ é compacto. Logo $J \circ T=T_{2} \circ T_{1}$ é compacto. Como $J$ é uma isometria de $X$ sobre $J(X)$, temos que $J^{-1}: J(X) \rightarrow X$ é contínuo, logo $J^{-1} \circ J \circ T=T$ é compacto, e conseqüentemente $L(C(\Omega), X)=K(C \Omega), X)$. 


\section{Referências Bibliográficas}

[1] Aliprantis, C. D., Burkinshaw, O.: Positive Operators, Pure and Applied Mathematics Series, 119, Academic Press, New \& York London, 1985.

[2] Ansari, S. I.: On Banach spaces $Y$ for which $B(C(\Omega), Y)=K(C(\Omega), Y)$. Pacific J. Math., 169 (2)(1995), 201-218.

[3] Bessaga, C., Pelczynski, A.: On bases and uncondicional convergence of series in Banach spaces. Studia Math., 17 (1958), 151-164.

[4] Conway, J. B.: A Course in Functional Analysis, Springer-Verlag, New York, 1985.

[5] Diestel, J.: Sequences and Series in Banach Spaces, Springer-Verlag, New York, 1984.

[6] Diestel, J., Jarchow, H., Tonge, A.: Absolutely Summing Operators. Cambridge University Press, 1995.

[7] Diestel, J., Uhl, J. J.: Vector Measures. Mathematical Surveys, 15, Amer. Math. Soc., Providence, RI, 1977.

[8] Dunford, N., Schwartz, J. T.: Linear Operator, Part I. New York. 1958.

[9] Fabian, M.; Habala, P.; Hájek, P; Santalucía, V. M.; Pelant, J.; Zizler, V.: Function Analysis and Infinite-Dimensional Geometry. Canadian Mathematical Society: Springer-Verlag, 2001 .

[10] Goldberg, S.: Unbonded Linear Operators: Theory and Applications. New York: McGraw-Hill, 1966.

[11] Grothendieck, A.: Sur certains classes de suites dans les espaces de Banach, et le theoreme de Dvoretzky-Rogers. Boletim Soc. Mat. São Paulo, 8, (1956) 81-110. 
[12] Lacey, H. E.: The Isometric Theory of Classical Banach Spaces. Springer-Verlag, Berlin \& New York, 1974.

[13] Lindenstrauss, J., Pelczynski, A.: Contributions to the theory of classical Banach spaces. J. Funct. Anal. 8 (1971), 225-249.

[14] Lindenstrauss, J., Tzafriri, L.: Classical Banach Spaces I, Springer-Verlag, Berlin \& New York, 1977.

[15] McArthur, C. V., Retherford, J. R.: Some applications of an inequality in locally convex spaces. Trans. Amer. Math. Soc. 137, (1969) 115-123.

[16] Megginson, R. E.: An Introduction to Banach Spaces Theory. New York: Springer 1998.

[17] Pedersen, G. K.: Analysis Now. Graduate Texts in Mathematics, 118, SpringerVerlag, 1989.

[18] Pelczynski, A.: Projections in certain Banach spaces. Studia Math., 19 (1960), 209228.

[19] Pelczynski, A.: A theorem of Dunford-Pettis type for polynomial operators. Bul. Serie des sciences math., astro., et phys. XI (6) (1963), 379-386.

[20] Randtke, D. J.: Representation theorems for compact operator. Proc. Amer. Math. Soc. 37, (1973) 481-485.

[21] Randtke, D. J.: A compact operator characterization of $\ell_{1}$. Math. Ann. 208, (1974) $1-8$.

[22] Retherford, J. R.: Hilbert Space: Compact Operators and Trace Theorem, Cambridge University Press, 1993.

[23] Rosenthal, H. P.: On quasi-complemented subespaces of Banach spaces, with an appendix on compactnes of operators from $L^{p}(\mu)$ to $L^{r}(\nu)$. J. Funct. Anal.,4 (1969), $176-214$.

[24] Semadeni, Z.: Banach Spaces of Continuous Functions. Polish Scientific Publishers, Warsaw, 1971. 
[25] Singer, I.: Bases in Banach Spaces. New York: Springer-Verlag, 1970. 